\title{
Hirnmetabolische Veränderungen bei chronischem Rückenschmerz
}

\author{
Dissertation \\ zur Erlangung des akademischen Grades
}

doctor medicinae (Dr. med.)

vorgelegt dem Rat der Medizinischen Fakultät
der Friedrich-Schiller-Universität Jena

von Lisa Teresa Janetzki

geboren am 06.09.1990 in Köln 
Seite 2

\section{Gutachter}

1.

2.

3.

Tag der öffentlichen Verteidigung: 


\section{Inhaltsverzeichnis}

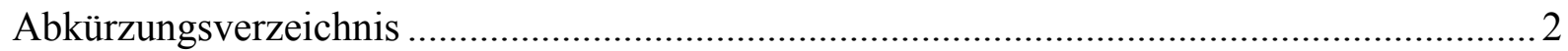

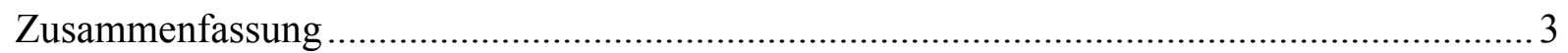

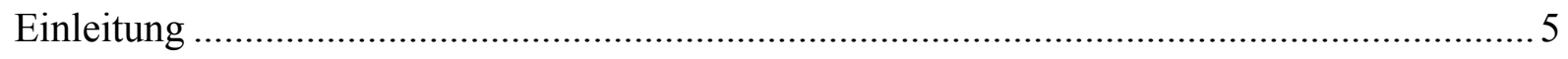

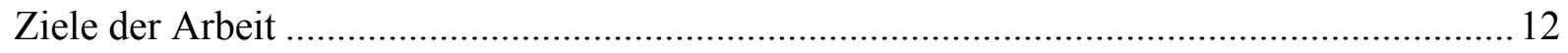

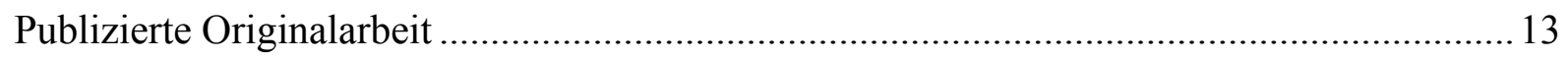

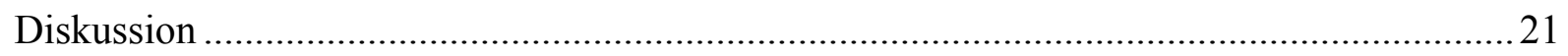

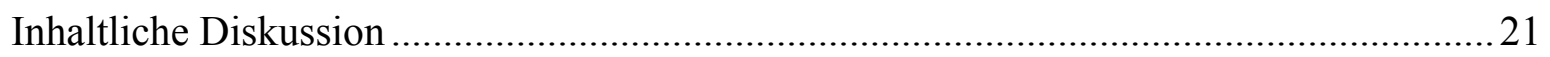

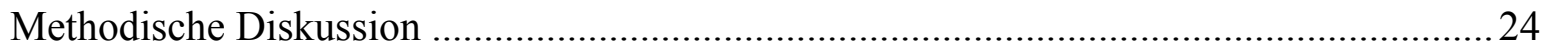

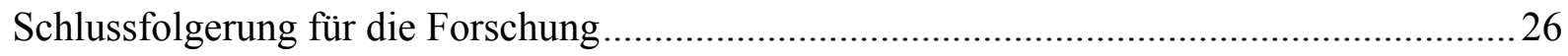

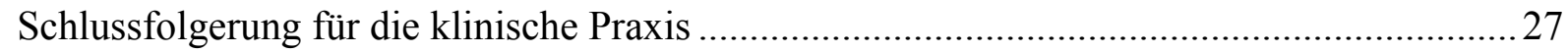

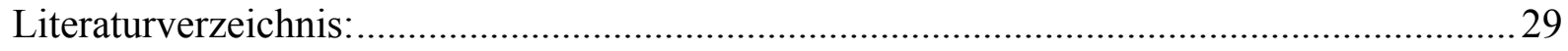

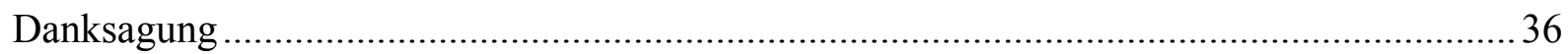

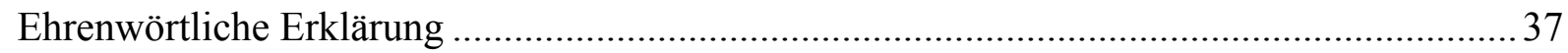

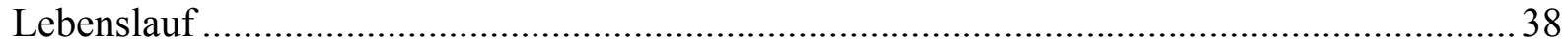

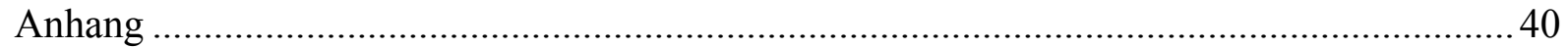

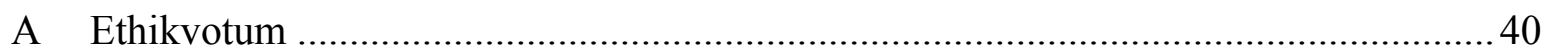

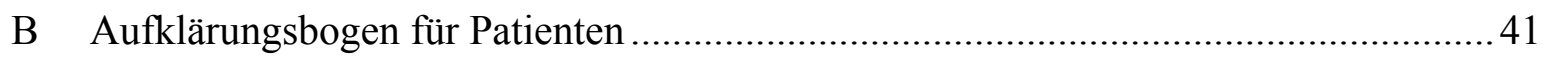

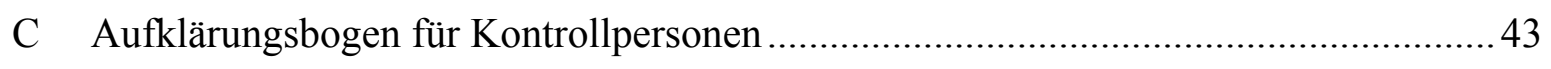

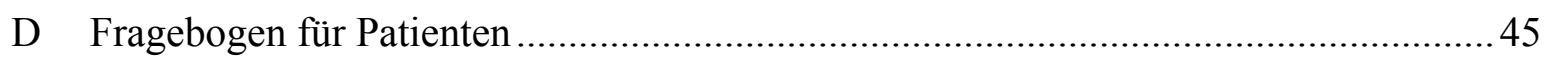

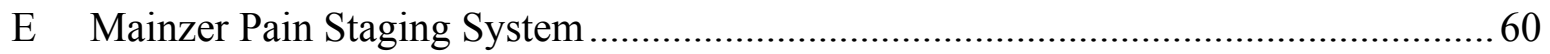




\section{Abkürzungsverzeichnis}

$\mathrm{aCC}$

AMPA

BÄK

$\mathrm{Cr}$

CURS

fMRT

GABA

Glx

${ }^{1} \mathrm{H}-\mathrm{MRS}$

HADS

IASP

ICD-10

IPMP

KIP

MD

MPSS

mGluR

NMDA

PET

RKI

SKID

SPECT

ZNS anteriorer cingulärer Kortex

Aminomethylphosphonsäure

Bundesärztekammer

Kreatin

Chronisch unspezifischer Rückenschmerz

Funktionelle Magnetresonanztomographie

$\gamma$-Aminobuttersäure

Summenwert aus Glutamin und Glutamat

Magnetresonanzspektroskopie

Hospital Anxiety and Depression Scale

International Association for the Study of Pain

International Statistical Classification of Diseases and

Related Health Problems, 10. Version

Institut für Psychosoziale Medizin und Psychotherapie

Kompetenzzentrum für Interdisziplinäre Prävention

Major Depression

Mainzer Stadienmodell der Schmerz-Chronifizierung

Metabolischer Glutamatrezeptor

$N$-Methyl-D-Aspartat

Positronen-Emissions-Tomographie

Robert-Koch-Institut

Strukturiertes klinisches Interview für DSM-IV

Single Photon Emission Computed Tomography

Zentrales Nervensystem 


\section{Zusammenfassung}

Chronische Rückenschmerzen gehören in Deutschland $\mathrm{zu}$ den häufigsten und kostenintensivsten Schmerzerkrankungen. Die Patienten weisen oft lange Krankengeschichten, eine dauerhaft eingeschränkte Lebensqualität und enorme psychische Belastungen auf. Auf Basis des biopsychosozialen Modells der Schmerzchronifizierung wird davon ausgegangen, dass neben klinischen und objektivierbaren Befunden, psychische Merkmale eine zentrale Rolle für die Entstehung, Aufrechterhaltung und Prognose von Schmerzsyndromen spielen. Außerdem besteht ein Zusammenhang zwischen dem Chronifizierungsstadium und dem Behandlungserfolg bei chronischen Schmerzpatienten.

Mittels funktioneller Bildgebung wurde festgestellt, dass chronische Schmerzen und die damit verbundenen psychologischen und funktionellen Beeinträchtigungen auch im Zusammenhang mit Änderungen des Neurotransmitterstoffwechsels in schmerzverarbeitenden Hirnregionen (z.B. anteriorer cingulärer Kortex (aCC), insulärer Kortex) stehen. Die Konzentrationen der im menschlichen Gehirn wichtigsten Neurotransmitter GABA ( $\gamma$-Aminobuttersäure) und Glutamat sind sowohl bei chronischen Schmerzen als auch bei psychischen Erkrankungen wie Depression und Angsterkrankungen verändert.

In dieser Studie wurde die Magnetresonanzspektroskopie $\left({ }^{1} \mathrm{H}-\mathrm{MRS}\right)$ genutzt, um Neurotransmitter im Gehirn in vivo $\mathrm{zu}$ quantifizieren und ein schmerzbegleitendes Ungleichgewicht zwischen erregend (glutamatergen) und hemmend (gabaergen) wirkenden Neurotransmittern bei Patienten mit chronischen unspezifischen Rückenschmerzen nachzuweisen sowie den Zusammenhang zu psychologischen und klinischen Befunden zu untersuchen.

Hierzu wurden bei 19 Patienten mit chronischen, unspezifischen Schmerzen (>3 Monate) im Rückenbereich sowie bei 19 nach Alter und Geschlecht parallelisierten gesunden Kontrollpersonen psychologische (Angst, Depressivität) und Schmerzmerkmale (Chronifizierungsstadium, Schmerzintensität, Schmerzdauer) mittels Fragebögen erfasst. Akute psychiatrische Erkrankungen wurden in einem standardisierten, klinischen Interview (SKID) ausgeschlossen. Die Neurotransmitter Glutamat und GABA sowie Glutamat/GABA Verhältnisse im aCC und im insulären Kortex wurden mittels ${ }^{1} \mathrm{H}-\mathrm{MRS}$ quantifiziert.

Es zeigte sich eine hohe Varianz der Glutamat/GABA Verhältnisse von Patienten und Kontrollpersonen in beiden gemessenen Hirnregionen. Ein signifikanter Unterschied zwischen den beiden Gruppen konnte nicht nachgewiesen werden. Das Merkmal „Angst“ war eine signifikante Einflussvariable auf die Konzentration von Glutamat in Insula und aCC. Das 
Merkmal „Depressivität“ wurde als Prädiktor für reduziertes GABA im aCC ermittelt. Außerdem zeigte sich innerhalb der Patientengruppe ein signifikanter Einfluss der Schmerzintensität auf GABA und Glutamat in der Insula. Des Weiteren wurde mit zunehmendem Chronifizierungsstadium eine Abnahme des Neurotransmitters Glutamat im aCC nachgewiesen. Dieser Zusammenhang wurde in der vorliegenden Studie erstmals beschrieben. In Übereinstimmung mit dem aktuellen Forschungsstand lässt sich ableiten, dass dem aCC und der Insula im Prozess der Schmerzchronifizierung unterschiedliche Rollen zukommen: Veränderungen der Botenstoffe standen im $\mathrm{aCC}$ in Zusammenhang mit psychischen Merkmalen, in der Insula mit der Schmerzintensität.

Die dargestellten Ergebnisse müssen unter Berücksichtigung folgender methodischer Limitationen interpretiert werden: mit ${ }^{1} \mathrm{H}-\mathrm{MRS}$ gemessene Neurotransmitterkonzentrationen repräsentieren Summenwerte der Messvolumina in den Hirnregionen, sodass kein Rückschluss auf die Herkunft der Metaboliten (Glutamat ist sowohl Metabolit im Energiestoffwechsel als auch Neurotransmitter) gezogen werden kann. Durch die Durchführung eines querschnittlichen Studiendesigns kann die Kausalität der gemessenen Zusammenhänge nicht bewertet werden. In Folgestudien sollten die nachgewiesen Effekte an größeren Stichproben und Kontrollgruppen überprüft werden.

In dieser Studie konnte nachgewiesen werden, dass sowohl klinische als auch psychische Merkmale von Patienten mit chronischen Rückenschmerzen im Zusammenhang mit Änderungen von Neurotransmittern in den schmerzverarbeitenden Hirnregionen aCC und Insula stehen. Die Vorgänge der zentralnervösen Schmerzverarbeitung und Chronifizierung sowie insbesondere den Bezug zu psychosozialen Merkmalen zu verstehen, stellt eine große Herausforderung für die aktuelle Schmerzforschung dar. In unserer Studie ist es gelungen eine Reihe von Einflussfaktoren zu identifizieren und die Bedeutung einer ausführlichen, individuellen Befunderhebung von sowohl psychischen als auch klinischen Parametern zu belegen. 


\section{Einleitung}

Epidemiologie

Rückenschmerzen zählen in Deutschland zu den wichtigsten Volkskrankheiten. Die Wahrscheinlichkeit während des Lebens an Rückenschmerzen zu erkranken wird auf 80-90\% geschätzt (Schmidt et al. 2007). Besonders häufig betroffen sind Frauen und Männer im erwerbstätigen Alter (30-65 Jahre). Erkrankungen des Muskel- und Skelettsystems zählten 2013 und 2014 laut Jahresberichten verschiedener Krankenkassen zu den Krankheiten mit den meisten Krankheitsfehltagen (21,5-26,5\%) und sind ein häufiger Grund für Frühberentungen (Techniker-Krankenkassen 2013, BKK 2013, DAK-Gesundheit 2014). Hierdurch entstehen hohe volkswirtschaftliche Kosten. Im Jahr 2008 beliefen sich die durch Rückenschmerzen entstandenen Krankheitskosten auf ca. 9 Milliarden Euro (Statistisches-Bundesamt 2010), wobei der größte Anteil (85\%) auf indirekte Kosten zurückzuführen ist, die durch den Verlust der Erwerbstätigkeit bei chronischen Schmerzen entstehen. Die individuellen Einschränkungen durch chronische Schmerzen sind enorm. Chronische Rückenschmerzpatienten sind in ihrer Lebensqualität stark eingeschränkt (Lame et al. 2005). Sie leiden unter emotionalen, körperlichen und geistigen Beeinträchtigungen, einer schlechteren Schlafqualität und schmerzbedingten Behinderungen im Alltag (Sezgin et al. 2014, Ling et al. 2007, Horng et al. 2005). Auf Grund der extrem hohen Lebenszeitprävalenz, den häufig langen Krankheitsverläufen und frustranen Therapieversuchen besteht die Herausforderung in erster Linie darin, Risikogruppen frühzeitig zu identifizieren und eine Chronifizierung der Schmerzen zu verhindern.

\section{Rückenschmerzen}

Nach Definition der Arbeitsgemeinschaft der Wissenschaftlichen Medizinischen Fachgesellschaften (AWMF) sind Rückenschmerzen (Kreuzschmerzen, Dorsopathie) Schmerzen im Bereich der Wirbelsäule vom Nacken- bis Steißbereich. Hinsichtlich des Verlaufes werden akute von chronischen Rückenschmerzen unterschieden. In 90\% der Fälle handelt es sich um akute Rückenschmerzen, die innerhalb von 12 Wochen, meist ohne Behandlung abklingen (Shekelle et al. 1995). Chronische Rückenschmerzen bestehen über einen Zeitraum von mindestens 12 Wochen, wobei die Intensität der Schmerzen in dieser Zeit variieren kann (IASP 1986).

Nur 10\% der Beschwerden im Rückenbereich lassen sich auf eine spezifische Ursache (z.B. Bandscheibenvorfall, Osteoporose, angeborene Fehlbildungen, entzündliche und traumatische Prozesse) zurückführen. In $90 \%$ der Fälle handelt es sich um unspezifische 
Rückenschmerzen. Hierbei besteht eine Diskrepanz zwischen klinischem Befund und subjektiv erlebter Beeinträchtigung der Patienten. Der Schmerz erfüllt nicht mehr die Funktion eines Warnsignals, sondern muss als eigenständiges Krankheitsbild verstanden werden (Pfingsten und Schops 2004).

\section{Das biopsychosoziale Modell}

Bei dem Prozess der Chronifizierung handelt es sich um ein komplexes biopsychosoziales Phänomen (Kröner-Herwig 2010). Zu den biologischen Faktoren werden neben orthopädischen oder neurologischen Grunderkrankungen auch funktionelle Störungen wie erhöhte Muskelspannung, Fehlbelastungen, Schonhaltungen, körperliche Inaktivität sowie neurophysiologische Dysfunktionen (Schmerzgedächtnis) gezählt. Die psychosozialen Faktoren umfassen Emotionen, Kognitionen und soziale Interaktionen. Als Prädiktoren für Chronifizierungswahrscheinlichkeit konnten Hasenbring et al. Depressivität, Copingverhalten, maladaptive schmerzbezogene Kognitionen, Zufriedenheit am Arbeitsplatz sowie allgemeinen Distress identifizieren (Hasenbring et al. 2001). Für einen Übergang von akuten zu chronischen Schmerzen weisen psychosoziale Faktoren den höheren prädiktiven Wert auf (Linton 2000). Sie sind als Risikofaktoren in der nationalen Versorgungsleitlinie für Kreuzschmerz der AWMF im „Flaggenmodell“ aufgeführt. Das Modell soll Ärzten der Primärversorgung die Diagnostik und prognostische Einschätzung von Kreuzschmerzen erleichtern. Stress-Empfindungen, Schmerzvermeidungsverhalten, Hilf- und Hoffnungslosigkeit, Katastrophisieren, passives Schmerzverhalten, „Somatisierungstendenz“ und negative Krankheitsvorstellungen sind „Gelbe Flaggen“, Warnhinweise für eine Chronifizierung. Zu den „Rote Flaggen“ zählen Tumore in der Vorgeschichte, fortschreitende neurologische Ausfälle, Fieber und unklarer Gewichtsverlust. Sie sind spezifische Warnhinweise für ernstzunehmende Ursachen der Rückenschmerzen und sollten von dem behandelten Arzt im Anamnesegespräch erfasst werden.

Die Chronifizierung von Rückenschmerzen verläuft nicht nach einem zeitlich festgelegten Schema, sondern ist von der Ursache und der individuellen Konstitution des Patienten abhängig. Rückenschmerzen können in unterschiedliche Stadien der Chronifizierung eingeteilt werden. Dies ist hilfreich, da ein Zusammenhang zwischen Behandlungserfolg einer Schmerztherapie und Chronifizierungsstadium besteht (Frettlöh et al. 2003). Ein etabliertes Modell ist das Mainzer Stadienmodell zur Schmerzchronifizierung nach Gerbershagen (MPSS: Mainz Pain Staging System). Es erlaubt eine Einteilung in drei 
Chronifizierungsstadien. Dabei werden zeitliche und räumliche Aspekte sowie Medikamenteneinnahmeverhalten und Patientenkarriere berücksichtigt (Gerbershagen, 1996).

\section{Psyche und chronischer Rückenschmerz}

Patienten mit chronischen Schmerzen weisen häufig eine hohe Anzahl von Komorbiditäten auf. Mit steigendem Chronifizierungsstadium nimmt auch die Anzahl der betroffenen Organsysteme zu (Buchner et al. 2007). Zu den häufigsten komorbiden Erkrankungen bei chronischem Rückenschmerz zählen Arthrose, Osteoporose und Depression (Buchner et al. 2007, Reme et al. 2011). Im Durchschnitt erfüllen 52\% aller Schmerzpatienten die Kriterien einer „Major Depression“ (MD). Im Vergleich: die Lebenszeitprävalenz für NichtSchmerzpatienten liegt in Deutschland bei 11,6\% (Busch et al. 2013). Ein noch größerer Anteil der Schmerzpatienten weist eine depressive Stimmung auf, ohne die Kriterien einer MD vollständig zu erfüllen (Bair et al. 2003). Für die Diagnostik und Behandlung chronischer Schmerzsyndrome ist eine Berücksichtigung dieser psychischen Merkmale essentiell. Patienten, die sowohl unter einer Depression als auch unter chronischen Schmerzen leiden, weisen längere und kompliziertere Krankheitsverläufe und geringere Therapieerfolge auf (Hasenbring 1998, McCracken und Turk 2002). Es kommt häufiger zu einer dauerhaften Einschränkung der Lebensqualität und einem Verlust der Selbstständigkeit. Wiederholte Behandlungsmisserfolge können in verstärkter Hilf- und Hoffnungslosigkeit resultieren, welche zu krankheitserhaltenden Überzeugungen führen und die Entstehung von Psychopathologien begünstigen (Gatchel et al. 2007). Es entsteht ein „Circulus vitiosus“, in dem biologische, psychologische und soziale Faktoren miteinander interagieren und den Schmerz aufrechterhalten. Bisher ungeklärt bleibt jedoch der kausale Zusammenhang zwischen Depressivität und chronischen Schmerzen. Schmerz kann das Symptom einer Depression sein, ebenso können chronische Schmerzen über verschiedene Mechanismen (sozialer Rückzug, Katastrophisieren, Hilflosigkeit) zu depressiven Stimmungen führen (Bair et al. 2003).

\section{Zentralnervöse Veränderungen bei chronischen Schmerzen}

Chronische Schmerzen hinterlassen Spuren im zentralen Nervensystem. Melzack und Wall prägten 2001 erstmals den Begriff der „Schmerzmatrix“ (Melzack 2001). Dabei handelt es sich um ein dynamisches Netzwerk von Hirnregionen, die an der Schmerzwahrnehmung und -kontrolle beteiligt sind: Thalamus, insulärer, präfrontaler, somatosensorischer und anteriorer cingulärer Kortex $(\mathrm{aCC})$. Sowohl auf struktureller als auch auf funktioneller Ebene finden 
sich bei Schmerzpatienten im Vergleich zu Kontrollpersonen Auffälligkeiten. Studien, die Kernspintomographie zur Darstellung morphologischer Veränderungen nutzen, zeigen bei Patienten mit chronischen Schmerzen eine spezifische Abnahme grauer Hirnsubstanz in Hirnregionen, die an der Schmerzverarbeitung beteiligt sind (Apkarian et al. 2004, Mao et al. 2013).

Bisher wurde neuronale Aktivität im Gehirn von Schmerzpatienten in erster Linie mit Techniken gemessen, welche sich den Zusammenhang zwischen lokalem Blutfluss sowie der lokalen Blutoxygenierung und Aktivierung in den Hirnregionen zunutze machen (SPECT, PET, fMRT). Ergebnisse dieser Studien deuten auf pathologische Aktivitätsmuster in Regionen der Schmerzmatrix bei chronischen Schmerzpatienten hin (Somborski und Bingel 2010). Häufig wird in diesem Zusammenhang von „zentraler Sensibilisierung“ gesprochen. Hierunter versteht man biochemische Veränderungen, welche durch eine erhöhte Membranerregbarkeit und synaptische Effizienz, sowie dem Verlust der physiologischen Hemmung zu dysfunktionalen Erregungsmustern und einer erhöhten Schmerzempfindlichkeit führen. Dieses sogenannte „Schmerzgedächtnis“ könnte den pathologischen Ursprung chronischer Schmerzsyndrome bilden (Latremoliere und Woolf 2009).

\section{Neurotransmitter}

Neurotransmitter sind biochemische Botenstoffe, welche Informationen (Reize) von Nervenzelle zu Nervenzelle übertragen. Der wichtigste erregende (exzitatorische) Neurotransmitter Glutamat wird unter anderem im Citratzyklus durch das Enzym Glutamatdehydrogenase aus $\alpha$-Ketoglutarat synthetisiert (Orrego und Villanueva 1993). Glutamat ist eine $\alpha$-Aminosäure, die im Gehirn in relativ hohen Konzentrationen $(<12 \mathrm{mM})$ vorliegt (Choi et al. 2006). Die Konzentration ist abhängig von der metabolischen Aktivität der Zellen. In sehr hohen Konzentrationen wirkt Glutamat über einen massiven Einstrom von Calzium-Ionen neurotoxisch. Es existieren 4 Subtypen von Glutamatrezeptoren im ZNS: drei ionotrope Rezeptoren (NMDA-, AMPA und Kainat-Rezeptor), die nach agonistisch wirkenden Substanzen benannt sind und ein metabotroper Rezeptor (mGluR). Glutamat ist an fast jeder Erregungsweiterleitung beteiligt. Im ZNS spielt Glutamat eine wichtige Rolle in sensorischen, emotionalen und kognitiven Prozessen und hat Einfluss auf die synaptische Plastizität (Sanacora et al. 2012). GABA ( $\gamma$-amino butyricacid, $\gamma$-Aminobuttersäure) ist der wichtigste hemmende (inhibitorische) Neurotransmitter und wird über das Enzym Glutamatdecarboxylase aus Glutamat synthetisiert. GABA wirkt über ionotrope $\left(\mathrm{GABA}_{\mathrm{A}}\right)$ und metabotrope $\left(\mathrm{GABA}_{\mathrm{B}}\right)$ Rezeptoren, die sich in prä- und postsynaptischen Membranen 
von Nervenzellen befinden. (Thomas Karov 2013). Während präsynaptische Rezeptoren über Rückkopplungshemmung die Ausschüttung des Transmitters regulieren, bewirken postsynaptische Rezeptoren über $\mathrm{Cl}^{-}$-Einstrom eine Hyperpolarisation der Zelle. Über diesen Mechanismus wird Erregungsleitung im ZNS gehemmt, verlangsamt und eingeschränkt. GABA ist an vielen physiologischen Prozessen beteiligt, unter anderem an Schlaf, Anästhesie, motorischer Kontrolle und Angst (Mohler 2006).

Mittels Magnetresonanzspektroskopie ( $\left.{ }^{1} \mathrm{H}-\mathrm{MRS}\right)$ ist es möglich Stoffwechselmetaboliten durch ihre charakteristische chemische Verschiebung (chemical shift) nicht-invasiv, am lebenden Patienten zu identifizieren und zu quantifizieren. Die Methode wird genutzt, um Neurotransmitter im Gehirn zu untersuchen und mit Erkrankungen zusammenhängende Veränderungen im Transmitterstoffwechsel zu beurteilen (Rae 2014). Glutamat wird als Summensignal des erregenden Neurotransmitters und des Zwischenproduktes Glutamin als Glx quantifiziert. Die ermittelten Intensitätswerte für GABA und Glx werden üblicherweise mit Kreatin (Gesamtkreatin, tCr) normiert, dessen Konzentration im Gehirn sowohl unter normalen physiologischen Bedingungen als auch bei den meisten Pathologien näherungsweise konstant bleibt und das deswegen gut als gewebeinterne Referenz geeignet ist. Daraus ergeben sich die Quotienten Glx/tCr und GABA/tCr als Stellvertreter für Erregung (Glutamat) und Hemmung (GABA) im Neurotransmitterstoffwechsel.

Bei chronischen Schmerzen liegt eine Veränderung des physiologischen Gleichgewichtes von Erregung und Hemmung in den schmerzverarbeitenden Hirnregionen vor. Mehrere Studien an Fibromyalgiepatienten konnten einen Anstieg der Glutamatkonzentrationen in den Hirnregionen insulärer Cortex, posteriorer cingulärer Cortex und Amygdala und niedrigere GABA-Konzentrationen im insulären Cortex nachweisen (Fayed et al. 2010, Foerster et al. 2012, Valdes et al. 2010). Die Anzahl der Studien, welche GABA in vivo im Gehirn von Schmerzpatienten nachweisen konnten, ist allerdings noch sehr gering. Auf Grund von Überlagerungen mit Spektren anderer Metaboliten und der geringen Gesamtkonzentration von GABA im ZNS (<2mmol/l) ist die Messung von GABA erschwert.

Besonders interessant ist die Betrachtung neurochemischer Veränderungen im Zusammenhang mit unspezifischen Rückenschmerzen. Eine der ersten Studien, die Neurotransmitter in aCC und Insula von 9 Rückenschmerzpatienten und 11 Kontrollpersonen untersuchte, konnte keine signifikante Änderung der Neurotransmitterkonzentrationen nachweisen (Grachev et al. 2000). Jedoch wurden interregionale Veränderungen der Schmerzmatrix, sowie ein Zusammenhang zwischen Angst (gemessen durch das State-Trait Anxiety Inventory) und Neurotransmitteränderungen nachgewiesen. Gussew et al. konnten 
2011 erniedrigte Glutamatkonzentrationen im aCC von Patienten mit chronisch unspezifischem Rückenschmerz (CURS) nachweisen. Zudem gibt es Hinweise auf eine positive Korrelation zwischen Glutamatkonzentration und Schmerzstärke im somatosensiblen Cortex (Sharma et al. 2011). Es sind jedoch weitere Studien an größeren Stichproben, sowie eine Berücksichtigung des Chronifizierungsstadiums notwendig, um diese Veränderungen näher zu belegen.

Glutamat- und GABA-Veränderungen spielen auch in der Pathophysiologie von psychischen Erkrankungen eine Rolle, jedoch sind die Ergebnisse auch hier auf Grund von messmethodischen Einschränkungen und heterogenen Stichproben nicht konsistent. Die bisher größte Evidenz existiert für den Zusammenhang von Depression und glutamaterger Dysfunktion. Einige Autoren (Sanacora et al. 2012) sprechen von einem Paradigmenwechsel, von der „monoamine hypothesis“ (Dunlop und Nemeroff 2007) hin zu einer „glutamate hypothesis“. Hierfür spricht eine beträchtliche Anzahl von Studien der letzten Jahre, die bei Patienten mit klinisch diagnostizierten Depressionen (ICD-10 F.32) niedrigere Glutamatkonzentrationen im cingulären und frontalen Cortex im Vergleich $\mathrm{zu}$ gesunden Kontrollpersonen nachwiesen (Auer et al. 2000, Hasler et al. 2007). Bei Patienten mit bipolarer Depression (ICD-10 F.31) wiesen mehrere Studien (Michael et al. 2003, Frye et al. 2007) erhöhte Glutamatkonzentrationen im anterioren cingulären und präfrontalen Cortex nach. Reduzierte GABA-Konzentrationen wurden im anterioren cingulären und occipitalen Cortex gemessen (Bhagwagar et al. 2008). Außerdem finden sich verschiedene Neurotransmitterveränderungen im aCC im Zusammenhang mit Angsterkrankungen und Phobien (Modi et al. 2014, Mahmutyazicioglu et al. 2005, Howells et al. 2014).

Auf Grund der Komplexität chronischer Schmerzsyndrome und neurochemischer Stoffwechselveränderungen, ist eine umfangreiche Diagnostik, welche sowohl psychische Symptome als auch Schmerzparameter umfasst, notwendig um das Zusammenspiel von Neurochemie, Schmerzen und psychosozialen Merkmalen zu erfassen.

Dieses Projekt gliedert sich in die langjährige Forschung zur Prävention und Behandlung chronischer Rückenschmerzen am Institut für Psychosoziale Medizin und Psychotherapie des Universitätsklinikums Jena sowie am Kompetenzzentrum für Interdisziplinäre Prävention der Friedrich-Schiller-Universität Jena ein. In zahlreichen Untersuchungen konnte die Bedeutung psychosozialer Faktoren für die Entstehung und Chronifizierung von Rückenbeschwerden sowie für die Inanspruchnahme und den Erfolg multimodaler Schmerztherapien nachgewiesen werden (Nötzel 2011, Borys et al. 2013, Borys et al. 2015, Tutzschke et al. 
2014). Parallel wurden am Institut für Diagnostische und Interventionelle Radiologie des Universitätsklinikums Jena seit 2010 mittels funktioneller Bildgebung die Auswirkung akuter und chronischer Schmerzen auf Botenstoffe in schmerzverarbeitenden Hirnregionen untersucht und die ${ }^{1} \mathrm{H}-\mathrm{MRS}$ zur Messung von Neurotransmitter-Konzentrationen im menschlichen Gehirn etabliert (Gussew et al. 2010, Gussew et al. 2011, Cleve et al. 2015). Eine Untersuchung der zentralen Botenstoffe unter Berücksichtigung psychosozialer Faktoren, welche für die Entstehung, Chronifizierung und den Therapieerfolg chronischer Schmerzen von essentieller Bedeutung sind, wurde in diesem Zusammenhang noch nicht durchgeführt. 


\section{Ziele der Arbeit}

Das Ziel der vorliegenden Arbeit war es, die zentralen Botenstoffe GABA und Glutamat im Gehirn chronischer Rückenschmerzpatienten unter Berücksichtigung klinischer und psychischen Parameter zu untersuchen.

Die Zusammenarbeit des Institutes für Psychosoziale Medizin und Psychotherapie (IPMP) des Universitätsklinikums Jena und des Institutes für Diagnostische und Interventionelle Radiologie des Universitätsklinikums Jena sowie der NONPain-Unit des Weimarer Sophienund Hufeland-Klinikums ist die erste dieser Art. Unterstützt wurde die Arbeit durch das Kompetenzzentrum für Interdisziplinäre Prävention (KIP) und gefördert durch die Berufsgenossenschaft Nahrungsmittel und Gastgewerbe. Im Rahmen des KIP wurden bereits zahlreiche Studien zur Prävention chronischer Schmerzen durchgeführt.

Besonderes Augenmerk wurde in dieser Studie auf die klinischen Merkmale Schmerzintensität, Schmerzdauer und das Chronifizierungsstadium sowie die psychischen Merkmale Depressivität und Angst gelegt. Der Zusammenhang von Depressivität und Angst bei Schmerzpatienten und neurochemischen Konzentrationen wurde bisher nur wenig untersucht, obwohl depressive Stimmung und erhöhte Ängstlichkeit häufige Befunde bei chronischen Schmerzpatienten sind. Allein eine Studie aus dem Jahr 2000 untersuchte Korrelationen von psychologischen Faktoren und GABA und Glutamat mit einer konventionellen MRS-Technik (Grachev et al. 2000). In Erweiterung wurden die Messungen dieser Studie an einem 3-T-MR-Scanner durchgeführt und neben der konventionellen ${ }^{1} \mathrm{H}$ MRS-Sequenz eine spezielle Spectral-editing-Sequenz verwendet, die eine zuverlässigere Quantifizierung der Metabolitensignale erlaubt.

Psychische Parameter sind nicht nur für die Entstehung, Aufrechterhaltung und die Prognose chronischer Schmerzsyndrome von essentieller Bedeutung, sondern weisen auch gemeinsame neurochemische Funktionswege (Veränderungen der Neurotransmitter GABA und Glutamat in aCC und Insula) auf. Ebenso fehlen bislang Studien, die den Zusammenhang zwischen Chronifizierungsstadium und neurochemischen Veränderungen untersuchen.

Ausgehend aus den offenen Forschungsfragen wurden die folgenden Hypothesen abgeleitet:

1. Im Vergleich zu gesunden Kontrollpersonen weisen Patienten erhöhte Glutamat- und verringerte GABA-Konzentrationen auf.

2. Depressivität und Angst stehen im Zusammenhang mit Glutamat- und GABAKonzentrationen.

3. Klinische Merkmale (Schmerzintensität, Schmerzdauer, Chronifizierungsstadium) stehen im Zusammenhang mit Glutamat- und GABA-Konzentrationen. 


\section{Publizierte Originalarbeit}

L. Janetzki, A. Gussew, R. Malessa, U. Habenicht, J.R. Reichenbach, B. Strauß, C. Borys. Hirnmetabolische Veränderungen bei chronischem Rückenschmerz. Schmerz: 1-7. Published ahead of print. DOI 10.1007/s00482-015-0082-5 
Schmerz

DOI 10.1007/s00482-015-0082-5

(c) Deutsche Schmerzgesellschaft e.V. Published by Springer-Verlag Berlin Heidelberg - all rights reserved 2015

CrossMark

L. Janetzki ${ }^{1}$ A. Gussew ${ }^{2} \cdot$ R. Malessa ${ }^{3} \cdot$ U. Habenicht ${ }^{3} \cdot$ J.R. Reichenbach ${ }^{2} \cdot$ B. Strauß ${ }^{1}$. C. Borys ${ }^{1}$

${ }^{1}$ Institut für Psychosoziale Medizin und Psychotherapie, Universitätsklinikum Jena,

Friedrich-Schiller-Universität, Jena, Deutschland

2 Medical Physics Group, Institut für Diagnostische und Interventionelle Radiologie,

Universitätsklinikum Jena, Friedrich-Schiller-Universität, Jena, Deutschland

${ }^{3}$ Klinik für Neurologie und Klinische Neurophysiologie, Sophien- und Hufeland-Klinikum,

Weimar, Deutschland

\section{Hirnmetabolische Veränderungen bei chronischem Rückenschmerz}

\section{Studie unter Berücksichtigung von klinischen und psychischen Parametern}

Chronische Rückenschmerzen gehören in Deutschland zu den häufigsten und mit den höchsten Kosten verbundenen Schmerzerkrankungen [19]. In 90\% der Fälle handelt es sich um unspezifische Rückenschmerzen mit einer erheblichen Diskrepanz zwischen subjektiven Beschwerden und objektivem Befund [14]. Damit verbunden sind Probleme bei der spezifischen Versorgung. Zur Charakterisierung der Schmerzpatienten und zur Einschätzung der Chronifizierungswahrscheinlichkeit steht eine Reihe von klinischen Parametern (Schmerzdauer, Schmerzintensität, Chronifizierungsstadium) und psychologischen Prädiktoren (Depressivität, Ängstlichkeit, Copingverhalten, schmerzbezogene Kognitionen) zur Verfügung [2, 12, 18].

Ergebnisse von Studien, die eine funktionelle Bildgebung [funktionelle Magnetresonanztomographie (fMRT), Elektroenzephalographie] zur Untersuchung neuronaler Aktivität einsetzen, deuten außerdem auf einen $\mathrm{Zu}$ sammenhang zwischen einer veränderten zentralen Schmerzverarbeitung und chronischen Schmerzen hin [13, 17, 22]. Als Folge dieser hirnfunktionellen Veränderungen wurde bei Schmerzpatienten darüber hinaus eine spezifische Abnahme grauer Hirnsubstanz in Hirnregionen nachgewiesen, die in die Schmerzverarbeitung involviert sind $[3,15]$.

Die Protonenmagnetresonanzspektroskopie ( $\left.{ }^{1} \mathrm{H}-\mathrm{MRS}\right)$ ermöglicht die nichtinvasive Quantifizierung von an den Stoffwechselprozessen beteiligten Metaboliten und Neurotransmittern und eröffnet dadurch eine weitere Dimension für die Erforschung zentraler Schmerzverarbeitung bzw. ihrer Dysfunktion bei der Entstehung chronischer Schmerzen. Besonders wichtig sind in diesem $\mathrm{Zu}$ sammenhang die erregend und hemmend wirkenden Neurotransmitter Glutamat und $\gamma$-Aminobuttersäure (GABA), die beide eine zentrale Rolle bei der Regulation neuronaler Aktivität spielen und deren Veränderungen als Ursache für die funktionellen Defizite während der Schmerzchronifizierung vermutet werden. Diesbezüglich konnten in früheren Studien erhöhte Glutamatkonzentrationen im anterioren cingulären Kortex (ACC), posterioren insulären Kortex und in der Amygdala sowie erniedrigte GABA-Konzentrationen im insulären Kortex von Schmerzpatienten nachgewiesen werden $[6,10]$.

Ziel dieser Studie war die spektroskopiebasierte Quantifizierung der Neurotransmitter bei Patienten mit unspezifischen chronischen Rücken- schmerzen und gesunden Kontrollen in zwei schmerzverarbeitenden Hirnregionen (ACC, Insula) und die Einbeziehung von klinischen und psychologischen Befunden in die Analyse.

\section{Hypothesen}

1. Im Vergleich zu gesunden Kontrollpersonen weisen Patienten erhöhte Glutamat- und verringerte GABAKonzentrationen auf.

2. Depressivität und Angst stehen im Zusammenhang mit veränderten Glutamat- und GABAKonzentrationen.

3. Klinische Merkmale (Schmerzintensität, Schmerzdauer, Chronifizierungsstadium) weisen einen Zusammenhang zu veränderten Glutamat- und GABAKonzentrationen auf.

\section{Material und Methoden}

\section{Stichprobe}

Im Rahmen einer Querschnittsuntersuchung wurden 19 Patienten mit chronischen unspezifischen Rückenschmerzen (CURS; 13 weiblich/6 männlich; Altersdurchschnitt: 55,3 Jahre) und 19 


\begin{tabular}{|c|c|c|}
\hline \multirow{2}{*}{$\begin{array}{l}\text { Schmerzintensität } \\
\text { (VAS) }\end{array}$} & $\mathrm{MW} \pm \mathrm{SD}$ & $6,3 \pm 1,3$ \\
\hline & Range & $4-9$ \\
\hline \multirow{2}{*}{$\begin{array}{l}\text { Schmerzdauer } \\
\text { (Jahre) }\end{array}$} & $\mathrm{M} \pm \mathrm{SD}$ & $13,3 \pm 12,1$ \\
\hline & Range & $1-41$ \\
\hline$\leq 5$ Jahre & $n(\%)$ & $8(42,1)$ \\
\hline$>5$ Jahre & $n(\%)$ & $10(52,6)$ \\
\hline Keine Angabe & $n(\%)$ & $1(5,3)$ \\
\hline \multicolumn{3}{|l|}{$\begin{array}{l}\text { Chronifizierungs- } \\
\text { stadium }\end{array}$} \\
\hline MPSS-Stadium I & $n(\%)$ & $2(10,5)$ \\
\hline MPSS-Stadium II & $n(\%)$ & $9(47,4)$ \\
\hline MPSS-Stadium III & $n(\%)$ & $8(42,1)$ \\
\hline \multicolumn{3}{|l|}{$\begin{array}{l}\text { Schmerz- } \\
\text { lokalisation }\end{array}$} \\
\hline Monolokulär & $n(\%)$ & $1(5,3)$ \\
\hline Bilokulär & $n(\%)$ & $10(52,6)$ \\
\hline Multilokulär & $n(\%)$ & $8(42,1)$ \\
\hline \multicolumn{3}{|l|}{$\begin{array}{l}\text { Medikamentenein- } \\
\text { nahme }\end{array}$} \\
\hline Nein & $n(\%)$ & $5(26,3)$ \\
\hline $\begin{array}{l}\text { Ja (Mehrfach- } \\
\text { nennung möglich) }\end{array}$ & $n(\%)$ & $14(73,7)$ \\
\hline Nicht bekannt & $n(\%)$ & $4(21,1)$ \\
\hline COX-Hemmer & $n(\%)$ & $6(31,6)$ \\
\hline Coxibe & $n(\%)$ & $3(15,8)$ \\
\hline Metamizol & $n(\%)$ & $5(26,3)$ \\
\hline Antikonvulsiva & $n(\%)$ & $7(36,8)$ \\
\hline \multicolumn{3}{|c|}{$\begin{array}{l}\text { COX Cyclooxygenase; MPSS Mainz Pain Staging } \\
\text { System; MW Mittelwert; SD Standardabweichung; } \\
\text { VAS visuelle Analogskala. }\end{array}$} \\
\hline
\end{tabular}

\begin{tabular}{|lll}
\hline \multicolumn{3}{|l}{ Tab. 2 Schmerzlokalisation } \\
\hline $\begin{array}{l}\text { Monolokulär } \\
\text { (unterer Rücken) }\end{array}$ & $n(\%)$ & $1(5,3)$ \\
\hline $\begin{array}{l}\text { Bilokulär } \\
\text { (Zweifachnennungen) }\end{array}$ & $n(\%)$ & $\begin{array}{l}10 \\
(52,6)\end{array}$ \\
\hline Nacken, Schultern & $n(\%)$ & $4(40,0)$ \\
\hline Oberer Rücken & $n(\%)$ & $2(20,0)$ \\
\hline Unterer Rücken & $n(\%)$ & $\begin{array}{l}10 \\
(100,0)\end{array}$ \\
\hline $\begin{array}{l}\text { Gesäß (Ausstrahlung in } \\
\text { untere Extremitäten) }\end{array}$ & $n(\%)$ & $4(40,0)$ \\
\hline $\begin{array}{l}\text { Multilokulär (Mehrfach- } \\
\text { nennungen möglich) }\end{array}$ & $n(\%)$ & $8(42,1)$ \\
\hline Nacken, Schultern & $n(\%)$ & $7(77,8)$ \\
\hline Oberer Rücken & $n(\%)$ & $6(66,7)$ \\
\hline $\begin{array}{l}\text { Unterer Rücken } \\
\text { Gesäß (Ausstrahlung in }\end{array}$ & $n(\%)$ & $\begin{array}{l}9(77,8) \\
(100,0)\end{array}$ \\
\hline \begin{tabular}{l} 
untere Extremitäten) \\
\hline
\end{tabular} & &
\end{tabular}

\section{Der Schmerz}

nach Alter und Geschlecht parallelisierte, gesunde Kontrollpersonen [,healthy control" (HC); 13 weiblich/6 männlich; Altersdurchschnitt: 53,8 Jahre] untersucht. Weitere demografische Befunde der Schmerzpatienten sind - Tab. $1 \mathrm{zu}$ entnehmen.

Das Einschlusskriterium war Rückenschmerz ohne spezifische Genese (z. B. Bandscheibenvorfälle, Osteoporose, Frakturen und Operationen im Rückenbereich) seit mehr als 3 Monaten mit einer durchschnittlichen Intensität $>4$ auf der visuellen Analogskala (VAS). Ausgeschlossen wurden Patienten mit Nikotinkonsum vor der Untersuchung, mit schwerwiegenden psychiatrischen oder neurodegenerativen Erkrankungen, mit regelmäßiger Psychopharmako- oder Opiattherapie sowie mit allgemeinen MRT-Kontraindikationen.

\section{Magnetresonanzspektroskopie}

Alle Messungen wurden an einem klinischen 3-T-Ganzkörper-MR-Scanner durchgeführt (TIM Trio, Siemens Healthcare, Erlangen). Das Untersuchungsprotokoll (etwa $40 \mathrm{~min}$ ) beinhaltete die Akquisition von räumlich hochaufgelösten anatomischen MR-Bilddaten des Gehirns sowie von jeweils zwei Protonenspektren im ACC und in der Insula (- Abb. 1c). Das erste Spektrum wurde mit einer konventionellen ${ }^{1} \mathrm{H}$-MRSpoint-resolved-spectroscopy(PRESS)Sequenz akquiriert [Echozeit (TE)/ Repetitionszeit (TR) $=30 / 1800 \mathrm{~ms}$; - Abb. 1a]. Aus den Flächen unter den in - Abb. 1a beschrifteten Peaks wurden in diesem Spektrum die wichtigsten Hirnmetaboliten N-Acetyl-Aspartat (NAA; Neuronendichtemarker), myo-Inositol (mI; Gliazellendichtemarker), Gesamtkreatin (tCr; Energiestoffwechselmarker) und Gesamtcholin (tCho; Marker für den Membranstoffwechsel) sowie das Summensignal des erregend wirkenden Neurotransmitters Glutamat und des glutamatergen Zwischenprodukts Glutamin (Glx) quantifiziert.

Für die Akquisition des zweiten Spektrums wurde eine spezielle Spectralediting-1 ${ }^{1} \mathrm{H}$-MRS-MEGA-PRESS-Sequenz verwendetet (MEGA-PRESS; TE/ $\mathrm{TR}=68 / 2000 \mathrm{~ms} ; \bullet$ Abb. 1b), die es ermög- licht, den üblicherweise durch andere Metabolitensignale überlagerten Peak des hemmend wirkenden Neurotransmitters GABA zu quantifizieren [16]. Anschließend wurden die Intensitäten von Glx und GABA mit der tCrIntensität normiert ( $\mathrm{Glx} / \mathrm{tCr}$ - und GABA/tCr-Verhältnisse), die bei ${ }^{1} \mathrm{H}$ MRS-Messungen in vivo im Gehirn als endogene Referenz verwendet wird. Des Weiteren wurde der häufig als Marker für das Gleichgewicht zwischen Erregung und Hemmung herangezogene Quotient aus Glx und GABA (Glx/GABA) aus den Spektren bestimmt [11].

\section{Messung soziodemografischer und klinischer Parameter}

Zur Erfassung psychischer Störungen nach dem Diagnostic and Statistical Manual of Mental Disorders (DSM-IV) wurde mit allen teilnehmenden Probanden ein strukturiertes klinisches Interview (SKID I und II) durchgeführt [23]. Als Grundlage für die Erhebung der soziodemografischen (Alter, Geschlecht, Bildungsstand) und klinischen Parameter (Schmerzintensität, Schmerzdauer, funktionelle Beeinträchtigung, Chronifizierungsstadium) diente der Deutsche Schmerzfragebogen [18]. Das Chronifizierungsstadium wurde mithilfe des Mainz Pain Staging System (MPSS; [12]) ermittelt. Zuletzt wurden Angst und Depression mit der deutschen Version der Hospital Anxiety and Depression Scale (HADS-D und HADS-A; [4]) erfasst.

\section{Statistische Auswertung}

Alle statistischen Analysen erfolgten mit SPSS 21.0. In Anlehnung an die erste Hypothese unserer Studie wurden alle erhobenen klinischen Parameter sowie die gemessenen Glx/tCr-, und GABA/ tCr-Quotienten zwischen den Patienten und gesunden Kontrollen verglichen. Diese Quotienten wurden zunächst hinsichtlich ihrer Verteilungseigenschaften untersucht (Kolmogorow-SmirnovTest) und auf Unterschiede zwischen den parallelisierten Gruppen mittels t-Test für gepaarte Stichproben (Signifikanzniveau von $p \leq 0,05)$ getestet und nach Bonferroni korrigiert. Effektgrößen für 
Schmerz DOI 10.1007/s00482-015-0082-5

(๑) Deutsche Schmerzgesellschaft e.V. Published by Springer-Verlag Berlin Heidelberg - all rights reserved 2015

L. Janetzki · A. Gussew · R. Malessa · U. Habenicht · J.R. Reichenbach · B. Strauß · C. Borys

Hirnmetabolische Veränderungen bei chronischem Rückenschmerz. Studie unter Berücksichtigung von klinischen und psychischen Parametern

Zusammenfassung

Hintergrund. Die Chronifizierung von Schmerzen und die damit verbundenen psychologischen Beeinträchtigungen stehen im Zusammenhang mit Änderungen des Neurotransmitterstoffwechsels in schmerzverarbeitenden Hirnregionen, z. B. dem anterioren cingulären Kortex (ACC) und insulären Kortex. In dieser Studie wurde die Magnetresonanzspektroskopie ('H-MRS) genutzt, um Neurotransmitter im Gehirn in vivo zu quantifizieren und ein schmerzbegleitendes Ungleichgewicht zwischen erregend (glutamatergen) und hemmend wirkenden (GABAergen) Neurotransmittern bei Patienten mit chronischen unspezifischen Rückenschmerzen nachzuweisen sowie den Zusammenhang zu psychologischen und klinischen Befunden zu untersuchen.

\begin{abstract}
Material und Methoden. Bei 19 Patienten mit chronischen unspezifischen Schmerzen (>3 Monate) im Rückenbereich sowie bei 19 nach Alter und Geschlecht parallelisierten gesunden Kontrollpersonen wurden einerseits soziodemografische, psychologische und Schmerzmerkmale mittels Fragebogen erfasst, andererseits wurden Glutamat und GABA sowie Glutamat/GABA-Verhältnisse im ACC und im insulären Kortex mittels ${ }^{1} \mathrm{H}$-MRS quantifiziert.

Ergebnisse. Glutamat/GABA-Verhältnisse von Patienten und Kontrollpersonen zeigten eine hohe Varianz. Ein signifikanter Unterschied konnte nicht nachgewiesen werden. Signifikante Einflussvariablen im Regressionsmodell waren Ängstlichkeit als Prädiktor für eine Glutamatverringerung sowie Depressivi-
\end{abstract}

tät und Alter als Prädiktoren für reduziertes GABA im ACC. In der Patientengruppe zeigte sich ein signifikanter Einfluss der Schmerzstärke auf GABA und Glutamat in der Insula. Schlussfolgerungen. Trotz der einheitlichen Diagnose „chronischer unspezifischer Rückenschmerz" zeigte sich eine große Varianz der Neurotransmitter in den schmerzverarbeitenden Hirnregionen, die nur unter Einbeziehung klinischer und psychologischer Parameter wie Schmerzintensität und Depressivität erklärt werden kann.

\section{Schlüsselwörter}

Chronischer Schmerz · $\gamma$-Aminobuttersäure . Glutamat Psychologische Faktoren .

Protonenmagnetresonanzspektroskopie

\section{Cerebral metabolic changes and chronic back pain. Study taking into consideration clinical and psychological parameters}

\section{Abstract}

Background. The manifestation of chronic pain and psychological impairments are related to alterations of neurotransmitter metabolism in cerebral pain processing regions, e.g., anterior cingular cortex (ACC), insula. Magnetic resonance spectroscopy ( $\left.{ }^{1} \mathrm{H}-\mathrm{MRS}\right)$ enables in vivo quantification of neurotransmitters in the brain and was applied in this study to examine the hypothesized chronic pain-related imbalance between excitatory (glutamatergic) and inhibitory (GABA-ergic) neurotransmitter turnovers in the brain of patients with nonspecific chronic pain.

Materials and methods. A total of $19 \mathrm{pa}-$ tients with nonspecific chronic ( $>3$ months) back pain and 19 age- and gender-matched healthy subjects participated in this study. Glutamate and GABA as well as glutamate/ GABA ratios were determined in the $A C C$ and insula using ${ }^{1} \mathrm{H}-\mathrm{MRS}$. Sociodemographic, psychological, and pain-related features were measured with standardized questionnaires. Results. There was a strong variance of glutamate/GABA ratios for both patients and healthy subjects with no significant difference between the two groups. Regression analysis revealed certain significant predictors, such as anxiety as causal variable for reduced glutamate and depression and age as predictors for reduced GABA in ACC. In the patient group, intensity of pain was a signif- icant predictor for glutamate and GABA levels in the insula.

Conclusions. Despite the uniform diagnosis of nonspecific chronic back pain, we observed a strong variance of neurotransmitters in cerebral pain processing regions. It is necessary to include psychological as well as clinical parameters (e.g., intensity of pain or depression) for a proper interpretation of neurotransmitter turnovers.

\section{Keywords}

Chronic pain $\cdot \gamma$-aminobutyric acid .

Glutamate - Psychological factors .

Proton magnetic resonance spectroscopy
Mittelwertunterschiede wurden mittels Cohens d bestimmt [5].

Im zweiten Schritt wurden Abhängigkeiten zwischen Glx/tCr- und GABA/ tCr-Quotienten (abhängige Variable) und klinischen Parametern mittels multipler Regressionsanalysen (schrittweise rückwärts) untersucht. Im ersten Regressionsmodell wurden Glx/tCr und GABA/ tCr aus der gesamten Stichprobe (HC und CURS) unter Einbeziehung der erklärenden Variablen Alter, Geschlecht, Angst und Depression analysiert. Im zweiten Regressionsmodell wurden die Abhängigkeiten zwischen Neurotransmitterquotienten und Schmerzdauer, durchschnittlicher Schmerzstärke und Chronifizierungsstadium ausschließlich für die Patientengruppe untersucht. Für die durchschnittliche Schmerzdauer wurde zur Wichtung von Ausreißern eine Dummy-Variable mit den Ausprägungen bis einschließlich 5 Jahre und länger als 5 Jahre eingeführt. Das Signifikanzniveau wurde auf 0,05 festgelegt. Zusätzlich wurde auf Multikollinearität (Toleranzwert $>0,25$, Varianzinflationsfaktor <5; [21]) geprüft.

\section{Ergebnisse}

Die mittlere Schmerzintensität lag bei 6,3 $\pm 1,3$ (Maximum: 9), die durchschnittliche Schmerzdauer betrug 13,3 $\pm 12,1$ Jahre. Die meisten Patienten waren im Chronifizierungsstadium II; 18 von 19 Patienten wiesen $\geq 2$ Schmerzlokalisationen im Rückenbereich auf (• Tab. 2). 


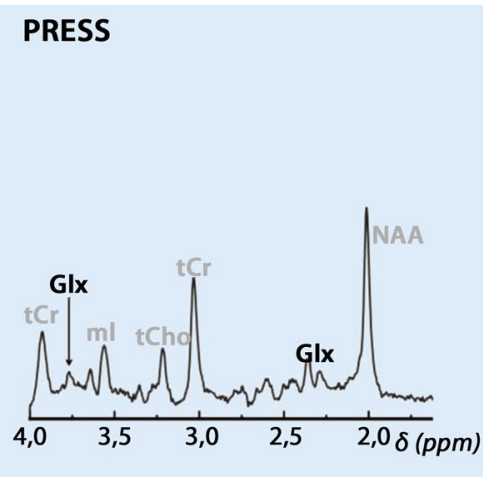

a

\section{MEGA-PRESS}

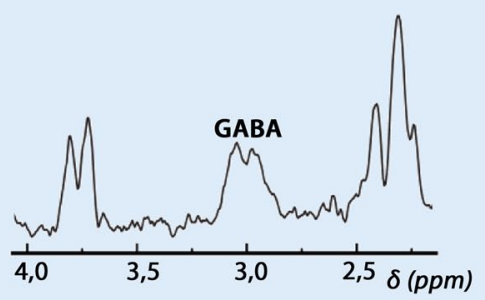

b

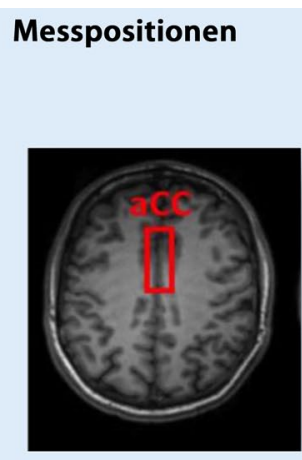

d
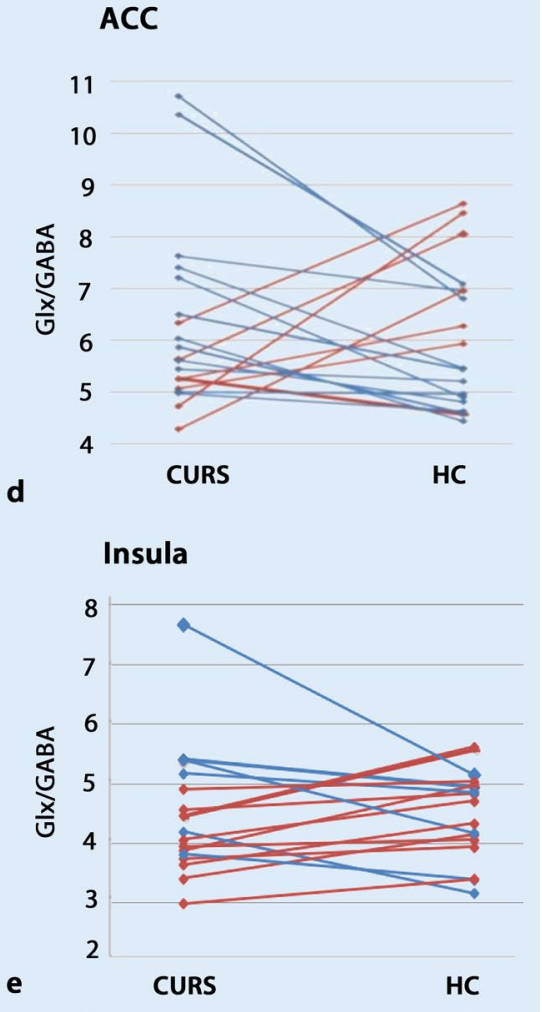

Abb. $1 \Delta$ Repräsentative PRESS- (a) und MEGA-PRESS- ${ }^{1} \mathrm{H}-$ Magnetresonanzspektren (b) aus dem ACC einer Patientin (a und b) sowie Darstellung der Magnetresonanzspektroskopie(MRS)-Voxelpositionen im ACC und in der linken Insula (c). Ergebnisse: Glx/GABA-Quotienten von korrespondierenden Schmerzpatienten (CURS) und Kontrollpersonen (HC) im ACC (d) und in der Insula (e). Verbindungslinien kennzeichnen korrespondierende CURS-HC-Paare (GIx/GABACURS $>$ Glx/GABA $A_{H C}$ und GIx/GABA $\left.A_{C U R S}<\mathrm{Glx} / G A B A_{H C}\right)$. ACC Anteriorer cingulärer Kortex; CURS Patienten mit chronischen unspezifischen Rückenschmerzen; GABA y-Aminobuttersäure; Glx Glutamat und Glutamin; $H C_{\text {"I }}$ healthy controls" (gesunde Kontrollpersonen); PRESS "point resolved spectroscopy"; tCho Gesamtcholin; tCr Gesamtkreatin; NAA N-Acetyl-Aspartat; mI myo-Inositol

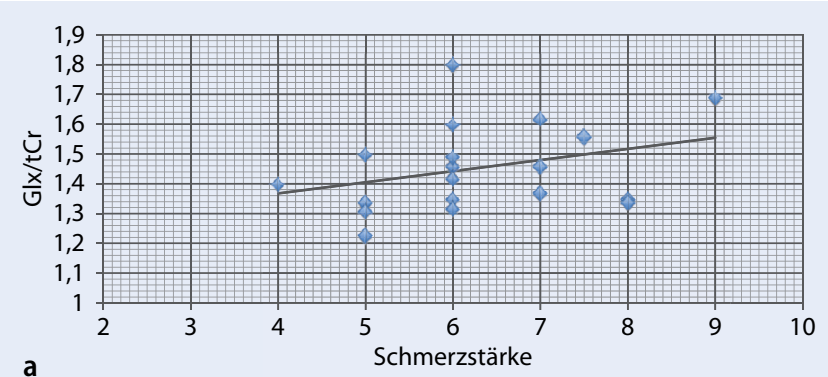

a

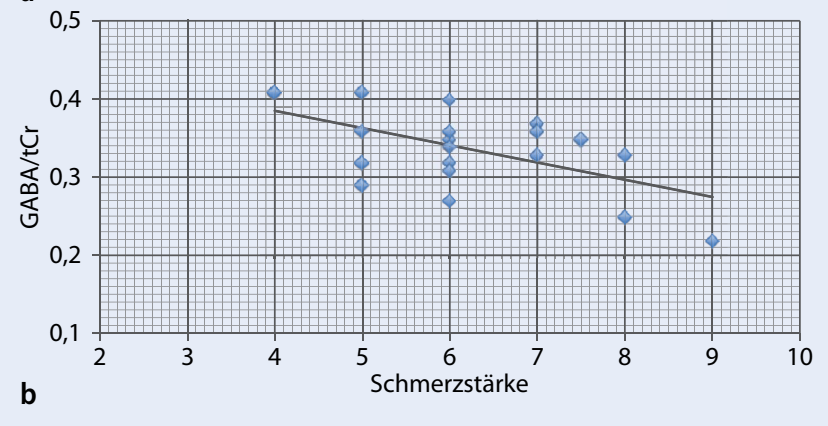

Abb. $2<$ Verteilung von a $\mathrm{Glx}(\mathrm{Glx} / \mathrm{tCr})$ und $\mathbf{b}$ GABA (GABA) $\mathrm{tCr}$ ) in der Insula in Abhängigkeit von der Schmerzstärke (visuelle Analogskala) mit korrespondierenden linearen Regressionsgeraden. GABA YAminobuttersäure; Glx Glutamat und Glutamin; $t C r$ Gesamtkreatin

Auf der HADS gaben Schmerzpatienten im Vergleich zu Kontroll- personen signifikant erhöhte Werte für Depressivität und Angst an (• Tab. 3).
Vergleich von Neurotransmittern der Patienten- und Kontrollgruppe (Hypothese 1)

Während - Abb. 1d und e die Verteilungen der Glx/GABA-Quotienten für die gepaarten Untersuchungsgruppen in den beiden untersuchten Hirnarealen darstellen, enthält $\bullet$ Tab. 3 die mittleren Glx/tCr- und GABA/tCr- sowie Glx/ GABA-Quotienten bei Patienten mit CURS und gesunden Kontrollen (HC) zusammen mit Ergebnissen der Mittelwertvergleiche.

Insgesamt weisen die Werte für Glx/ GABA in beiden Gruppen eine starke Varianz auf. Im Unterschied zur Insula zeigte sich im ACC ein Trend von erhöhten Glx/GABA-Quotienten bei CURS (• Abb. 1d, e), der sich v. a. auf tendenziell erhöhte Glx/tCr-Quotienten zurückführen lässt (vgl. • Tab. 3). Beim Mittelwertvergleich zeigten sich jedoch weder 
Tab. 3 Stichprobenbeschreibung

\begin{tabular}{|c|c|c|c|c|c|c|}
\hline & & CURS $(n=19)$ & $\mathrm{HC}(n=19)$ & $\begin{array}{l}\text { Effekt- } \\
\text { stärke }\end{array}$ & $p$-Werte & $95 \%-\mathrm{KI}$ \\
\hline $\begin{array}{l}\text { Depressivität } \\
\text { (HADS-D) }\end{array}$ & $\begin{array}{l}\mathrm{MW} \pm \mathrm{SD} \\
\text { (Range) }\end{array}$ & $5,9 \pm 4,4(1-18)$ & $1,5 \pm 1,8(0-6)$ & 1,3 & 0,001 & $(-6,0,-1,8)$ \\
\hline $\begin{array}{l}\text { Ängstlichkeit } \\
\text { (HADS-A) }\end{array}$ & $\begin{array}{l}\mathrm{MW} \pm \mathrm{SD} \\
\text { (Range) }\end{array}$ & $6,7 \pm 3,2(1-14)$ & $2,8 \pm 2,2(0-14)$ & 1,5 & 0,001 & $(-6,8,-2,2)$ \\
\hline
\end{tabular}

Tab. 4 Übersicht der mittleren Neurotransmitterkonzentrationen nach Normierung mit

Kreatin als $\mathrm{Glx} / \mathrm{tCr}-$, GABA/tCr- und GIx/GABA-Quotienten im ACC und in der Insula von CURS-

Patienten und $\mathrm{HC}$ zusammen mit den mittels t-Tests für abhängige Stichproben berechneten

p-Werten und Effektstärken

\begin{tabular}{|llllll}
\hline Hirnregion & $\begin{array}{l}\text { Neurotrans- } \\
\text { mitter }\end{array}$ & $\begin{array}{l}\text { CURS } \\
\text { (MW } \pm \text { SD) }\end{array}$ & HC (MW \pm SD) & $p$-Wert & Effektstärke \\
\hline $\begin{array}{l}\text { Anteriorer } \\
\text { cingulärer Kortex }\end{array}$ & $\mathrm{Glx} / \mathrm{tCr}$ & $1,5 \pm 0,1$ & $1,5 \pm 0,1$ & 0,8 & 0,1 \\
\cline { 2 - 6 } & $\mathrm{GABA} / \mathrm{tCr}$ & $0,2 \pm 0,01$ & $0,2 \pm 0,0$ & 0,9 & 0,0 \\
\cline { 2 - 6 } & $\mathrm{Glx} / \mathrm{GABA}$ & $6,2 \pm 1,5$ & $6,0 \pm 1,5$ & 0,6 & 0,2 \\
\hline \multirow{3}{*}{ Insula } & $\mathrm{Glx} / \mathrm{tCr}$ & $1,5 \pm 0,1$ & $1,4 \pm 0,2$ & 0,5 & 0,1 \\
\cline { 2 - 6 } & $\mathrm{GABA} / \mathrm{tCr}$ & $0,3 \pm 0,1$ & $0,3 \pm 0,0$ & 0,4 & 0,2 \\
\cline { 2 - 6 } & $\mathrm{Glx} / \mathrm{GABA}$ & $4,5 \pm 1,1$ & $4,5 \pm 1,4$ & 0,9 & 0,0 \\
\hline
\end{tabular}

$\mathrm{Gl} / \mathrm{t} \mathrm{Cr}$ Erregend wirkende Neurotransmitter als Summe von Glutamat und Glutamin/Kreatin; $G A B A / t C r$ hemmend wirkende Neurotransmitter als GABA/Kreatin.

ACC Anteriorer cingulärer Kortex; CURS Patienten mit chronischen unspezifischen Rückenschmerzen; GABA YAminobuttersäure; GIx Glutamat und Glutamin; $H C_{\text {"I }}$ healthy controls" (gesunde Kontrollpersonen); $M W$ Mittelwert; SD Standardabweichung; $t C r$ Gesamtkreatiny.Y

Tab. 5 Multiple Regression: Neurotransmitter und psychische Faktoren

\begin{tabular}{|c|c|c|c|c|c|c|c|c|}
\hline & & Variablen & b & $\beta$ & $\mathbf{t}$ & $\begin{array}{l}\text { Signi- } \\
\text { fikanz }\end{array}$ & Toleranz & $\begin{array}{l}\text { Varianz- } \\
\text { inflations- } \\
\text { faktor }\end{array}$ \\
\hline \multirow[t]{4}{*}{$\mathrm{Glx} / \mathrm{tCr}$} & \multirow[t]{2}{*}{ ACC } & $\begin{array}{l}\text { Ängstlichkeit } \\
\text { (HADS-A) }\end{array}$ & $-0,01$ & $-0,30$ & $-1,86$ & 0,07 & 1,00 & 1,00 \\
\hline & & \multicolumn{7}{|c|}{$R=0,30 ; R^{2}=0,09 ; F=3,46$} \\
\hline & \multirow[t]{2}{*}{ Insula ${ }^{a}$} & Alter (Jahre) & 0,01 & 0,34 & 2,17 & 0,04 & 1,00 & 1,00 \\
\hline & & \multicolumn{7}{|c|}{$\mathrm{R}=0,34 ; \mathrm{R}^{2}=0,12 ; \mathrm{F}=4,72$} \\
\hline \multirow[t]{5}{*}{$\mathrm{GABA} / \mathrm{tCr}$} & \multirow[t]{3}{*}{$\mathrm{ACC}^{\mathrm{a}}$} & $\begin{array}{l}\text { Depressivität } \\
\text { (HADS-D) }\end{array}$ & $-0,02$ & $-0,26$ & $-1,71$ & 0,05 & 0,98 & 1,02 \\
\hline & & Alter (Jahre) & $-0,01$ & $-0,45$ & $-2,99$ & 0,01 & 0,98 & 1,02 \\
\hline & & $R=0,48 ; R^{2}=0$, & $F=5,34$ & & & & & \\
\hline & \multirow[t]{2}{*}{ Insula ${ }^{a}$} & Alter (Jahre) & $-0,01$ & $-0,33$ & $-2,10$ & 0,04 & 1,00 & 1,00 \\
\hline & & \multicolumn{7}{|c|}{$R=0,33 ; R^{2}=0,11 ; F=4,42$} \\
\hline \multicolumn{9}{|c|}{$\begin{array}{l}\text { aModell ist signifikant bei } p<0,05 \text {. } \\
\text { GIx/tCr Erregend wirkende Neurotransmitter als Summe von Glutamat und Glutamin/Kreatin; GABA/tCr } \\
\text { hemmend wirkende Neurotransmitter als GABA/Kreatin. } \\
\text { ACC Anteriorer cingulärer Kortex; GABA } y \text {-Aminobuttersäure; Glx Glutamat und Glutamin; HADS Hospital } \\
\text { Anxiety and Depression Scaley; } \gamma t C r \text { Gesamtkreatinץ.Y }\end{array}$} \\
\hline
\end{tabular}

im ACC noch in der Insula signifikante Glx/tCr-, GABA/tCr- oder Glx/GABAUnterschiede zwischen CURS-Patienten und HC (- Tab. 4).

\section{Psychologische Faktoren und} Neurotransmitter (Hypothese 2)

In - Tab. 5 sind die Ergebnisse der linearen Regressionsanalyse zur Varianzaufklärung für $\mathrm{Glx} / \mathrm{tCr}$ und $\mathrm{GABA} /$ $\mathrm{tCr}$ im ACC und in der Insula von
Patienten und Kontrollen zusammengefasst. Prädiktoren für $\mathrm{GABA} / \mathrm{tCr}$ im ACC waren Depressivität $(\beta=-0,26)$ und Alter $(\beta=-0,45)$ mit einer Varianzaufklärung von $23 \%\left(R^{2}=0,23\right)$. Dagegen wurde Angst als marginal signifikanter Prädiktor für Glx/tCr im ACC $(\beta=-0,30)$ bei einer Varianzaufklärung von lediglich $9 \%\left(\mathrm{R}^{2}=0,09\right)$ bestimmt. Das Alter erwies sich außerdem als Einflussfaktor auf Glx/ $\operatorname{tCr}\left(\beta=0,34 ; \mathrm{R}^{2}=0,12\right)$ und $\mathrm{GABA} / \mathrm{tCr}$ in der Insula $\left(\beta=-0,33 ; R^{2}=0,11\right)$.

\section{Klinische Parameter und Neurotransmitter (Hypothese 3)}

In - Abb. 2a sind für die Schmerzpatienten Glx/tCr und in - Abb. 2b GABA/tCr in der Insula in Abhängigkeit von der Schmerzstärke aufgetragen. Während Glx/tCr mit steigender Schmerzintensität zunimmt, zeigt sich eine Abnahme von GABA/tCr bei steigender Schmerzstärke (• Abb. 2).

In - Tab. 6 sind die Ergebnisse der Regressionsanalyse zur Varianzaufklärung der Neurotransmitterparameter bei CURS-Patienten in Abhängigkeit von den Prädiktoren Chronifizierungsstadium, Schmerzdauer und -intensität sowie Alter und Geschlecht dargestellt.

Das Chronifizierungsstadium $(\beta=-0,49)$ klärt insgesamt $19 \%\left(\mathrm{R}^{2}=0,19\right)$ der Varianz von $\mathrm{Glx} / \mathrm{tCr}$ im ACC auf. In der Insula trägt die Schmerzstärke $(\beta=0,41)$ neben dem Geschlecht $(\beta=-0,41)$ marginal signifikant $(p=0,09)$ zu einer Varianzaufklärung von $\mathrm{Glx} / \mathrm{tCr}$ von $31 \%\left(R^{2}=0,27\right)$ bei, Prädiktoren für $\mathrm{GABA} / \mathrm{tCr}$ in der Insula waren die Schmerzstärke $(\beta=-0,65)$ und das Alter $(\beta=-0,49)$. Die Varianzaufklärung des Modells lag bei $54 \%\left(\mathrm{R}^{2}=0,54\right)$.

\section{Diskussion}

Ziel unserer Studie war es, Veränderungen der zentralen Botenstoffe GABA und Glutamat im Gehirn von chronischen Rückenschmerzpatienten unter Berücksichtigung klinischer und psychologischer Parameter zu untersuchen. 
Tab. 6 Zusammenhang zwischen soziodemografischen Merkmalen, schmerzbezogenen Faktoren und Hirnregionen

\begin{tabular}{|c|c|c|c|c|c|c|c|c|}
\hline & & Variablen & b & $\beta$ & $t$ & Signifikanz & Toleranz & Varianzinflationsfaktor \\
\hline \multirow[t]{5}{*}{$\mathrm{Glx} / \mathrm{tCr}$} & \multirow[t]{2}{*}{ ACC } & $\begin{array}{l}\text { Chronifizierungsstadium } \\
\text { (MPSS) }\end{array}$ & $-0,94$ & $-0,49$ & $-2,25$ & 0,04 & 1,00 & 1,00 \\
\hline & & $R=0,49 ; R^{2}=0,19 ; F=5,07$ & & & & & & \\
\hline & \multirow[t]{3}{*}{ Insula } & Geschlecht & $-0,13$ & $-0,41$ & $-1,83$ & 0,09 & 0,95 & 1,05 \\
\hline & & Schmerzstärke (VAS) & 0,05 & 0,41 & 1,82 & 0,09 & 0,95 & 1,05 \\
\hline & & $R=0,51 ; R^{2}=0,27 ; F=42,73$ & & & & & & \\
\hline \multirow[t]{3}{*}{$\mathrm{GABA} / \mathrm{tCr}$} & \multirow[t]{3}{*}{ Insula } & Alter (Jahre) & $-0,01$ & $-0,49$ & $-2,96$ & 0,01 & 0,99 & 1,01 \\
\hline & & Schmerzstärke (VAS) & $-0,03$ & $-0,65$ & $-3,89$ & 0,00 & 0,99 & 1,01 \\
\hline & & $R=0,77 ; R^{2}=0,54 ; F=10,85$ & & & & & & \\
\hline
\end{tabular}

Glx/tCr Erregend wirkende Neurotransmitter als Summe von Glutamat und Glutamin/Kreatin; GABA/tCr hemmend wirkende Neurotransmitter als GABA/Kreatin. ACC Anteriorer cingulärer Kortex; GABA y-Aminobuttersäure; GIx Glutamat und Glutamin; MPSS Mainz Pain Staging Systemץ; $\gamma t C r$ Gesamtkreatiny.Y

\section{Vergleich von Neurotransmittern der Patienten- und Kontrollgruppe}

Es konnte kein signifikanter Unterschied der Neurotransmitterquotienten zwischen Patienten und Kontrollpersonen nachgewiesen werden. Im ACC wurden bei CURS im Vergleich zu HC tendenziell erhöhte Glx/GABAQuotienten gemessen. Besonders in der Patientengruppe (• Tab. 5) zeigten die Neurotransmitter in beiden Hirnregionen eine starke Varianz, z. B. Glx/GABA im ACC: $S D=1,55$; Range $=4,28-10,71$.

Hierbei ist zu berücksichtigen, dass die Patienten v. a. bezüglich der Schmerzdauer (Range =1-41; SD =12,1; • Tab. 5) und durchschnittlichen Schmerzstärke (Range $=4-9 ; \mathrm{SD}=1,3$; • Tab. 5) ein breites Spektrum aufwiesen. Unter dem Begriff „chronischer unspezifischer Rückenschmerz" wird eine sehr komplexe, heterogene Patientengruppe zusammengefasst [24]. Die Diagnose ist allein stehend nicht geeignet, um neurochemische Befunde zu erklären und eine Aussage über eine potenzielle Entgleisung des Glx/GABA-Verhältnisses zu treffen. Grund hierfür ist u. a. die spezifische Wirkung des Neurotransmitters Glutamat. Bisher wurden in der Mehrheit der Studien bei Schmerzpatienten ein Anstieg von Glutamat und eine Abnahme von GABA beobachtet $[6,11]$. Glutamat hat in hohen Konzentrationen einen exzitotoxischen Effekt: Ein massiver Kalziumeinstrom führt zum Zelltod glutamaterger Neurone $[4,9]$. In Abhängigkeit von der Dauer, der Intensität der Schmerzen oder dem Chronifizierungsstadium könnte es somit zunächst zu einer überschießenden
Exzitation und im Verlauf zum Verlust glutamaterger Neurone kommen.

Des Weiteren liegt eine Reihe psychischer Faktoren vor (Angst, Depressivität), deren Einfluss auf Glutamat und GABA berücksichtigt werden muss $[8,20]$. Jeder Patient bringt hier eine Reihe individueller Befunde mit. Die Heterogenität der Patientengruppe bezüglich aller o. g. Parameter ist ebenso charakterisierend für das Krankheitsbild wie auch herausfordernd für Studien in diesem Bereich.

\section{Psychologische Faktoren und Neurotransmitter}

Diese individuellen Befunde wurden in den nächsten beiden Schritten berücksichtigt. Psychische Faktoren wie Depressivität, schmerzbezogene Kognitionen (Angst, Katastrophisieren, Hilf- und Hoffnungslosigkeit) und passives Schmerzverhalten gelten laut Leitlinie als Risikofaktoren („yellow flags") für die Chronifizierung von Schmerzen [2]. Die untersuchte Patientengruppe zeigte im Vergleich zu gesunden Kontrollpersonen signifikant erhöhte Werte für Depressivität und Ängstlichkeit, wobei die Werte durchschnittlich unter dem klinischen „cut-off“ $(\geq 12)$ lagen. Es konnte gezeigt werden, dass mittels psychologischer Tests erfasste Komorbiditäten neurochemische Korrelate aufweisen. In Übereinstimmung mit früheren Studien konnten wir reduzierte GABA/tCr-Quotienten im ACC im Zusammenhang mit Depressivität $(\beta=-0,26)$ messen $[1,7]$. Erhöhte Ängstlichkeit ging mit kleineren Glx/tCr-Quotienten einher (• Tab. 3). Diese Ergebnisse decken sich mit Er- kenntnissen aus früheren Positronenemissionstomographie- und fMRTStudien, die nachwiesen, dass die Aktivität im ACC bei Schmerzpatienten wesentlich von affektiven Begleitzuständen der Schmerzen abhängig ist [17].

\section{Klinische Parameter und Neurotransmitter}

Bei der dritten Hypothese wurde vorausgesetzt, dass klinische Merkmale einen Zusammenhang zu den Ausprägungen der erfassten neurometabolischen Parameter aufweisen. In unserer Patientengruppe zeigte sich mit zunehmender Chronifizierung eine Abnahme des Neurotransmitters Glx/tCr im ACC ( $\beta=-0,49$; - Tab. 6). In Anbetracht des oben beschriebenen Mechanismus der Exzitotoxizität ist bei fortschreitender Chronifizierung mit einem zunehmenden Verlust erregender Neurone im ACC zu rechnen. Dieser Zusammenhang wird in unserer Studie erstmals beschrieben. Eine Verifizierung sollte in Folgestudien an größeren, über die Chronifizierungsstadien 1-3 normalverteilten Stichproben erfolgen.

In der Insula zeigte sich eine Abhängigkeit von Glx/tCr und GABA/tCr und der Schmerzstärke (• Abb. 2). Dieser Befund deckt sich mit Ergebnissen früherer fMRT-Studien, die eine erhöhte Aktivität im insulären Kortex in Abhängigkeit von sensorisch-diskriminativen Aspekten der Schmerzverarbeitung nachwiesen [17]. Aus den Ergebnissen lässt sich die Hypothese ableiten, dass den Hirnregionen ACC und Insula im Prozess der Schmerzchronifizierung unterschiedliche Rollen zukommen: Im ACC nahmen 
psychische Parameter (Ängstlichkeit, Depressivität) einen signifikanten Einfluss auf Hirnparameter, in der Insula die Schmerzintensität. Eine Überprüfung dieses Zusammenhangs soll in Folgestudien mit einer Kontrollgruppe depressiver Patienten im Vergleich zu chronischen Schmerzpatienten erfolgen.

\section{Limitationen und Ausblick}

Folgende Limitationen müssen bei dem vorliegenden Studiendesign berücksichtigt werden: Die in den ${ }^{1} \mathrm{H}-\mathrm{MR}$ Spektren quantifizierten Intensitäten repräsentieren Summenwerte der Metaboliten im Messvolumen. Glutamat ist jedoch nicht nur als Neurotransmitter aktiv, sondern auch am Energiestoffwechsel von Zellen als Metabolit des Citratzyklus beteiligt. Ein hohes Glutamatniveau kann deswegen sowohl für einen hohen Energieverbrauch als auch für ein hohes Erregungspotenzial der Zelle sprechen. Dies könnte eine Erklärung für den in dieser Studie ungeklärten Anteil der Varianz sein. Des Weiteren handelt es sich um eine kleine Stichprobe, sodass bezüglich der Parameter Schmerzdauer und Chronifizierungsstadium keine Normalverteilung erreicht werden konnte.

Die Vorgänge der zentralnervösen Schmerzverarbeitung und Chronifizierung zu verstehen, bleibt eine der größten Herausforderungen der aktuellen Schmerzforschung. Diese Studie soll in erster Linie der Hypothesengenerierung dienen und die Bedeutung einer ausführlichen, individuellen Befunderhebung bei Schmerzpatienten belegen.

Eine Reihe von Einflussfaktoren wurde im Zusammenhang mit schmerzbedingten hirnmetabolischen Veränderungen untersucht. Es bleibt jedoch offen, inwieweit die neurochemischen Veränderungen Folge oder Ursache chronischer Schmerzsyndrome sind. Prospektiv angelegte Untersuchungen mit umfangreicheren Stichproben und kontrollierten Untersuchungsbedingungen sind daher notwendig, um Kausalzusammenhänge und interagierende Einflussfaktoren ermitteln und zum Verständnis der zentralnervösen Schmerzverarbeitung beitragen zu können.

\section{Fazit für die Praxis}

- Die Heterogenität chronischer Schmerzpatienten bezüglich klinischer und psychologischer Befunde spiegelt sich in der Streuung der Neurotransmitter Glutamat und GABA wider.

- Psychische Komorbiditäten von Schmerzpatienten zeigen zentralnervöse Korrelate im ACC.

- Stoffwechselveränderungen in der Insula stehen im Zusammenhang mit klinischen Parametern.

- Prospektiv angelegte Studien sollten in Zukunft Kausalzusammenhänge zwischen Schmerzen und Glutamat sowie GABA untersuchen.

\section{Korrespondenzadresse}

\section{Janetzki}

Institut für Psychosoziale Medizin und

Psychotherapie,

Universitätsklinikum Jena, Friedrich-Schiller-

Universität Jena

Stoystr. 3, 07740 Jena

lisa.janetzki@uni-jena.de

\section{Einhaltung ethischer Richtlinien}

Interessenkonflikt. L. Janetzki, A. Gussew,

R. Malessa, U. Habenicht, J.R. Reichenbach, B. Strauß und $C$. Borys geben an, dass kein Interessenkonflikt besteht. Die Studie wurde unterstützt von der Berufsgenossenschaft Nahrungsmittel und Gastgewerbe.

Alle im vorliegenden Manuskript beschriebenen Untersuchungen am Menschen wurden mit Zustimmung der Ethik-Kommission der FriedrichSchiller-Universität Jena im Einklang mit nationalem Recht sowie gemäß der Deklaration von Helsinki von 1975 (in der aktuellen überarbeiteten Fassung von 2013) durchgeführt. Von allen beteiligten Probanden liegt eine Einverständniserklärung vor.

\section{Literatur}

1. Bhagwagar Z, Wylezinska $M$, Jezzard $P$ et al (2008) Low GABA concentrations in occipital cortex and anterior cingulate cortex in medicationfree, recovered depressed patients. Int J Neuropsychopharmacol 11:255-260

2. Bundesärztekammer (BÄK), Kassenärztliche Bundesvereinigung (KBV), Arbeitsgemeinschaft der Wissenschaftlichen Medizinischen Fachgesellschaften (AWMF). Nationale VersorgungsLeitlinie Kreuzschmerz - Langfassung. Letzte Überarbeitung 10/2015. http://www.leitlinien.de/nvl/kreuzschmerz. Zugegriffen: 5. Dez. 2015
3. Cauda F, Palermo S, Costa T et al (2014) Gray matter alterations in chronic pain: a networkoriented meta-analytic approach. Neuroimage Clinical 4:676-686

4. Choi DW (1992) Excitotoxic cell death. J Neurobiol 23:1261-1276

5. Cohen J (1988) Statistical power analysis for behavioral sciences. Erlbaum, Hillsdale

6. Foerster BR, Petrou M, Edden RA et al (2012) Reduced insular gamma-aminobutyric acid in fibromyalgia. Arthritis Rheum 64:579-583

7. Gabbay V, Mao X, Klein RG et al (2012) Anterior cingulate cortex gamma-aminobutyric acid in depressed adolescents: relationship to anhedonia. Arch Gen Psychiatry 69:139-149

8. Grachev ID, Fredrickson BE, Apkarian AV (2000) Abnormal brain chemistry in chronic back pain: an in vivo proton magnetic resonance spectroscopy study. Pain 89:7-18

9. Gussew A, Rzanny R, Erdtel M et al (2010) Timeresolved functional $1 \mathrm{H}$ MR spectroscopic detection of glutamate concentration changes in the brain during acute heat pain stimulation. Neuroimage 49:1895-1902

10. Gussew A, Rzanny R, Gullmar D et al (2011) 1H-MR spectroscopic detection of metabolic changes in pain processing brain regions in the presence of non-specific chronic low back pain. Neuroimage 54:1315-1323

11. Harris RE, Clauw DJ (2012) Imaging central neurochemical alterations in chronic pain with proton magnetic resonance spectroscopy. Neurosci Lett 520:192-196

12. Hasenbring $M$, Hallner D, Klasen B (2001) Psychologische Mechanismen im Prozess der Schmerzchronifizierung. Unter- oder überbewertet?. Schmerz 15:442-447

13. Latremoliere A, Woolf CJ (2009) Central sensitization: a generator of pain hypersensitivity by central neural plasticity. J Pain 10:895-926

14. Linton SJ (2000) A review of psychological risk factors in back and neck pain. Spine 25:1148-1156

15. Melzack R (2001) Pain and the neuromatrix in the brain. J Dent Educ 65:1378-1382

16. Mescher M, Merkle H, Kirsch J et al (1998) Simultaneous in vivo spectral editing and water suppression. NMR Biomed 11:266-272

17. Peyron R, Laurent B, Garcia-Larrea L (2000) Functional imaging of brain responses to pain. A review and meta-analysis (2000). Neurophysiol Clin 30:263-288

18. Pfingsten M, Nagel B, Emrich $O$ et al (2007) Deutscher Schmerzfragebogen. Handbuch. DGSS, Berlin

19. Robert Koch-Institut (2012) Rückenschmerzen. Gesundheitsberichterstattung des Bundes, Heft 53. Robert Koch-Institut, Berlin

20. Sanacora G, Treccani G, Popoli M (2012) Towards a glutamate hypothesis of depression. An emerging frontier of neuropsychopharmacology for mood disorders. Neuropharmacology 62:63-77

21. Sharma NK, McCarson K, Van Dillen L et al (2011) Primary somatosensory cortex in chronic low back pain - a H-MRS study. J Pain Res 4:143-150

22. Vartiainen N, Forss N (2014) [Imaging of brain changes in chronic pain]. Duodecim 130:15071514

23. Vellucci R (2012) Heterogeneity of chronic pain. Clin Drug Investig 32(Suppl 1):3-10

24. Wagner H, Puta C, Anders C et al (2009) Chronischer unspezifischer Rückenschmerz. Man Med 47:39-51 


\section{Diskussion}

\section{Inhaltliche Diskussion}

Das Ziel der Studie war es, Botenstoffe im Gehirn von Patienten mit chronischen Rückenschmerzen unter Berücksichtigung klinischer und psychologischer Merkmale zu untersuchen. Es wurden die Botenstoffe Glutamat $(\mathrm{Glx} / \mathrm{tCr})$ und GABA (GABA/tCr) sowie der Quotient Glutamat/GABA als Parameter für das Verhältnis von Erregung zu Hemmung in den Hirnregionen aCC und Insula gemessen. Die psychologischen Parameter Angst und Depressivität sowie die klinischen Parameter Schmerzdauer, Schmerzintensität und Chronifizierungsstadium wurden mit Fragebögen erhoben.

Schmerzpatienten und Kontrollgruppe wiesen im Gruppenvergleich in keiner Hirnregion signifikante Unterschiede der $\mathrm{Glx} / \mathrm{tCr}, \mathrm{GABA} / \mathrm{tCr}$ sowie Glx/GABA Quotienten auf. Der Quotient aus Glutamat/GABA war im aCC von Schmerzpatienten tendenziell höher als bei parallelisierten Kontrollpersonen. Dies könnte für einen Überschuss erregender Neurotransmitter bei chronischen Schmerzpatienten sprechen. Im Sinne der ,zentralen Sensibilisierung“ kann das Ergebnis als Ausdruck einer erhöhten Membranerregbarkeit und dysfunktionalen Erregungsaktivität interpretiert werden. Über diesen Mechanismus kann eine erhöhte Schmerzempfindlichkeit erklärt werden. Besonders in der Patientengruppe wiesen die mittels ${ }^{1}$ H-MRS gemessenen Neurotransmitter jedoch eine starke Varianz auf. Eine Erklärung hierfür findet sich zum Beispiel unter Berücksichtigung der klinischen Merkmale. Bezüglich Schmerzdauer und Schmerzintensität lagen bei den Patienten sehr unterschiedliche Ausprägungen vor. Ein Vergleich von Schmerzpatienten und Kontrollpersonen ist also dadurch erschwert, dass Schmerzpatienten keine homogene Gruppe darstellen. Rückenschmerzen sind ein multifaktorielles Problem. Dies spiegelt sich in der Streuung neurochemischer Veränderungen im zentralen Nervensystem wider.

Zum Verständnis der Ergebnisse ist auch eine Berücksichtigung der spezifischen Wirkungen der Neurotransmitter erforderlich. Wenn man nach aktuellem Forschungsstand davon ausgeht, dass bei Schmerzchronifizierung ein Überschuss erregender Neurotransmitter vorliegt, so bedeutet das, dass im Gehirn von Schmerzpatienten hohe Konzentrationen von Glutamat im synaptischen Spalt agieren (Foerster et al. 2012, Harris und Clauw 2012). Glutamat hat in hohen Konzentrationen einen exzitotoxischen Effekt: die Zellerregung führt durch einen massiven Kalziumeinstrom zum Zelltod und damit zum Verlust glutamaterger Neurone (Choi 1992, Gussew et al. 2011). Von welchen spezifischen Faktoren die Entgleisung des GABAGlutamat-Gleichgewichtes abhängt, ist bislang nicht geklärt. 
Im Folgenden soll diskutiert werden, welche psychologischen und klinischen Faktoren im Zusammenhang mit neurochemischen Veränderungen stehen.

Insgesamt zeigte die Patientengruppe im Vergleich zur Kontrollgruppe eine signifikant höhere Ausprägung der Merkmale Depressivität und Angst, die jedoch durchschnittlich unter dem klinischen Cut-Off $(\geq 12)$ lagen. Diese Befunde stimmen weitgehend mit früheren Studien überein, die Komorbiditäten und Lebensqualität von Rückenschmerzpatienten untersuchten (Bair et al. 2003, Castro et al. 2009, Busch et al. 2013, Lame et al. 2005, Harter et al. 2003). Akute Depressionen waren Ausschlusskriterium der Studie und wurden während der Patientenrekrutierung mittels SKID ausgeschlossen. Die vorliegenden subklinischen Ausprägungen für Depressivität und Angst standen im Zusammenhang mit den Ausprägungen von GABA und Glutamat im aCC. In Übereinstimmung mit der aktuellen Studienlage wurde ein Zusammenhang zwischen niedrigen GABA/tCr Quotienten und erhöhter Depressivität festgestellt (Bhagwagar et al. 2008, Gabbay et al. 2012). Angst war ein Prädiktor für niedrige Glx/tCr Quotienten. Es konnte also gezeigt werden, dass die mittels psychologischer Tests ermittelten psychischen Merkmale auch neurochemische Korrelate aufweisen. Schmerzen und Depression stehen in einem engen Zusammenhang, wobei die Kausalität dieses Zusammenhanges weiterhin ungeklärt bleibt. In bisherigen Studien mit ${ }^{1} \mathrm{H}-\mathrm{MRS}$ wurden nur Schmerz- oder Depressionspatienten untersucht. Schmerzpatienten weisen jedoch häufig depressive Symptome auf, ohne das Vollbild einer Depression zu erfüllen. Es ist bekannt, dass diese psychologischen Merkmale für die Therapieplanung und das klinische Outcome relevant sind (Gatchel und Gardea 1999, van der Hulst et al. 2008). Die Frage, inwieweit die ermittelten Zusammenhänge geeignet sind um die Prognose und den Therapieerfolg von chronischen Schmerzpatienten vorherzusagen, bietet Spielraum für weitere Untersuchungen. Auffällig war zudem, dass in der Insula kein Zusammenhang zwischen psychischen Merkmalen und Neurotransmittern nachgewiesen wurde. Dies könnte ein Hinweis darauf sein, dass der aCC in erster Linie für die psychische Bewertung und Verarbeitung von Schmerzen verantwortlich ist. Belege dafür wurden auch schon in PET- und fMRT-Studien erbracht, die nachwiesen, dass die Aktivität im aCC im Wesentlichen von affektiven Begleitzuständen abhängig ist (Peyron et al. 2000, Ploner und Schnitzler 2004).

Eine weitere Fragestellung dieser Arbeit war es, den Zusammenhang zwischen klinischen Merkmalen und den Neurotransmittern GABA und Glutamat zu untersuchen. Die Merkmale Schmerzintensität, Schmerzdauer und Chronifizierungsstadium (MPSS) wurden ausgewählt, 
da sie sich in früheren Studien als prognostisch von größter Bedeutung erwiesen haben (Hasenbring 1998, Verkerk et al. 2012).

In unserer Patientengruppe zeigte sich in der Insula mit steigender Schmerzintensität eine Zunahme von $\mathrm{Glx} / \mathrm{tCr}$ sowie Abnahme von $\mathrm{GABA} / \mathrm{tCr}$. $\mathrm{Zu}$ einem ähnlichen Ergebnis kam eine Studie aus dem Jahr 2011, die Glutamat bisher nur im somatosensiblen Kortex von Rückenschmerzpatienten und gesunden Kontrollpersonen verglich (Sharma et al. 2011). Auch hier wurde kein signifikanter Unterschied zwischen den Gruppen, jedoch ein Zusammenhang von Schmerzintensität und erhöhtem Glutamatlevel nachgewiesen. Dies spricht für einen zunehmenden Überschuss von erregenden Neurotransmittern in Abhängigkeit von der durchschnittlichen Schmerzintensität. In unserer Studie wurde dieser Befund zum ersten Mal auch in der Insula nachgewiesen. Unter Anbetracht von Erkenntnissen aus fMRT-Studien lässt sich eine Beteiligung der Insula an sensorisch-diskriminativen Aspekten der Schmerzverarbeitung (Peyron et al. 2000, Bornhovd et al. 2002) ableiten.

Des Weiteren wurde in der Stichprobe der Schmerzpatienten das Chronifizierungsstadium nach MPSS erhoben. Die Heterogenität der Stichprobe spiegelte sich auch in der Verteilung der Chronifizierungsstadien wider. Die meisten Patienten wurden dem 2. Stadium der Chronifizierung zugeordnet, jedoch konnte auf Grund der Stichprobengröße keine Normalverteilung erreicht werden. Mit steigendem Chronifizierungsstadium zeigte sich eine Abnahme von Glx/tCr im aCC. Der Zusammenhang zwischen dem Stadium der Chronifizierung und der Ausprägung zentraler Botenstoffe ist besonders hervorzuheben: die Bedeutung des MPSS als prognostisch und für die Therapieplanung entscheidender Parameter ist bereits belegt (Hampel und Moergel 2009). Der parallel beobachtete Verlust des erregenden Neurotransmitters Glutamat wurde in unserer Studie erstmals nachgewiesen. Dieser Effekt könnte unter Berücksichtigung des oben beschriebenen Mechanismus für eine erhöhte Sensibilität der Zellen gegenüber der exzitotoxischen Wirkung Glutamats in höheren Chronifizierungsstadien sprechen. Bei geringer Chronifizierung steigt die Erregung in schmerzverarbeitenden Hirnregionen an. Bei hoher Chronifizierung liegt ein massiver Glutamatexzess vor und der zytotoxische Effekt überwiegt. Dieser Mechanismus könnte auch die, bei Schmerzpatienten nachgewiesenen, strukturellen Hirnveränderungen erklären. In mehreren Studien wurde eine Atrophie schmerzverarbeitender Hirnregionen, insbesondere des aCC beobachtet (May 2008, Ruscheweyh et al. 2011). Eine Verifizierung des Zusammenhanges zwischen $\mathrm{Glx} / \mathrm{tCr}$ und Chronifizierungsstadium an normalverteilten Stichproben ist erforderlich. Die Frage, ob der prognostische Wert des MPSS durch Glutamatwirkung vermittelt wird oder ob Glutamatveränderungen zusätzlich als Faktor für 
die Therapieplanung berücksichtigt werden sollten, könnte ein Ansatz für weitere Studien mit kontrollierter Beeinflussung glutamaterger Neurotransmission sein.

Die dem biopsychosozialen Modell der Schmerzchronifizierung zu Grunde liegende Annahme, dass biologische, psychologische und soziale Faktoren nicht unabhängig existieren, sondern in einer komplexen Interdependenz miteinander in Verbindung stehen, lässt sich auch neurochemisch belegen. Die Berücksichtigung von ${ }^{1} \mathrm{H}-\mathrm{MRS}$-Daten erweitert das Verständnis der Pathomechanismen der Schmerzchronifizierung. Um Schmerzpatienten erfolgreich zu therapieren und eine Chronifizierung $\mathrm{zu}$ verhindern, ist eine multimodale Diagnostik notwendig. Der ergänzende Wert der Erhebung neurochemischer Parameter ist im Weiteren zu prüfen.

\section{Methodische Diskussion}

In dieser Studie wurden mit ${ }^{1}$ H-MRS und SKID sowohl für die funktionelle Bildgebung als auch für die psychologische Diagnostik sehr aufwendige Messmethoden eingesetzt. Die Ergebnisse sind unter Berücksichtigung von methodischen Beschränkungen zu interpretieren. Vor allem aus der Stichprobengröße (N=19 je Gruppe) ergeben sich Einschränkungen bezüglich der Generalisierbarkeit der Ergebnisse. Bei einer kleinen Stichprobe ist die Wahrscheinlichkeit alle relevanten Unterschiede zwischen den Gruppen nachzuweisen, geringer als bei größeren Stichproben. Das Studiendesign umfasste eine sehr umfangreiche, zeitaufwändige Diagnostik (4 Stunden pro Studienteilnehmer). Aus diesem und auf Grund der Ausschlusskriterien konnte nur eine vergleichsweise kleine Stichprobe in die Studie eingeschlossen werden. Auf eine Analyse über Ausreißer wurde verzichtet. Auch war die Stichprobe bezüglich der Merkmale Chronifizierungsstadium und Schmerzintensität sehr heterogen und nicht normalverteilt. Die Stichprobengröße ist allerdings mit ähnlich angelegten Studien vergleichbar (Gussew et al. 2011, Sharma et al. 2011). Da insbesondere die ${ }^{1}$ H-MRS-Messung eine anspruchsvolle und neue Forschungsmethode darstellt, sind in diesem frühen Stadium Arbeiten zur Hypothesengenerierung explorativ an kleinen Stichproben vertretbar.

Des Weiteren ist die Generalisierbarkeit der Ergebnisse durch die Ausschlusskriterien eingeschränkt. Patienten, die aktuell psychisch erkrankt waren sowie Patienten mit regelmäßiger Opiatmedikation mussten wegen bekanntem Einfluss auf die Neurotransmitterkonzentrationen von der Studie ausgeschlossen werden und sind somit in den Ergebnissen nicht repräsentiert (Godlewska et al. 2014, Hermann et al. 2012). Die 
Durchführung eines querschnittlichen Studiendesigns erlaubt zudem keine Aussage über die Kausalität der gemessenen Zusammenhänge. Ob neurochemische Veränderungen Ursache oder Folge des Chronifizierungsprozesses sind, bleibt ungewiss und kann nur im Rahmen von Wiederholungsmessungen und Längsschnittstudien überprüft werden. Auf Grundlage des biopsychosozialen Modells der Schmerzchronifizierung ist am ehesten von einer komplexen Interdependenz aller auftretenden Faktoren auszugehen.

Neben dem Studiendesign und der Stichprobe müssen auch die verwendeten Messinstrumente kritisch hinterfragt werden. Die psychischen Merkmale Depressivität und Angst sowie die klinischen Merkmale Schmerzdauer und Schmerzintensität wurden mittels Fragebögen zur Selbstauskunft erhoben. Verzerrungen im Sinne von ,response bias“ können nicht ausgeschlossen werden. Der HADS-D wurde zur Erfragung von Depressivität und Angst genutzt. Der HADS weist für beide Subskalen eine gute Reliabilität ( $\alpha=0,8$ und Split-halfReliabilitäten $=0,8, \mathrm{rtt}>0.8$ ) auf und wurde in zahlreichen publizierten Studien validiert bzw. klinisch eingesetzt (C. Herrmann-Lingen 1995, Herrmann 1997). Das Chronifizierungsstadium wurde nach MPSS durch einen Interviewer ermittelt. Das MPSS gilt als valides Instrument zur Einteilung von Schmerzpatienten in Chronifizierungsstadien (Frettlöh et al. 2003). Die Quantifizierung von GABA und Glutamat erfolgte mittels ${ }^{1} \mathrm{H}-\mathrm{MRS}$. Trotz ihrer einmalig gegebenen Möglichkeit, Neurotransmitterkonzentrationen in vivo, nicht invasiv und ohne Einsatz ionisierender Strahlung messen zu können, weist die ${ }^{1} \mathrm{H}-\mathrm{MRS}$ eine relativ geringe spatiale Auflösung auf. Hierdurch können einerseits die Kontaminationen der Ergebnisse in untersuchten Hirnregionen durch Beiträge aus benachbarten anatomischen Arealen und, andererseits, die Effekte bedienerspezifischer Positionierung des Zielvolumens in der Zielregion sowie der anatomischen Variation auf gemessene Parameter nicht ausgeschlossen werden. Darüber hinaus lassen sich die spektroskopisch gemessenen Glutamatänderungen nicht eindeutig dem Neurotransmitterstoffwechsel und/oder den energiemetabolischen Prozessen zuweisen. Die Differenzierung dieser Prozesse sei daher künftigen Messungen bei höheren magnetischen Feldstärken (z.B. $\geq 7$ T) vorbehalten, die eine isolierte Quantifizierung von Glutamat und seinem bei der Neurotransmission aktiven Nebenprodukt Glutamin vorbehalten (Tkac et al. 2001).

Trotz dieser methodischen Einschränkungen konnten wertvolle Erkenntnisse bezüglich des Zusammenhanges psychischer, klinischer und neurochemischer Parameter bei Schmerzpatienten gewonnen und eine Basis für zukünftige Studien geschaffen werden. 


\section{Schlussfolgerung für die Forschung}

Sowohl klinische als auch psychische Merkmale von Patienten mit chronischen Rückenschmerzen stehen im Zusammenhang mit Änderungen von Neurotransmittern in den schmerzverarbeitenden Hirnregionen aCC und Insula. Das biopsychosoziale Modell der Schmerzchronifizierung wurde bereits 1976 entwickelt und seitdem in zahlreichen Studien belegt (Adler 2009, Engel 1977). Bislang liegen jedoch wenige Daten bezüglich der zentralen Verknüpfung von psychosozialen und biologischen Parametern vor. Eine fundierte Forschung ist jedoch notwendig, um den Prozess der Schmerzchronifizierung $\mathrm{zu}$ verstehen und therapeutisch eingreifen zu können.

Um die Repräsentativität zu erhöhten, sind Folgestudien an größeren Stichproben notwendig. Bei einem Vergleich mit Kontrollpersonen ist eine Parallelisierung der Gruppen über Alter und Geschlecht unbedingt notwendig, um diese Einflussfaktoren auszuschließen (Gao et al. 2013, Hadel et al. 2013). In dieser Studie wurde kein signifikanter Unterschied zwischen Patienten und Kontrollpersonen nachgewiesen, was in erster Linie auf die Heterogenität der Patientengruppe zurückgeführt wurde. Eine Herausforderung für künftige Studien wäre beispielsweise ein Vergleich der Neurotransmitter von Schmerzpatientengruppen unterteilt nach Chronifizierungsstadien. Das Studiendesign lässt sich auf weitere Merkmale von Schmerzpatienten ausweiten. Besonders interessant wäre hier der Zusammenhang zwischen Neurotransmittern und potenziell prognostisch und therapierelevanten Markern wie schmerzbezogene Kognitionen, Copingverhalten und interpersonellem Problemverhalten (Linton 2000). In Längsschnittstudien könnten Veränderungen von Neurotransmittern z.B. über den Verlauf einer multimodalen Schmerztherapie untersucht werden.

Aus messtechnischen Gründen wurden in dieser Studie nur die Hirnregionen aCC und Insula untersucht. In Folgestudien sollten zusätzlich Messungen in Kontrollregionen, die nicht schmerzverarbeitend sind, durchgeführt werden. Die Schmerzmatrix umfasst außerdem noch Thalamus, somatosensorischen und präfrontalen Kortex. Künftige Studien könnten sich mit der Frage beschäftigen, wie sich Neurotransmitter bei Schmerzpatienten in diesen Hirnregionen verhalten, ob es spezifische Zusammenhänge zwischen klinischen und psychischen Merkmalen gibt und ob Korrelationen zwischen Neurotransmittern innerhalb der Matrix bestehen. 


\section{Schlussfolgerung für die klinische Praxis}

Vor dem Hintergrund der Prävalenz von Rückenschmerzen in Deutschland und dem hohen Anteil therapierefraktärer Schmerzen ist eine Verbesserung der Therapieplanung unbedingt notwendig. Voraussetzung hierfür ist eine ausführliche Diagnostik und Berücksichtigung der individuellen medizinischen Befunde der Patienten zu einem möglichst frühen Zeitpunkt in der Krankheitsentwicklung, vor Beginn der Chronifizierung. Nach aktueller nationaler Versorgungsleitlinie für Kreuzschmerzen der Arbeitsgemeinschaft der Wissenschaftlichen Medizinischen Fachgesellschaften (AWMF) wird bereits bei therapieresistenten Schmerzen mit einer Dauer länger 4 Wochen eine Erhebung psychosozialer Risikofaktoren empfohlen. Die Berücksichtigung von Neurotransmitterveränderungen ermöglicht eine zusätzliche Charakterisierung der Patienten und schafft eine Verknüpfung zwischen psychischen und klinischen Befunden. Ihr Wert für die Vorhersage des Krankheitsverlaufes und Therapieansprechens ist noch zu prüfen.

Möglicherweise können neurochemische Befunde in Zukunft zur Optimierung der Versorgung von Schmerzpatienten genutzt werden. ${ }^{1} \mathrm{H}-\mathrm{MRS}$-Daten könnten als Parameter für ein Therapiemonitoring oder für Therapien mit Biofeedback hilfreich werden. Eine Studie an Patienten mit bipolarer Depression konnte bereits nachweisen, dass Veränderungen von Neurotransmittern mit Therapieerfolgen korrelieren (Machado-Vieira et al. 2015). Möglicherweise kann dieser Effekt auch in multimodalen Schmerztherapien genutzt werden. Des Weiteren belegen die gemessenen Zusammenhänge den Wert der Einteilung in Chronifizierungsstadien nach MPSS.

Die Evaluation von ${ }^{1} \mathrm{H}$-MRS-Daten für eine personalisierte analgetische Pharmakotherapie und die Entwicklung neuer Medikamente befindet sich noch in einem sehr frühen Stadium. Die schmerzreduzierende Wirkung von Medikamenten wie Pregabalin (NMDA-RezeptorAntagonist), die in die Wirkmechanismen von GABA und Glutamat eingreifen, konnte bereits in einigen Studien nachgewiesen werden (Harris et al. 2013, Giladi et al. 2015). Noch wird das Medikament jedoch „off label“, dass heißt zulassungsüberschreitend, verschrieben. $\mathrm{Ob}$ Patientengruppen mit hohen Glutamat- oder geringen GABA-Werten besonders gut auf die Wirkung ansprechen, wurde bis zum aktuellen Zeitpunkt noch nicht untersucht.

Insgesamt sind die diagnostischen, therapeutischen und pharmakologischen Konsequenzen, der mit ${ }^{1}$ H-MRS ermittelbaren Daten nicht vollständig absehbar. Die in vivo Messung von Neurotransmittern bietet sowohl für die Forschung als auch für die praktische Therapie von Schmerzpatienten ein großes Potenzial. Eine enge Zusammenarbeit eines interdisziplinäres 
Teams von Psychologen, Medizinern, Medizinphysikern und Neurowissenschaftlern ist in diesem Zusammenhang für die optimale Versorgung der Patienten von größter Bedeutung. 


\section{Literaturverzeichnis:}

Adler RH. 2009. Engel's biopsychosocial model is still relevant today. Journal of Psychosomatic Research, 67 (6):607-611.

Apkarian AV, Sosa Y, Sonty S, Levy RM, Harden RN, Parrish TB, Gitelman DR. 2004. Chronic back pain is associated with decreased prefrontal and thalamic gray matter density. Journal of Neuroscience, 24 (46):10410-10415.

Auer DP, Putz B, Kraft E, Lipinski B, Schill J, Holsboer F. 2000. Reduced glutamate in the anterior cingulate cortex in depression: an in vivo proton magnetic resonance spectroscopy study. Biol Psychiatry, 47 (4):305-313.

Bair MJ, Robinson RL, Katon W, Kroenke K. 2003. Depression and pain comorbidity: a literature review. Arch Intern Med, 163 (20):2433-2445.

Bhagwagar Z, Wylezinska M, Jezzard P, Evans J, Boorman E, P MM, P JC. 2008. Low GABA concentrations in occipital cortex and anterior cingulate cortex in medicationfree, recovered depressed patients. Int J Neuropsychopharmacol, 11 (2):255-260.

BKK D Gesundheitsreport 2013 http://www.bkk-dachverband.de/bkk-news/357-pmgesundheitsreport2013.

Bornhovd K, Quante M, Glauche V, Bromm B, Weiller C, Buchel C. 2002. Painful stimuli evoke different stimulus-response functions in the amygdala, prefrontal, insula and somatosensory cortex: a single-trial fMRI study. Brain, 125 (Pt 6):1326-1336.

Borys C, Lutz J, Strauss B, Altmann U. 2015. Effectiveness of a Multimodal Therapy for Patients with Chronic Low Back Pain Regarding Pre-Admission Healthcare Utilization. Plos One, 10 (11).

Borys C, Nodop S, Tutzschke R, Anders C, Scholle HC, Strauß B. 2013. Evaluation of the German new back school. Der Schmerz, 27 (6):588-596.

Buchner M, Neubauer E, Barie A, Schiltenwolf M. 2007. [Comorbidity in patients with chronic low back pain]. Schmerz, 21 (3):218-225.

Busch MA, Maske UE, Ryl L, Schlack R, Hapke U. 2013. Prevalence of depressive symptoms and diagnosed depression among adults in Germany. Bundesgesundheitsblatt - Gesundheitsforschung - Gesundheitsschutz, 56 (5):733-739.

C. Herrmann-Lingen UB, R. P. Snaith. 1995. Hospital Anxiety and Depression Scale -Deutsche Version. Verlag Hans Huber, Bern (C) (1995) 
Castro M, Kraychete D, Daltro C, Lopes J, Menezes R, Oliveira I. 2009. Comorbid anxiety and depression disorders in patients with chronic pain. Arq Neuropsiquiatr, 67 (4):982-985.

Choi CH, Coupland NJ, Bhardwaj PP, Kalra S, Casault CA, Reid K, Allen PS. 2006. T-2 measurement and quantification of glutamate in human brain in vivo. Magnetic Resonance in Medicine, 56 (5):971-977.

Choi DW. 1992. Excitotoxic cell death. J Neurobiol, 23 (9):1261-1276.

Cleve M, Gussew A, Reichenbach JR. 2015. In vivo detection of acute pain-induced changes of GABA plus and Glx in the human brain by using functional H-1 MEGAPRESS MR spectroscopy. Neuroimage, 105:67-75.

DAK-Gesundheit. 2014. DAK-Gesundheitsreport 2014, Analyse der Arbeitsunfähigkeitsdaten. Berlin.

Dunlop BW, Nemeroff CB. 2007. The role of dopamine in the pathophysiology of depression. Arch Gen Psychiatry, 64 (3):327-337.

Engel GL. 1977. The need for a new medical model: a challenge for biomedicine. Science, 196 (4286):129-136.

Fayed N, Garcia-Campayo J, Magallon R, Andres-Bergareche H, Luciano JV, Andres E, Beltran J. 2010. Localized 1H-NMR spectroscopy in patients with fibromyalgia: a controlled study of changes in cerebral glutamate/glutamine, inositol, choline, and N-acetylaspartate. Arthritis Res Ther, 12 (4):R134.

Foerster BR, Petrou M, Edden RA, Sundgren PC, Schmidt-Wilcke T, Lowe SE, Harte SE, Clauw DJ, Harris RE. 2012. Reduced insular gamma-aminobutyric acid in fibromyalgia. Arthritis Rheum, 64 (2):579-583.

Frettlöh J, Maier C, Gockel H, Hüppe M. 2003. Validität des Mainzer Stadienmodells der Schmerzchronifizierung bei unterschiedlichen Schmerzdiagnosen. Der Schmerz, 17 (4):240-251.

Frye MA, Watzl J, Banakar S, O'Neill J, Mintz J, Davanzo P, Fischer J, Chirichigno JW, Ventura J, Elman S, Tsuang J, Walot I, Thomas MA. 2007. Increased anterior cingulate/medial prefrontal cortical glutamate and creatine in bipolar depression. Neuropsychopharmacology, 32 (12):2490-2499.

Gabbay V, Mao X, Klein RG, Ely BA, Babb JS, Panzer AM, Alonso CM, Shungu DC. 2012. Anterior cingulate cortex gamma-aminobutyric acid in depressed adolescents: relationship to anhedonia. Arch Gen Psychiatry, 69 (2):139-149. 
Gao F, Edden RA, Li M, Puts NA, Wang G, Liu C, Zhao B, Wang H, Bai X, Zhao C, Wang X, Barker PB. 2013. Edited magnetic resonance spectroscopy detects an age-related decline in brain GABA levels. Neuroimage, 78:75-82.

Gatchel RJ, Gardea MA. 1999. Psychosocial issues - Their importance in predicting disability, response to treatment, and search for compensation. Neurologic Clinics, 17 (1):149-+.

Gatchel RJ, Peng YB, Peters ML, Fuchs PN, Turk DC. 2007. The biopsychosocial approach to chronic pain: scientific advances and future directions. Psychol Bull, 133 (4):581-624.

Giladi H, Choiniere M, Fitzcharles MA, Ware MA, Tan XM, Shir Y. 2015. Pregabalin for chronic pain: does one medication fit all? Current Medical Research and Opinion, 31 (7):1403-1411.

Godlewska BR, Near J, Cowen PJ. 2014. Neurochemistry of major depression: a study using magnetic resonance spectroscopy. Psychopharmacology (Berl).

Grachev ID, Fredrickson BE, Apkarian AV. 2000. Abnormal brain chemistry in chronic back pain: an in vivo proton magnetic resonance spectroscopy study. Pain, 89 (1):7-18.

Gussew A, Rzanny R, Gullmar D, Scholle HC, Reichenbach JR. 2011. 1H-MR spectroscopic detection of metabolic changes in pain processing brain regions in the presence of non-specific chronic low back pain. Neuroimage, 54 (2):1315-1323.

Gussew A, Rzanny R, Erdtel M, Scholle HC, Kaiser WA, Mentzel HJ, Reichenbach JR. 2010. Time-resolved functional 1H MR spectroscopic detection of glutamate concentration changes in the brain during acute heat pain stimulation. Neuroimage, 49 (2):1895-1902.

Hadel S, Wirth C, Rapp M, Gallinat J, Schubert F. 2013. Effects of age and sex on the concentrations of glutamate and glutamine in the human brain. J Magn Reson Imaging, 38 (6):1480-1487.

Hampel P, Moergel MF. 2009. [Staging of pain in patients with chronic low back pain in inpatient rehabilitation: validity of the Mainz Pain Staging System of pain chronification]. Schmerz, 23 (2):154-165.

Harris RE, Clauw DJ. 2012. Imaging central neurochemical alterations in chronic pain with proton magnetic resonance spectroscopy. Neurosci Lett, 520 (2):192-196. Harris RE, Napadow V, Huggins JP, Pauer L, Kim J, Hampson J, Sundgren PC, Foerster B, Petrou M, Schmidt-Wilcke T, Clauw DJ. 2013. Pregabalin rectifies aberrant brain 
chemistry, connectivity, and functional response in chronic pain patients. Anesthesiology, 119 (6):1453-1464.

Harter M, Weisser B, Reuter K, Bengel J. 2003. [Prevalence and risk factors of psychological burden and mental disorders in patients with musculoskeletal diseases -- a review of empirical studies]. Schmerz, 17 (1):50-59.

Hasenbring M. 1998. Predictors of efficacy in treatment of chronic low back pain. Curr Opin Anaesthesiol, 11 (5):553-558.

Hasenbring M, Hallner D, Klasen B. 2001. [Psychological mechanisms in the transition from acute to chronic pain: over- or underrated?]. Schmerz, 15 (6):442-447. Hasler G, van der Veen JW, Tumonis T, Meyers N, Shen J, Drevets WC. 2007. Reduced prefrontal glutamate/glutamine and gamma-aminobutyric acid levels in major depression determined using proton magnetic resonance spectroscopy. Archives of General Psychiatry, 64 (2):193-200.

Hermann D, Frischknecht U, Heinrich M, Hoerst M, Vollmert C, Vollstadt-Klein S, TuncSkarka N, Kiefer F, Mann K, Ende G. 2012. MR spectroscopy in opiate maintenance therapy: association of glutamate with the number of previous withdrawals in the anterior cingulate cortex. Addict Biol, 17 (3):659-667.

Herrmann C. 1997. International experiences with the Hospital Anxiety and Depression Scale--a review of validation data and clinical results. J Psychosom Res, 42 (1):1741.

Horng YS, Hwang YH, Wu HC, Liang HW, Mhe YJ, Twu FC, Wang JD. 2005. Predicting health-related quality of life in patients with low back pain. Spine (Phila Pa 1976), 30 (5):551-555.

Howells FM, Hattingh CJ, Syal S, Breet E, Stein DJ, Lochner C. 2014. H-magnetic resonance spectroscopy in social anxiety disorder. Prog Neuropsychopharmacol Biol Psychiatry.

IASP. 1986. Classification of chronic pain. Descriptions of chronic pain syndromes and definitions of pain terms. Prepared by the International Association for the Study of Pain, Subcommittee on Taxonomy. Pain Suppl, 3:S1-226.

Kröner-Herwig F, Klinger, Nilges. 2010. Schmerzpsychotherapie.

Lame IE, Peters ML, Vlaeyen JW, Kleef M, Patijn J. 2005. Quality of life in chronic pain is more associated with beliefs about pain, than with pain intensity. Eur J Pain, 9 (1):15-24. 
Latremoliere A, Woolf CJ. 2009. Central sensitization: a generator of pain hypersensitivity by central neural plasticity. J Pain, 10 (9):895-926.

Ling J, Campbell C, Heffernan TM, Greenough CG. 2007. Short-term prospective memory deficits in chronic back pain patients. Psychosom Med, 69 (2):144-148.

Linton SJ. 2000. A review of psychological risk factors in back and neck pain. Spine (Phila Pa 1976), 25 (9):1148-1156.

Machado-Vieira R, Gattaz WF, Zanetti MV, De Sousa RT, Carvalho AF, Soeiro-de-Souza MG, Leite CC, Otaduy MC. 2015. A Longitudinal (6-week) 3T (1)H-MRS Study on the Effects of Lithium Treatment on Anterior Cingulate Cortex Metabolites in Bipolar Depression. Eur Neuropsychopharmacol, 25 (12):2311-2317.

Mahmutyazicioglu K, Konuk N, Ozdemir H, Atasoy N, Atik L, Gundogdu S. 2005. Evaluation of the hippocampus and the anterior cingulate gyrus by proton MR spectroscopy in patients with post-traumatic stress disorder. Diagn Interv Radiol, $11(3): 125-129$.

Mao C, Wei L, Zhang Q, Liao X, Yang X, Zhang M. 2013. Differences in brain structure in patients with distinct sites of chronic pain: A voxel-based morphometric analysis. Neural Regen Res, 8 (32):2981-2990.

May A. 2008. Chronic pain may change the structure of the brain. Pain, 137 (1):7-15. McCracken LM, Turk DC. 2002. Behavioral and cognitive-behavioral treatment for chronic pain: outcome, predictors of outcome, and treatment process. Spine (Phila Pa 1976), 27 (22):2564-2573.

Melzack R. 2001. Pain and the neuromatrix in the brain. J Dent Educ, 65 (12):1378-1382.

Michael N, Erfurth A, Ohrmann P, Gossling M, Arolt V, Heindel W, Pfleiderer B. 2003. Acute mania is accompanied by elevated glutamate/glutamine levels within the left dorsolateral prefrontal cortex. Psychopharmacology, 168 (3):344-346.

Modi S, Rana P, Kaur P, Rani N, Khushu S. 2014. Glutamate level in anterior cingulate predicts anxiety in healthy humans: a magnetic resonance spectroscopy study. Psychiatry Res, 224 (1):34-41.

Mohler H. 2006. GABA(A) receptor diversity and pharmacology. Cell Tissue Res, 326 (2):505-516.

Nötzel D. 2011. Klassifikation von Rückenschmerz auf Basis elektrophysiologischer und psychosozialer Parameter. Jena: Bussert \& Stadeler. 
Orrego F, Villanueva S. 1993. The Chemical Nature of the Main Central Excitatory Transmitter - a Critical-Appraisal Based Upon Release Studies and Synaptic Vesicle Localization. Neuroscience, 56 (3):539-555.

Peyron R, Laurent B, Garcia-Larrea L. 2000. Functional imaging of brain responses to pain. A review and meta-analysis (2000). Neurophysiol Clin, 30 (5):263-288.

Pfingsten M, Schops P. 2004. Low back pain - From symptom to chronic disease. Zeitschrift Fur Orthopadie Und Ihre Grenzgebiete, 142 (2):146-152.

Ploner M, Schnitzler A. 2004. Cortical representation of pain. Der Nervenarzt, 75 (10):962-969.

Rae CD. 2014. A guide to the metabolic pathways and function of metabolites observed in human brain 1H magnetic resonance spectra. Neurochem Res, 39 (1):1-36.

Reme SE, Tangen T, Moe T, Eriksen HR. 2011. Prevalence of psychiatric disorders in sick listed chronic low back pain patients. Eur J Pain, 15 (10):1075-1080.

Ruscheweyh R, Deppe M, Lohmann H, Stehling C, Floel A, Ringelstein EB, Knecht S. 2011. Pain is associated with regional grey matter reduction in the general population. Pain, 152 (4):904-911.

Sanacora G, Treccani G, Popoli M. 2012. Towards a glutamate hypothesis of depression An emerging frontier of neuropsychopharmacology for mood disorders. Neuropharmacology, 62 (1):63-77.

Schmidt CO, Raspe H, Pfingsten M, Hasenbring M, Basler HD, Eich W, Kohlmann T. 2007. Back pain in the German adult population: prevalence, severity, and sociodemographic correlates in a multiregional survey. Spine (Phila Pa 1976), 32 (18):2005-2011.

Sezgin M, Hasanefendioglu EZ, Ali Sungur M, Incel NA, Cimen O, Kanik A, Shin G. 2014. Sleep quality in patients with chronic low back pain: A cross-sectional study assesing its relations with pain, functional status and quality of life. J Back Musculoskelet Rehabil.

Sharma NK, McCarson K, Van Dillen L, Lentz A, Khan T, Cirstea CM. 2011. Primary somatosensory cortex in chronic low back pain - a H-MRS study. J Pain Res, 4:143-150.

Shekelle PG, Markovich M, Louie R. 1995. An epidemiologic study of episodes of back pain care. Spine (Phila Pa 1976), 20 (15):1668-1673.

Somborski K, Bingel U. 2010. Functional imaging in pain research. Schmerz, 24 (4):385398. 
Statistisches-Bundesamt Krankheitskosten in Mio. € in Deutschland.

Techniker-Krankenkassen Gesundheitsreport 2013. Berufstätigkeit, Ausbildung und Gesundheit

Thomas Karov RL-R. 2013. Pharmakologie und Toxikologie. Köln: Dr. med Thomas Karov.

Tkac I, Andersen P, Adriany G, Merkle H, Ugurbil K, Gruetter R. 2001. In vivo 1H NMR spectroscopy of the human brain at 7 T. Magn Reson Med, 46 (3):451-456.

Tutzschke R, Anders C, Borys C, Nodop S, Rößler O, Strauß B, Scholle HC. 2014. Evaluation of the German new back school. Der Schmerz, 28 (2):166-174.

Valdes M, Collado A, Bargallo N, Vazquez M, Rami L, Gomez E, Salamero M. 2010. Increased glutamate/glutamine compounds in the brains of patients with fibromyalgia: a magnetic resonance spectroscopy study. Arthritis Rheum, 62 (6):1829-1836.

van der Hulst M, Vollenbroek-Hutten MM, Groothuis-Oudshoorn KG, Hermens HJ. 2008. Multidisciplinary rehabilitation treatment of patients with chronic low back pain: a prognostic model for its outcome. Clin J Pain, 24 (5):421-430.

Verkerk K, Luijsterburg PA, Miedema HS, Pool-Goudzwaard A, Koes BW. 2012.

Prognostic factors for recovery in chronic nonspecific low back pain: a systematic review. Phys Ther, 92 (9):1093-1108. 


\section{Danksagung}

Ich möchte mich bei Herrn Prof. Dr. phil. Bernhard Strauß für die Überlassung des Themas und die Unterstützung meiner Arbeit bedanken. Besonderer Dank gilt Frau Dr. Constanze Borys für die Betreuung der Arbeit.

Ich bedanke mich herzlich bei allen Patienten und Kontrollpersonen, die sich bereit erklärt haben, an der Studie teilzunehmen.

Danken möchte ich auch allen anderen Personen, die mich bei meiner Arbeit unterstützt haben, insbesondere Dr. Alexander Gussew.

Ein persönlicher Dank gilt außerdem meinem Freund Stefan Landmann, meiner Familie und meinen Freunden für ihre Unterstützung und Geduld. 


\section{Ehrenwörtliche Erklärung}

Hiermit erkläre ich, dass mir die Promotionsordnung der Medizinischen Fakultät der Friedrich-Schiller-Universität bekannt ist,

ich die Dissertation selbst angefertigt habe und alle von mir benutzten Hilfsmittel, persönlichen Mitteilungen und Quellen in meiner Arbeit angegeben sind,

mich folgende Personen bei der Auswahl und Auswertung des Materials sowie bei der Herstellung des Manuskripts unterstützt haben: Dr. Constanze Borys, Dr. Alexander Gussew, Prof. Dr. Bernhard Strauß, Dr. Uwe Altmann,

die Hilfe eines Promotionsberaters nicht in Anspruch genommen wurde und dass Dritte weder unmittelbar noch mittelbar geldwerte Leistungen von mir für Arbeiten erhalten haben, die im Zusammenhang mit dem Inhalt der vorgelegten Dissertation stehen,

dass ich die Dissertation noch nicht als Prüfungsarbeit für eine staatliche oder andere wissenschaftliche Prüfung eingereicht habe und

dass ich die gleiche, eine in wesentlichen Teilen ähnliche oder eine andere Abhandlung nicht bei einer anderen Hochschule als Dissertation eingereicht habe.

Ort, Datum

Unterschrift der Verfasserin 


\section{Lebenslauf}

\section{Persönliche Daten}

Name

Anschrift

Geburtsdatum

Geburtsort

Staatsangehörigkeit

Familienstand

Telefonnummer

E-Mail

\section{Ausbildung}

1997-2001

2001-2010

Mai 2001

Seit Oktober 2010

September 2012
Lisa Janetzki

Magdelstieg 19

07745 Jena

06.09 .1990

Köln

deutsch

ledig

015739632086

lisa.janetzki@uni-jena.de

Remigiusgrundschule in Bergheim

Erftgymnasium in Bergheim

Abitur mit Notendurchschnitt 1,2

Medizinstudium an der

Friedrich Schiller Universität in Jena

Erfolgreicher Abschluss des ersten Abschnitts

der Ärztlichen Prüfung mit Notendurchschnitt 2,0

\section{Weitere Ausbildungen und Tätigkeiten}

2012 - 2016: Studentische Hilfskraft am Institut für Anatomie II als Tutorin im Histologiekurs WS 2014: Studentische Hilfskraft an der Klinik für Neurologie des UKJ als Tutorin

Juli 2015: Teilnahme an der Sommerakademie für Integrative Medizin der Universität Witten-Herdecke

Mai - Juni 2016: Fünfwöchiges Volontariat bei NGO Taxi Peru im Krankenhausprojekt in Cusco (Peru)

\section{Besondere Kenntnisse}

Sprachen:

Englisch

Französisch

Spanisch 


\section{Posterbeiträge und Publikationen}

L. Janetzki, A. Gussew, R. Malessa, U. Habenicht, J.R. Reichenbach, B. Strauß, C. Borys. Hirnmetabolische Veränderungen bei chronischem Rückenschmerz. Schmerz: 1-7. Published ahead of print. DOI 10.1007/s00482-015-0082-5

Posterbeiträge bei

Deutscher Schmerzkongress, Hamburg, 2014

Symposium zur Prävention arbeitsbedingter Gesundheitsgefahren und Erkrankungen, Erfurt, 2014

Deutscher Kongress für Psychosomatische Medizin und Psychotherapie, Berlin, 2015 


\title{
Anhang
}

\section{A Ethikvotum}

\author{
Universitätsklinikum \\ Ethik-Kommission \\ Universitätsklinikum Jena - Ethik-Kommission - Postfach - 07740 Jena \\ Vorsitzende: Prof. Dr. med. Dagmar Barz \\ Dr. A. Gussew \\ IDIR I \\ Geschäftsstelle: Dr. phil. Ulrike Skorsetz \\ AG Medizinische Physik \\ Bachstraße 18 \\ 07743 Jena \\ 07740 Jena \\ Telefon 03641933770 \\ Telefax 03641933771 \\ E-Mail: ethikkommission@med.uni-jena.de \\ 25. Juli 2013

\section{Bearbeitungs-Nr. 1861-09/06} \\ 1H-Magnetresonanzspektosopische Untersuchungen zur Nachweisbarkeit biochemischer \\ "Veränderungen im zentralen und peripheren Nervensystem unter Einfluss von Schmerz"
}

\section{Sehr geehrter Dr. Gussew,}

hiermit bestätigen wir den Eingang und die Kenntnisnahme des Schreibens zur o. g. Studie vom 19. Juli dieses Jahres sowie der überarbeiteten Patienteninformation und Einwilligungserklärung.

Die Erweiterung der Studie um 25 Patienten und 25 Kontroll-Probanden, die mit neuartiger $1 \mathrm{H}-M R$ spektroskopische Technik (MEGAPRESS Methode) untersucht werden sollen sowie die Einführung eines standardisierten psychodiagnostischen Interviews zur Erfassung der psychischen Komorbidität und die Verwendung eines Fragebogens zu psychosozialen Merkmalen, Schmerzcharakteristika und Versorgungsmerkmalen werden zustimmend bewertet.

Die aktualisierte Patienteninformation und Einwilligungserklärung werden ebenfalls zustimmend bewertet.

Wir wünschen Ihnen weiterhin viel Erfolg!

Mit freundlichem Gruß

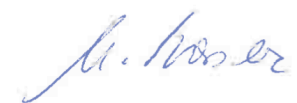

Prof. Dr. med. D. Barz

Vorsitzende der Ethik-Kommission

(i. A. Dr. U. Skorsetz)

Bachstraße 18. 07743 Jena Telefon 036419300

Internet: mmw uniklinikum-iena de

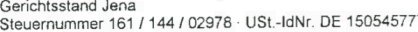
Bankverbindung: 2144 / 23053030 . Konto 221
Universitätsklinikum Jena . Körperschaft des öffentlichen Rechts als Teilkörperschaft der Friedrich-Schiller-Universitäta Jena Medwaltungstatsvorsitze

und Sprecher des Klinikumsvorstandes: Prof. Dr. Klaus Höfken Wissenschaftlicher Vorstand Prof. Dr. Klaus Benndorf 


\section{B Aufklärungsbogen für Patienten}

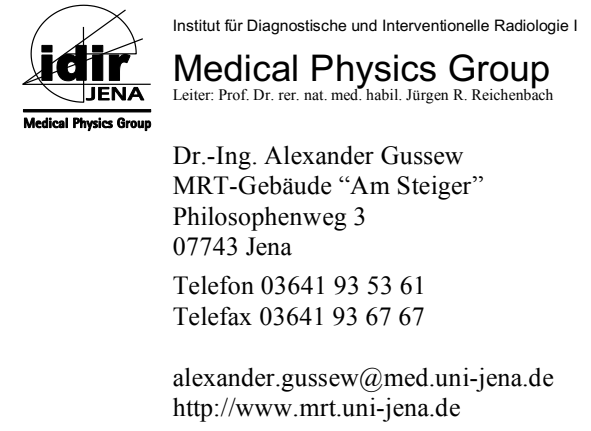

\section{Liebe Patientin, lieber Patient,}

Mit Hilfe der Protonen-Magnet-Resonanz-Spektroskopie ( $\left.{ }^{1} \mathrm{H}-\mathrm{MRS}\right)$ lassen sich biochemische Prozesse in vivo, das heißt im lebenden Körpergewebe nicht invasiv und ohne Röntgenstrahlung untersuchen. Damit konnten in der Vergangenheit viele physiologische und pathologische Prozesse umfassender aufgeklärt werden. Das Ziel unserer Studie besteht darin, mit Hilfe der ${ }^{1} \mathrm{H}-\mathrm{MRS}$ biochemische Änderungen im zentralen Nervensystem infolge chronischer Rückenschmerzen nachzuweisen. Damit bestünde die Möglichkeit, mehr über die Entstehung und Chronifizierung von Rückenschmerzen zu erfahren. Um andere mögliche Ursachen der biochemischen Änderungen auszuschließen, werden zusätzlich zur Untersuchung im MRT ein diagnostisches Interview und eine schriftliche Befragung durchgeführt. Die Dauer des Interviews beträgt ca. 90 min.

Die geplanten Untersuchungen im MRT verlaufen ohne Applikation pharmazeutischer Präparate, so dass für Sie dort die einzige Belastung in der relativ langen Untersuchungsdauer von max. 90 min. liegt. Während dieser Zeit sollte der Kopf nicht bewegt werden, da sonst das Ergebnis der gesamten Untersuchung in Frage gestellt wird.

Ihre Teilnahme an der Untersuchung erfolgt selbstverständlich freiwillig. Sie können die Untersuchung jederzeit, ohne Angaben von Gründen und ohne Folgen abbrechen. Alle Untersuchungsdaten werden von Ihren persönlichen Daten getrennt ausgewertet. Weder Firmen noch Behörden erhalten Einblick in Ihre persönlichen Daten und Untersuchungsbefunde. Lediglich das Gesamtresultat der Untersuchung wird (ohne Namensnennung) in wissenschaftlichen Zeitschriften und auf Fachtagungen veröffentlicht. Die Vorschriften über die ärztliche Schweigepflicht und den Datenschutz werden gewahrt. Es werden lediglich anonymisierte Daten ohne Namensnennungen weitergegeben. Während der Untersuchung (auf Klinikumsgelände) besteht ein Versicherungsschutz über die allgemeine Haftpflichtversicherung des Universitätsklinikums Jena. Falls Sie sich zu irgendeinem Zeitpunkt der Studie unwohl fühlen oder die Untersuchung abbrechen möchten, bitten wir Sie, dies umgehend einem unserer Mitarbeiter mitzuteilen.

Für weitere Fragen stehen wir Ihnen jederzeit gerne zur Verfügung. Bitte lesen sie noch den beigelegten allgemeinen Aufklärungsbogen zur MRT und unterschreiben Sie beide bei Einwilligung.

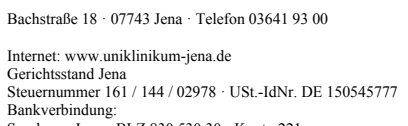

Universitätsklinikum Jena $\cdot$ Körperschaft des öffentlichen Rech als Teilkörperschaft der Friedrich-Schiller-Universität J Medizinischer Vorstand und Sprecher des Klinikumsvorstands: Prof. Dr. Klaus Höffken Wissenschaftlicher Vorstand: Prof. Dr. Klaus Benndorf 


\section{Universitätsklinikum}

Jena

\section{Patientenaufklärung für die Untersuchung}

${ }^{1}$ H-Magnetresonanzspektroskopische Untersuchungen zur Nachweisbarkeit biochemischer Veränderungen im zentralen Nervensystem unter dem Einfluss von chronischen Rückenschmerzen

Ich,

(Name des Studienteilnehmers)

habe diesen Aufklärungsbogen erhalten und gelesen und bin bereit, freiwillig an dieser Untersuchung teilzunehmen. Ich wurde darüber aufgeklärt, dass ich jederzeit, ohne Angaben von Gründen und ohne Folgen die Untersuchung abbrechen kann, und dass die im Rahmen dieser Untersuchung erhobenen Daten in pseudonymisiert Form wissenschaftlich ausgewertet werden.

Im Falle eines zufälligen pathologischen Befundes möchte ich informiert / nicht informiert werden.

(Nicht zutreffendes, bitte, durchstreichen.)

(Datum)

(Unterschrift PatientIn/ bzw. gesetzl. Vertreter)

Ich versichere, dass ich der betreffenden Person die Art und den Zweck der Untersuchung erklärt habe.

(Datum)

(Unterschrift aufklärender Arzt) 


\section{Aufklärungsbogen für Kontrollpersonen}

Universitätsklinikum Jena $\cdot$ Postfach $\cdot 07740$ Jena

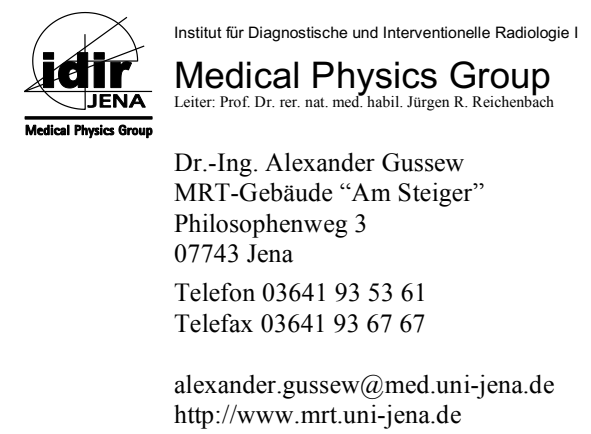

\section{Liebe Probandin, lieber Proband,}

Mit Hilfe der Protonen-Magnet-Resonanz-Spektroskopie ( ${ }^{1} \mathrm{H}-\mathrm{MRS}$ ) lassen sich biochemische Prozesse in vivo, das heißt im lebenden Körpergewebe nicht invasiv und ohne Röntgenstrahlung untersuchen. Damit konnten in der Vergangenheit viele physiologische und pathologische Prozesse umfassender aufgeklärt werden. Das Ziel unserer Studie besteht darin, mit Hilfe der ${ }^{1}$ H-MRS biochemische Änderungen im zentralen Nervensystem infolge chronischer Rückenschmerzen nachzuweisen. Damit bestünde die Möglichkeit, mehr über die Entstehung und Chronifizierung von Rückenschmerzen zu erfahren. Um andere mögliche Ursachen der biochemischen Änderungen auszuschließen, werden zusätzlich zur Untersuchung im MRT ein diagnostisches Interview und eine schriftliche Befragung durchgeführt. Die Dauer des Interviews beträgt ca. 90 min.

Die geplanten Untersuchungen im MRT verlaufen ohne Applikation pharmazeutischer Präparate, so dass für Sie dort die einzige Belastung in der relativ langen Untersuchungsdauer von max. 90 min. liegt. Während dieser Zeit sollte der Kopf nicht bewegt werden, da sonst das Ergebnis der gesamten Untersuchung in Frage gestellt wird.

Ihre Teilnahme an der Untersuchung erfolgt selbstverständlich freiwillig. Sie können die Untersuchung jederzeit, ohne Angaben von Gründen und ohne Folgen abbrechen. Alle Untersuchungsdaten werden von Ihren persönlichen Daten getrennt ausgewertet. Weder Firmen noch Behörden erhalten Einblick in Ihre persönlichen Daten und Untersuchungsbefunde. Lediglich das Gesamtresultat der Untersuchung wird (ohne Namensnennung) in wissenschaftlichen Zeitschriften und auf Fachtagungen veröffentlicht. Die Vorschriften über die ärztliche Schweigepflicht und den Datenschutz werden gewahrt. Es werden lediglich anonymisierte Daten ohne Namensnennungen weitergegeben. Während der Untersuchung (auf Klinikumsgelände) besteht ein Versicherungsschutz über die allgemeine Haftpflichtversicherung des Universitätsklinikums Jena. Falls Sie sich zu irgendeinem Zeitpunkt der Studie unwohl fühlen oder die Untersuchung abbrechen möchten, bitten wir Sie, dies umgehend einem unserer Mitarbeiter mitzuteilen.

Für weitere Fragen stehen wir Ihnen jederzeit gerne zur Verfügung. Bitte lesen sie noch den beigelegten allgemeinen Aufklärungsbogen zur MRT und unterschreiben Sie beide bei Einwilligung.

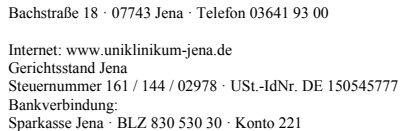

Sparkasse Jena · BLZ $83053030 \cdot$ Konto 22 
Universitätsklinikum Jena

\section{Probandenaufklärung für die Untersuchung}

${ }^{1}$ H-Magnetresonanzspektroskopische Untersuchungen zur Nachweisbarkeit biochemischer Veränderungen im zentralen Nervensystem unter dem Einfluss von chronischen Rückenschmerzen

Ich,

(Name des Studienteilnehmers)

habe diesen Aufklärungsbogen erhalten und gelesen und bin bereit, freiwillig an dieser Untersuchung teilzunehmen. Ich wurde darüber aufgeklärt, dass ich jederzeit, ohne Angaben von Gründen und ohne Folgen die Untersuchung abbrechen kann, und dass die im Rahmen dieser Untersuchung erhobenen Daten in pseudonymisiert Form wissenschaftlich ausgewertet werden.

Im Falle eines zufälligen pathologischen Befundes möchte ich informiert / nicht informiert werden.

(Nicht zutreffendes, bitte, durchstreichen.)

(Datum)

(Unterschrift ProbandIn/ bzw. gesetzl. Vertreter)

Ich versichere, dass ich der betreffenden Person die Art und den Zweck der Untersuchung erklärt habe.

(Datum)

(Unterschrift aufklärender Arzt) 
D Fragebogen für Patienten

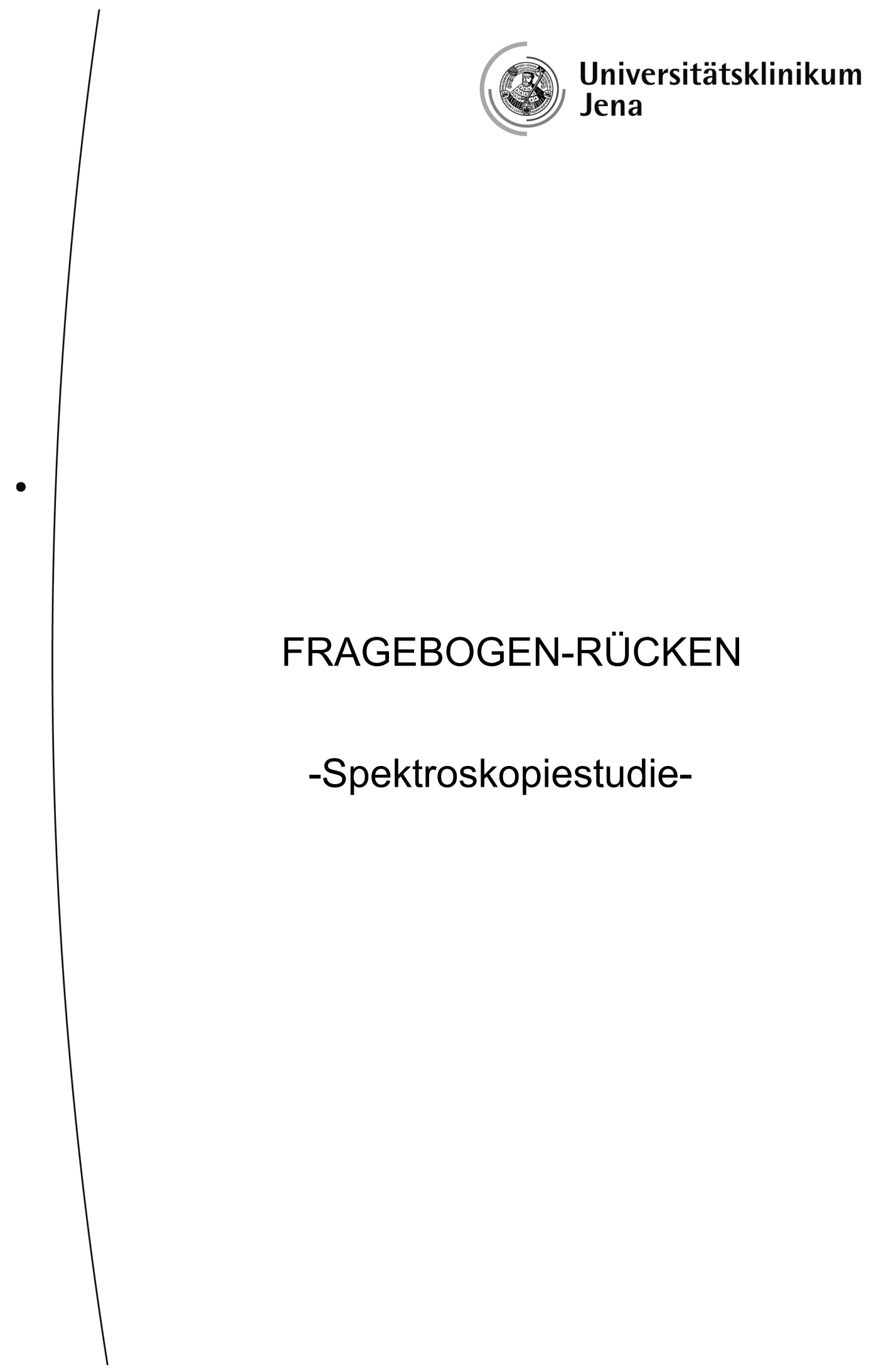




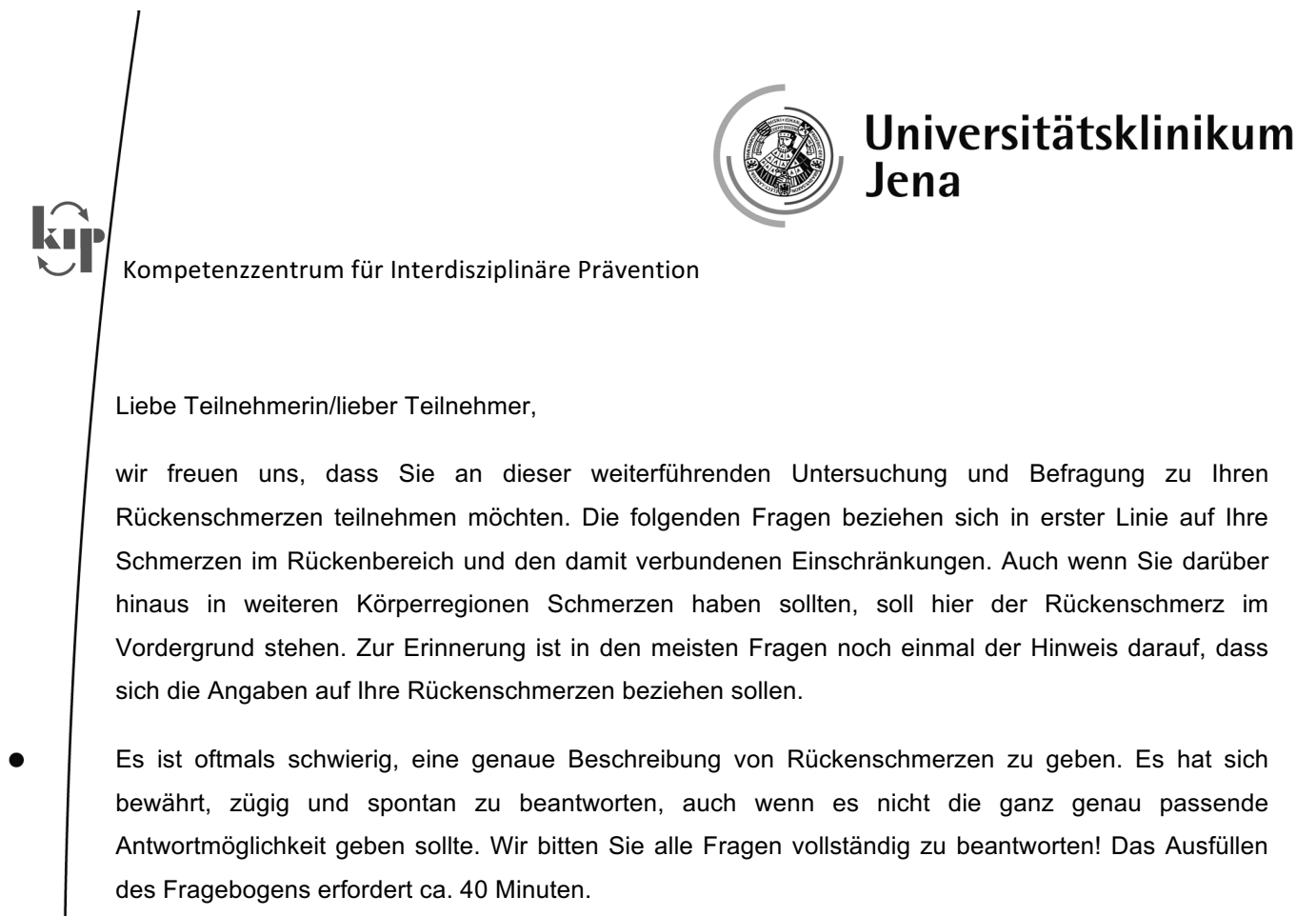

Sie können den Fragebogen sofort ausfüllen und dem Untersuchungsteam übergeben oder uns den ausgefüllten Bogen im vorbereiteten Rückumschlag (ohne Briefmarke) zusenden.

Bei Rückfragen können Sie sich per e-mail unter: Constanze.Borys@med.uni-jena.de melden.

Für die wissenschaftliche Auswertung des Fragebogens und des Interviews werden Ihre Daten pseudonymisiert verwertet, d.h. sie werden unter einer numerischen Codierung ohne Ihren Namen gespeichert und analysiert.

Wir bitten Sie hiermit um Ihr Einverständnis zur Abspeicherung der Daten. Dieses Einverständnis können Sie jeder Zeit widerrufen.

\section{Einverständniserklärung}

Ich bin mit der Abspeicherung und der pseudonymisierten Verwendung der im Fragebogen und Interview erhobenen Daten einverstanden. 


\section{Soziodemographie:}

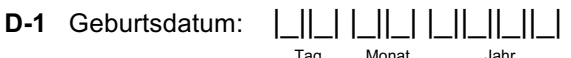

D-2. Geschlecht: $\quad$ weiblich $_{1} \square \quad$ männlich $_{0}$

D-3. Körpergröße (cm): | ______ Körpergewicht $(\mathrm{kg}):$ | _

D-4. Personenstand: $\quad$ ledig $\square \quad$ verheiratet $\square$ verwitwet $\square$ getrennt lebend $\square$ geschieden $\square$
D-5. Haben Sie Kinder?
$\square$ ja
$\square$ nein
Anzahl

D-6. Wer lebt gemeinsam mit Ihnen in Ihrem Haushalt? (Mehrfachantworten sind möglich):
ich lebe allein $\square$
Ehepartner/Partner $\square$
Kinder
(Schwieger-)Eltern $\square$

D-7. Welchen höchsten Schulabschluss haben Sie?

keinen Abschluss ${ }_{0} \square \quad$ Hauptschule / Volksschule 0 Realschule/Mittlere Reife/POS $\square$

Fachhochschulreife $_{2} \square \quad$ Abitur/allgemeine Hochschulreife ${ }_{3} \square$

D-8. Welche höchsten Berufsabschluss haben Sie?
keinen Abschluss $\square$
Berufsschule / Lehre $\square$
Fach- / Meisterschule
Fachhochschule $_{3}$
Postgraduiert (Dr.)
Universitätsabschluss

D-9. Welchen Beruf haben Sie erlernt?

D-10. Welche Tätigkeit üben Sie derzeit aus?

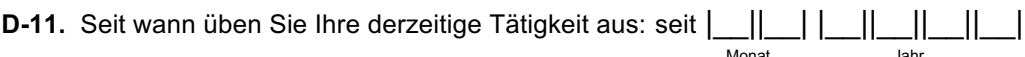

\section{Rückenschmerz}

In welcher Form traten Ihre Rückenschmerzen in den letzten 12 Monaten auf?

Zutreffendes bitte ankreuzen

Einmalige Rückenschmerzepisode, die weniger als 3 Monate (90 Tage) andauerte

Mehrmalige Rückenschmerzepisoden, die weniger als 3 Monate (90 Tage) andauerten

Mindestens 3 Monate Rückenbeschwerden ohne Unterbrechung (min. 90 aufeinander folgende Tage)

Wann genau haben Ihre ersten Rückenschmerzen begonnen?

$I_{\text {Monat }}\left|I_{\ldots}\left\|_{\text {Jahr }}\right\|_{\ldots}\right|$

Strahlen die Schmerzen im Rückenbereich bis in die Beine aus? Ja $\square \quad$ Nein $\square$

Treten Ihre Schmerzen auch in der Nacht auf?

Ja $\square \quad$ Nein $\square$

....wenn ja: Ist Ihr Schlaf durch die Schmerzen beeinträchtigt?

Ja, meist $\square \quad$ Ja, oft $\square \quad$ Ja, sehr selten $\square \quad$ Nein $\square$ 
Bitte markieren Sie an dem Bild, wo sich Ihre schmerzhaften Stellen befinden: Falls Sie mehrere schmerzhafte Stellen haben, tragen Sie diese bitte geordnet nach Intensität ein

S1 = stärkster Schmerz; S2 = nächststärkster Schmerz...
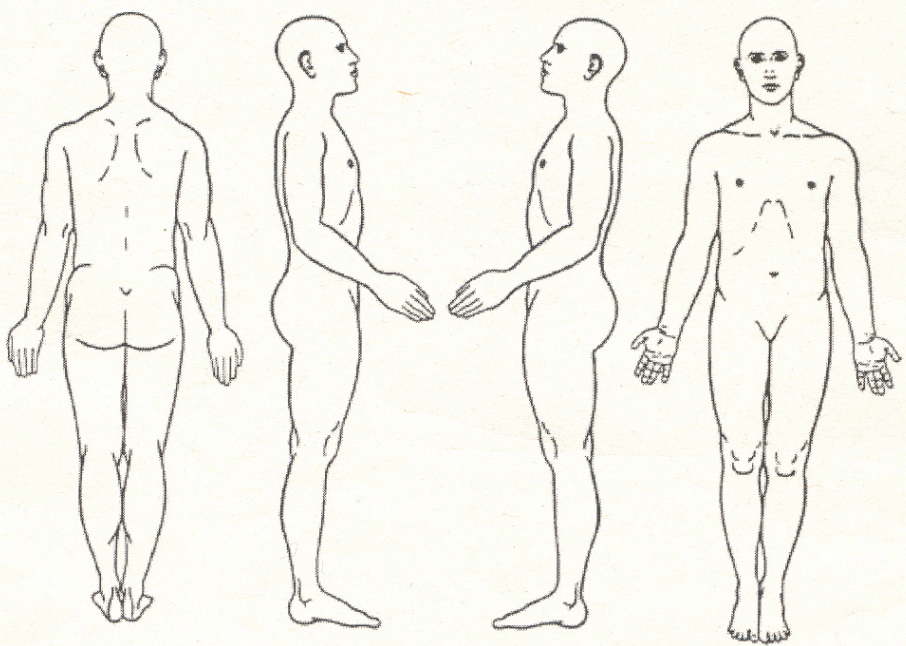

Bitte geben Sie im Folgenden die Stärke Ihrer Rückenschmerzen an. Kreuzen Sie auf den unten aufgeführten Linien an, wie stark Sie Ihre Schmerzen empfinden. Die Zahlen können Ihnen bei der Einteilung helfen: Ein Wert von 0 bedeutet, Sie haben keine Schmerzen, ein Wert von 10 bedeutet, Sie leiden unter Schmerzen, wie sie für Sie nicht stärker vorstellbar sind. Die Zahlen dazwischen geben Abstufungen der Schmerzstärke an.

a) Geben Sie bitte zunächst Ihre momentane Schmerzstärke an:

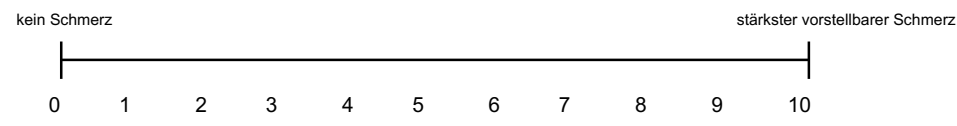

b) Geben Sie bitte Ihre durchschnittliche Schmerzstärke während der letzten 3 Monate an:

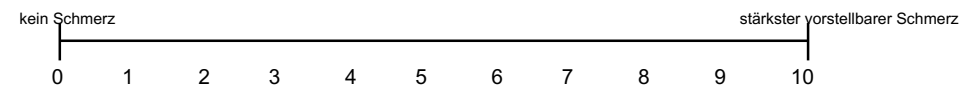

c) Geben Sie jetzt bitte Ihre größte Schmerzstärke während der letzten 3 Monate an:

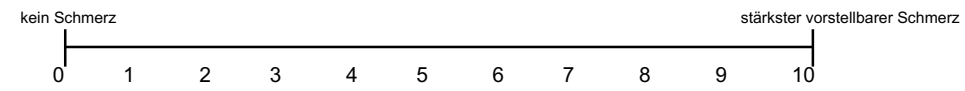

d) Geben Sie jetzt bitte Ihre geringste Schmerzstärke während der letzten 3 Monate an:

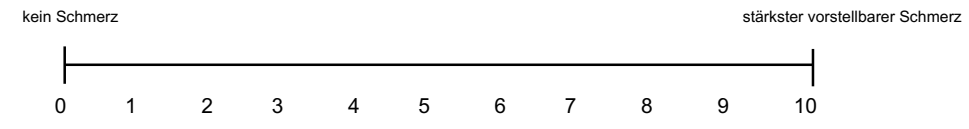


An ungefähr wie vielen Tagen konnten Sie in den letzten 3 Monaten aufgrund lhrer Rückenschmerzen Ihren üblichen Beschäftigungen (Beruf, Schule/Studium, Hausarbeit) nicht nachgehen? Tage

In den folgenden Fragen geht es um die Beeinträchtigung von Aktivitäten durch Rückenschmerzen. Sie können Ihre Angaben jeweils auf einer Skala von 0 bis 10 abstufen. Der Wert 0 bedeutet keine Beeinträchtigung, der Wert 10 bedeutet, dass Sie außerstande sind/waren, irgendetwas zu tun. Mit den dazwischen liegenden Werten können Sie Abstufungen vornehmen.

In welchem Maße haben die Schmerzen in den letzten drei Monaten Ihren Alltag (Ankleiden, Waschen, Essen, Einkaufen etc.) beeinträchtigt?

$$
\begin{array}{c|ccccccccc}
{\left[\begin{array}{l}
0] \\
\text { Keine } \\
\text { Beeinträchtigung }
\end{array}[1]\right.} & {[2]} & {[3]} & {[4]} & {[5]} & {[6]} & {[7]} & {[8]} & {[9]} & {\left[\begin{array}{c}
\text { [ 10 ] } \\
\text { Ich war außer Stande, } \\
\text { etwas zu tun }
\end{array}\right.}
\end{array}
$$

In welchem Maße haben die Schmerzen in den letzten drei Monaten Ihre Freizeitaktivitäten oder Unternehmungen im Familien- oder Freundeskreis beeinträchtigt?
[0]
[1] [2]
[3]
[ 4 ]
[ 5]
[ 6 ]
[ 7 ]
[ 8 ]
[9] [ 10 ]
Beeinträchtigung Ich war außer Stande, etwas zu tun

In welchem Maße haben die Schmerzen in den letzten drei Monaten Ihre Arbeitsfähigkeit (einschließlich Hausarbeit) beeinträchtigt?
[0]
[1] [2]
[3]
[ 4]
[ 5]
[6]
[ 7 ]
[ 8 ]
[ 9 ]
Beeinträchtigung

Von wem wurden Sie bisher wegen Ihrer Rückenschmerzen untersucht oder behandelt?

Keine Behandlung
Allgemeinarzt

Chirurg

Heilpraktiker

Internist

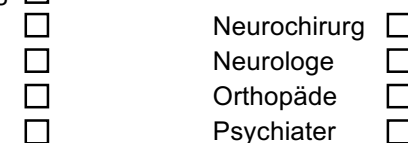

\section{Psychotherapeut \\ Radiologe \\ Schmerztherapeut}

Andere:

Wie oft sind Sie insgesamt aufgrund der Rückenschmerzen beim Arzt gewesen? I__l_ mal .

Wie oft sind Sie insgesamt aufgrund der Rückenschmerzen bei Therapeuten gewesen? _____ mal

Sind Sie in den letzten 12 Monaten aufgrund der Rückenschmerzen beim Arzt gewesen?
$\square$ nein
ja, I__l_ mal

Sind Sie in den letzten 12 Monaten aufgrund der Rückenschmerzen bei Therapeuten gewesen?
$\square$ nein
ja, |__l_ mal 
Wie wurden Ihre Rückenschmerzen bisher behandelt? Kreuzen Sie bitte an, welche der unten aufgeführten Behandlungsmaßnahmen Sie erhalten haben und wie oft.

Geben Sie bitte auch an, ob Ihre Schmerzen durch diese Maßnahmen zumindest zeitweise gelindert wurden.

\begin{tabular}{|c|c|c|c|c|c|}
\hline \multirow[b]{2}{*}{ bisher keine Schmerzbehandlung } & Erhalten: & Wenn ja $\rightarrow$ & \multicolumn{3}{|c|}{ wirksam? } \\
\hline & $\square$ & & ja & $\begin{array}{l}\text { vorüber- } \\
\text { gehend }\end{array}$ & nein \\
\hline Medikamente & $\square$ & $\rightarrow$ & $\square$ & $\square$ & $\square$ \\
\hline Infusionen & $\square$ & $\rightarrow$ & $\square$ & $\square$ & $\square$ \\
\hline Einspritzungen in das Schmerzgebiet, Nervenblockaden & $\square$ & $\rightarrow$ & $\square$ & $\square$ & $\square$ \\
\hline Einspritzungen am Rückenmark (z.B. epidural) & $\square$ & $\rightarrow$ & $\square$ & $\square$ & $\square$ \\
\hline Rückenmarksnahe Sonden- (SCS) oder Pumpensysteme & $\square$ & $\rightarrow$ & $\square$ & $\square$ & $\square$ \\
\hline Krankengymnastik & $\square$ & $\rightarrow$ & $\square$ & $\square$ & $\square$ \\
\hline Massagen, Bäder, Kälte-/Wärmetherapie & $\square$ & $\rightarrow$ & $\square$ & $\square$ & $\square$ \\
\hline Elektrische Nervenstimulation (TENS) & $\square$ & $\rightarrow$ & $\square$ & $\square$ & $\square$ \\
\hline Akupunktur & $\square$ & $\rightarrow$ & $\square$ & $\square$ & $\square$ \\
\hline Chiropraktik & $\square$ & $\rightarrow$ & $\square$ & $\square$ & $\square$ \\
\hline Psychotherapie & $\square$ & $\rightarrow$ & $\square$ & $\square$ & $\square$ \\
\hline Entspannungsverfahren, Hypnose, Biofeedback & $\square$ & $\rightarrow$ & $\square$ & $\square$ & $\square$ \\
\hline Operationen am Rücken & $\square$ & $\rightarrow$ & $\square$ & $\square$ & $\square$ \\
\hline Kur-/Reha-Behandlung & $\square$ & $\rightarrow$ & $\square$ & $\square$ & $\square$ \\
\hline Anderes: & $\square$ & $\rightarrow$ & $\square$ & $\square$ & $\square$ \\
\hline
\end{tabular}

Was tun Sie selbst, um Ihre Rückenschmerzen günstig zu beeinflussen?

Bitte machen Sie genaue Angaben, z.B. spazieren gehen, schlafen, Ablenkung, ...

Ich kann meine Rückenschmerzen nicht beeinflussen

Wurde bei Ihnen eine der folgenden Erkrankungen diagnostiziert? Zutreffendes bitte ankreuzen:

$\square$ Osteoporose

$\square \quad$ Rheumatische Erkrankungen im Rückenbereich

Bandscheibenvorfall

Nehmen Sie derzeit Schmerzmedikamente ein? Welche

$\square$ Nein $\square$ Ja $\square$ nicht täglich
ca. I_ll_ mal pro Woche
ca. I____ mal pro Monat

$\square$ täglich 


\section{Fragebogen IIP-32}

In unserem Leben haben wir tagtäglich viele soziale Kontakte. In Ihrem Berufsfeld kommen dazu noch die Kontakte mit Gästen oder auch Kollegen und Vorgesetzten hinzu. Im menschlichen Miteinander ist immer wieder viel kommunikativer Austausch nötig. Das kann mal belastend, aber auch erfreulich sein. Die folgenden Fragen beziehen sich generell auf Ihr Empfinden in sozialen Situationen.

\section{Anleitung}

Nachfolgend finden Sie eine Liste von Schwierigkeiten, die im Zusammenhang mit Beziehungen zu anderen berichtet werden. Bitte lesen Sie die Liste durch und überlegen Sie, ob die einzelnen Schwierigkeiten für Sie ein Problem darstellten und zwar bezogen auf irgendeine Person, die in Ihrem Leben eine bedeutsame Rolle spielte. Kreuzen Sie bitte für jedes Problem die Ziffer an, die beschreibt, wie sehr Sie darunter gelitten haben.

\section{Beispiel}

Wie sehr haben Sie unter diesem Problem gelitten?

Es fällt mir schwer...

0. mit meinen Angehörigen nicht wenig mittelmäßig ziemlich sehr

zurecht zu kommen

$\square \quad \square \quad \square \quad \square$

Teil I. Die nachstehenden Aspekte können im Umgang mit anderen Menschen schwierig sein.

Es fällt mir schwer...

\begin{tabular}{|c|c|c|c|c|c|}
\hline & nicht & wenig & $\begin{array}{l}\text { mittel- } \\
\text { mäßig }\end{array}$ & $\begin{array}{c}\text { ziem- } \\
\text { lich }\end{array}$ & sehr \\
\hline 1. anderen gegenüber „Nein“ zu sagen. & $\square$ & $\square$ & $\square$ & $\square$ & $\square$ \\
\hline 2. mich Gruppen anzuschließen. & 口 & $\square$ & $\square$ & $\square$ & $\square$ \\
\hline 3. mich fremden Menschen vorzustellen. & $\square$ & $\square$ & $\square$ & $\square$ & $\square$ \\
\hline 4. andere mit anstehenden Problemen zu konfrontieren. & 口 & 口 & $\square$ & $\square$ & $\square$ \\
\hline 5. mich gegenüber jemand anderem zu behaupten. & 口 & $\square$ & 口 & $\square$ & $\square$ \\
\hline 6. mich mit jemand anderem zu streiten. & $\square$ & $\square$ & $\square$ & $\square$ & $\square$ \\
\hline $\begin{array}{l}\text { 7. anderen gegenüber aggressiv zu sein, wenn es die Lage } \\
\text { erfordert. }\end{array}$ & 口 & 口 & 口 & 口 & 口 \\
\hline 8. anderen Menschen meine Zuneigung zu zeigen. & $\square$ & $\square$ & $\square$ & $\square$ & $\square$ \\
\hline 9. mit anderen zu recht zu kommen. & $\square$ & $\square$ & 口 & a & $\square$ \\
\hline $\begin{array}{l}\text { 10. meine Gefühle anderen gegenüber frei heraus zu } \\
\text { äußern. }\end{array}$ & $\square$ & $\square$ & $\square$ & 口 & 口 \\
\hline 11. wenn nötig, standfest zu sein. & a & $\square$ & 口 & $\square$ & $\square$ \\
\hline
\end{tabular}

Es fällt mir schwer... 


\begin{tabular}{|c|c|c|c|c|c|}
\hline & nicht & wenig & $\begin{array}{l}\text { mittel- } \\
\text { mäßig }\end{array}$ & $\begin{array}{l}\text { ziem- } \\
\text { lich }\end{array}$ & sehr \\
\hline 12. ein Gefühl von Liebe für jemanden zu empfinden. & $\square$ & $\square$ & $\square$ & $\square$ & 口 \\
\hline 13. mich anderen nahe zu fühlen. & 口 & $\square$ & 口 & ( & 口 \\
\hline 14. mich wirklich um die Probleme anderer zu kümmern. & 口 & 口 & ב & (a & 口 \\
\hline $\begin{array}{l}\text { 15. die Bedürfnisse eines anderen über meine eigenen zu } \\
\text { stellen. }\end{array}$ & a & a & 口 & 口 & $\square$ \\
\hline 16. mich aus den Angelegenheiten anderer herauszuhalten. & ב & a & a & 口 & 口 \\
\hline $\begin{array}{l}\text { 17. mich über das Glück eines anderen Menschen zu } \\
\text { freuen. }\end{array}$ & a & a & ב & a & 口 \\
\hline 18. andere zu bitten, mit mir etwas zu unternehmen. & 口 & 口 & ב & $\square$ & 口 \\
\hline $\begin{array}{l}\text { 19. fest und bestimmt zu bleiben, ohne mich darum zu } \\
\text { kümmern, ob ich die Gefühle anderer verletze. }\end{array}$ & a & a & $\square$ & ב & $\square$ \\
\hline
\end{tabular}

Teil II. Die nachstehenden Aspekte kann man im Übermaß tun.

\begin{tabular}{|c|c|c|c|c|c|}
\hline & nicht & wenig & $\begin{array}{l}\text { mittel- } \\
\text { mäßig }\end{array}$ & $\begin{array}{l}\text { ziem- } \\
\text { lich }\end{array}$ & sehr \\
\hline 20. Ich öffne mich anderen zu sehr. & $\square$ & $\square$ & $\square$ & $\square$ & $\square$ \\
\hline 21. Ich bin gegenüber anderen zu aggressiv. & $\square$ & $\square$ & $\square$ & $\square$ & $\square$ \\
\hline $\begin{array}{l}\text { 22. Ich lege zu viel Wert darauf, von anderen beachtet zu } \\
\text { werden. }\end{array}$ & $\square$ & $\square$ & $\square$ & $\square$ & $\square$ \\
\hline 23. Ich vertraue anderen zu leicht. & $\square$ & $\square$ & $\square$ & $\square$ & $\square$ \\
\hline 24. Ich bin zu sehr darauf aus, andere zu kontrollieren. & $\square$ & $\square$ & $\square$ & $\square$ & $\square$ \\
\hline $\begin{array}{l}\text { 25. Ich stelle zu oft die Bedürfnisse anderer über meine } \\
\text { eigenen. }\end{array}$ & $\square$ & $\square$ & $\square$ & $\square$ & $\square$ \\
\hline 26. Ich bin anderen gegenüber zu großzügig. & $\square$ & $\square$ & $\square$ & $\square$ & 口 \\
\hline $\begin{array}{l}\text { 27. Ich beeinflusse andere zu sehr, um zu bekommen, was } \\
\text { ich will. }\end{array}$ & $\square$ & 口 & $\square$ & $\square$ & 口 \\
\hline 28. Ich erzähle anderen zu oft persönliche Dinge. & $\square$ & $\square$ & $\square$ & $\square$ & $\square$ \\
\hline 29. Ich streite zu oft mit anderen. & $\square$ & $\square$ & $\square$ & $\square$ & $\square$ \\
\hline 30. Ich lasse mich zu leicht von anderen überreden. & $\square$ & $\square$ & $\square$ & $\square$ & $\square$ \\
\hline 31. Ich möchte mich zu sehr an anderen rächen. & $\square$ & $\square$ & $\square$ & $\square$ & $\square$ \\
\hline 32. Die Not eines anderen Menschen berührt mich zu sehr. & $\square$ & $\square$ & $\square$ & $\square$ & 口 \\
\hline
\end{tabular}


Dieser Fragebogen enthält 21 Gruppen von Aussagen. Bitte lesen Sie jede Gruppe sorgfältig durch Suchen Sie dann die eine Aussage in jeder Gruppe heraus, die am besten beschreibt, wie Sie sich in dieser Woche einschließlich heute gefühlt haben und kreuzen Sie die dazugehörige Ziffer (0, 1, 2 oder 3) an. Falls mehrere Aussagen einer Gruppe gleichermaßen zutreffen, können Sie auch mehrere Ziffern markieren. Lesen Sie auf jeden Fall alle Aussagen in jeder Gruppe, bevor Sie Ihre Wahl treffen.

0 Ich bin nicht traurig.

1 Ich bin traurig.

2 Ich bin die ganze Zeit traurig und komme nicht davon los.

3 Ich bin so traurig oder unglücklich, dass ich es kaum noch ertrage.

0 Ich sehe nicht besonders mutlos in die Zukunft.

1 Ich sehe mutlos in die Zukunft.

2 Ich habe nichts, worauf ich mich freuen kann.

3 Ich habe das Gefühl, dass die Zukunft hoffnungslos ist, und dass die Situation nicht besser werden kann.

C

0 Ich fühle mich nicht als Versager.

1 Ich habe das Gefühl, öfter versagt zu haben als der Durchschnitt.

2 Wenn ich mein Leben überblicke, sehe ich bloss eine Menge Fehlschläge.

3 Ich habe das Gefühl, als Mensch ein völliger Versager zu sein.

0 Ich kann die Dinge genauso genießen wie früher

1 Ich kann die Dinge nicht mehr so genießen wie früher.

2 Ich kann aus nichts mehr eine echte Befriedigung ziehen

3 Ich bin mit allem unzufrieden oder gelangweilt.

0 Ich habe keine Schuldgefühle.

1 Ich habe häufig Schuldgefühle.

2 Ich habe fast immer Schuldgefühle.

3 Ich habe immer Schuldgefühle.
$\mathbf{F}$

0 Ich habe nicht das Gefühl, gestraft zu sein

1 Ich habe das Gefühl, vielleicht bestraft zu werden.

2 Ich erwarte, bestraft zu werden.

3 Ich habe das Gefühl, bestraft zu sein.

0 Ich bin nicht von mir enttäuscht.

1 Ich bin von mir enttäuscht.

2 Ich finde mich fürchterlich.

3 Ich hasse mich

$\mathbf{H}$

0 Ich habe nicht das Gefühl, schlechter zu sein als alle anderen.

1 Ich kritisiere mich wegen meiner Fehler und Schwächen.

2 Ich mache mir die ganze Zeit Vorwürfe wegen meiner Mängel.

3 Ich gebe mir für alles die Schuld, was schiefgeht.

I

0 Ich denke nicht daran, mir etwas anzutun.

1 Ich denke manchmal an Selbstmord, aber ich würde es nicht tun.

2 Ich möchte mich am liebsten umbringen.

3 Ich würde mich umbringen, wenn ich die Gelegenheit hätte.

$J$

0 Ich weine nicht öfter als früher.

1 Ich weine jetzt mehr als früher.

2 Ich weine jetzt die ganze Zeit.

3 Früher konnte ich weinen, aber jetzt kann ich es nicht mehr, obwohl ich es möchte. 


\section{K}

0 Ich bin nicht reizbarer als sonst.

1 Ich bin jetzt leichter verärgert oder gereizt als früher.

2 Ich fühle mich dauernd gereizt.

3 Die Dinge, die mich früher geärgert haben, berühren mich nicht mehr.

L

0 Ich habe nicht das Interesse an Menschen verloren.

1 Ich interssiere mich jetzt weniger für Menschen als früher.

2 Ich habe mein Interesse an anderen Menschen zum größten Teil verloren.

3 Ich habe mein ganzes Interesse an anderen Menschen verloren.

\section{M}

0 Ich bin so entschlussfreudig wie immer.

1 Ich schiebe Entscheidungen jetzt öfter als früher auf.

2 Es fällt mir jetzt schwerer als früher, Entscheidungen zu treffen.

3 Ich kann überhaupt keine Entscheidungen mehr treffen.

\section{N}

0 Ich habe nicht das Gefühl, schlechter auszusehen als früher.

1 Ich mache mir Sorgen, dass ich alt oder unattraktiv aussehe.

2 Ich habe das Gefühl, dass Veränderungen in meinem Aussehen eintreten, die mich hässlich machen.

3 Ich finde mich hässlich.

\section{0}

0 Ich kann so gut arbeiten wie früher.

1 Ich muss mir einen Ruck geben, bevor ich eine Tätigkeit in Angriff nehme.

2 Ich muss mich zu jeder Tätigkeit zwingen.

3 Ich bin unfähig zu arbeiten.

$\mathbf{P}$

0 Ich schlafe so gut wie sonst.

1 Ich schlafe nicht mehr so gut wie früher.

2 Ich wache 1 bis 2 Stunden früher auf als sonst und es fällt mir schwer, wieder einzuschlafen.

3 Ich wache mehrere Stunden früher auf als sonst und kann nicht mehr einschlafen.
0 Ich ermüde nicht stärker als sonst.

1 Ich ermüde schneller als früher.

2 Fast alles ermüdet mich.

3 Ich bin zu müde, um etwas zu tun.

\section{$\mathbf{R}$}

0 Mein Appetit ist nicht schlechter als sonst.

1 Mein Appetit ist nicht mehr so gut wie früher

2 Mein Appetit hat sehr stark nachgelassen.

3 Ich habe überhaupt keinen Appetit mehr.

\section{S}

0 Ich habe in letzter Zeit kaum abgenommen.

1 Ich habe mehr als 2 Kilo abgenommen.

2 Ich habe mehr als 5 Kilo abgenommen.

3 Ich habe mehr als 8 Kilo abgenommen.

Ich esse absichtlich weniger, um abzunehmen:

$$
\square \mathrm{Ja} \quad \square \text { Nein }
$$

\section{$T$}

0 Ich mache mir keine größeren Sorgen um meine Gesundheit als sonst.

1 Ich mache mir Sorgen über körperliche Probleme, wie Schmerzen, Magenbeschwerden oder Verstopfung.

2 Ich mache mir so große Sorgen über gesundheitliche Probleme, dass es mir schwer fällt, an etwas anderes zu denken.

3 Ich mache mir so große Sorgen über gesundheitliche Probleme, dass ich an nichts anderes mehr denken kann.

\section{U}

0 Ich habe in letzter Zeit keine Veränderung meines Interesses an Sex bemerkt.

1 Ich interessiere mich weniger für Sex als früher

2 Ich interessiere mich jetzt viel weniger für Sex.

3 Ich habe das Interesse an Sex völlig verloren. 


\section{HADS}

AD:Zur vollständigen Beurteilung ihrer Erkrankung bitten wir Sie nun um einige persönliche Angaben. Man weiß heute, dass körperliche Krankheit und seelisches Befinden oft eng zusammenhängen. Deshalb beziehen sich die Fragen ausdrücklich auf Ihre allgemeine und seelische Verfassung Wir bitten Sie, jede Frage zu beantworten, und zwar so, wie es für Sie persönlich in den letzten 14 Tagen (inklusive heute) am ehesten zutraf bzw. zutrifft. Machen Sie bitte ein Kreuz für jede Feststellung und lassen Sie bitte keine aus. Überlegen Sie nicht lange, sondern wählen Sie die Antwort aus, die Ihnen auf Anhieb am zutreffendsten erscheint.

\begin{tabular}{|c|c|}
\hline $\begin{array}{l}\text { Ich fühle mich angespannt oder überreizt } \\
\square \text { meistens } \\
\square \text { oft } \\
\square \text { von Zeit zu Zeit / gelegentlich } \\
\square \text { überhaupt nicht }\end{array}$ & $\begin{array}{l}\text { Ich fühle mich in meinen Aktivitäten gebremst } \\
\square \text { fast immer } \\
\square \text { sehr oft } \\
\square \text { manchmal } \\
\square \text { überhaupt nicht }\end{array}$ \\
\hline $\begin{array}{l}\text { Ich kann mich heute noch so freuen } \\
\text { wie früher } \\
\begin{array}{l}\square \text { ganz genau so } \\
\square \text { nicht ganz so sehr } \\
\square \text { nur noch ein wenig } \\
\square \text { kaum oder gar nicht }\end{array}\end{array}$ & $\begin{array}{l}\text { Ich habe manchmal ein ängstliches } \\
\text { Gefühl in der Magengegend } \\
\square \text { überhaupt nicht } \\
\square \text { gelegentlich } \\
\square \text { ziemlich oft } \\
\square \text { sehr oft }\end{array}$ \\
\hline $\begin{array}{l}\text { Mich überkommt eine ängstliche Vorahnung, } \\
\text { dass etwas Schreckliches passieren könnte } \\
\square \text { ja, sehr stark } \\
\square \text { ja, aber nicht allzu stark } \\
\square \text { etwas, aber es macht mir keine Sorgen } \\
\square \text { überhaupt nicht }\end{array}$ & $\begin{array}{l}\text { Ich habe das Interesse an meiner äußeren } \\
\text { Erscheinung verloren } \\
\square \text { ja, stimmt genau } \\
\square \text { ich kümmere mich nicht so sehr, wie ich sollte } \\
\square \text { möglicherweise kümmere ich mich zu wenig } \\
\square \text { ich kümmere mich so viel darum wie immer D }\end{array}$ \\
\hline 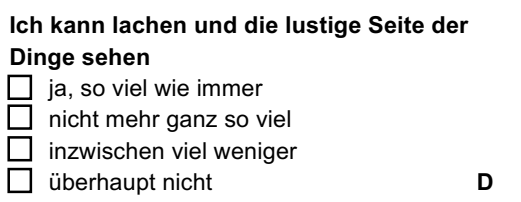 & $\begin{array}{l}\text { Ich fühle mich rastlos, muss immer in } \\
\text { Bewegung sein } \\
\begin{aligned} \square \text { ja, tatsächlich sehr } \\
\square \text { ziemlich } \\
\square \text { nicht sehr } \\
\square \text { überhaupt nicht }\end{aligned}\end{array}$ \\
\hline 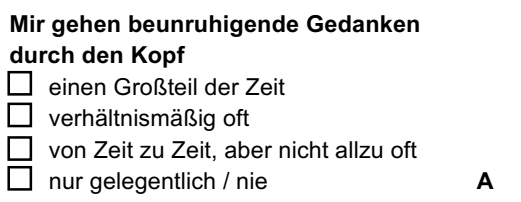 & $\begin{array}{l}\text { Ich blicke mit Freude in die Zukunft } \\
\square \text { ja, sehr } \\
\square \text { eher weniger als früher } \\
\square \text { viel weniger als früher } \\
\square \text { kaum bis gar nicht }\end{array}$ \\
\hline $\begin{array}{l}\text { Ich fühle mich glücklich } \\
\qquad \text { überhaupt nicht } \\
\square \text { selten } \\
\square \text { manchmal } \\
\square \text { meistens }\end{array}$ & $\begin{array}{l}\text { Mich überkommt plötzlich ein panikartiger } \\
\text { Zustand } \\
\square \text { ja, tatsächlich sehr oft } \\
\square \text { ziemlich oft } \\
\square \text { nicht sehr oft } \\
\square \text { überhaupt nicht }\end{array}$ \\
\hline 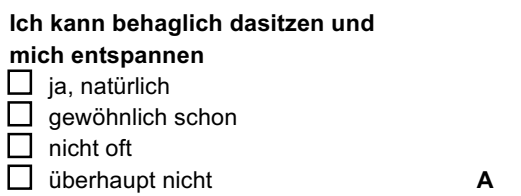 & $\begin{array}{l}\text { Ich kann mich an einem guten Buch, einer } \\
\text { Radio- oder Fernsehsendung erfreuen } \\
\begin{array}{l}\square \text { oft } \\
\square \text { manchmal } \\
\square \text { eher selten } \\
\square \text { sehr selten }\end{array}\end{array}$ \\
\hline
\end{tabular}




\section{Gesundheitszustand (SF-12)}

In diesem Fragebogen geht es um die Beurteilung Ihres allgemeinen Gesundheitszustandes. Ihre Antworten ermöglichen, im Zeitverlauf nachzuvollziehen, wie Sie sich fühlen und wie Sie im Alltag zurechtkommen.

Bitte beantworten Sie jede der folgenden Fragen, indem Sie bei den Antwortmöglichkeiten die Antwort ankreuzen, die am besten auf Sie zutrifft.

L-1. Wie würden Sie Ihren Gesundheitszustand im Allgemeinen beschreiben? (Bitte kreuzen Sie nur eine Antwort an!)

ausgezeichnet $\square \quad$ sehr gut $\square \quad$ gut $\square \quad$ weniger gut

schlecht

Im Folgenden sind einige Tätigkeiten beschrieben, die Sie vielleicht an einem normalen Tag ausüben. (Bitte kreuzen Sie in jeder Zeile nur eine Antwort an!)

\begin{tabular}{|l|l|c|c|c|}
\hline \multicolumn{2}{|l|}{$\begin{array}{l}\text { Sind Sie durch Ihren derzeitigen } \\
\text { Gesundheitszustand bei diesen Tätigkeiten } \\
\text { eingeschränkt? Wenn ja, wie stark? }\end{array}$} & $\begin{array}{c}\text { Ja, stark } \\
\text { eingeschränkt }\end{array}$ & $\begin{array}{c}\text { Na, etwas } \\
\text { eingeschränkt }\end{array}$ & $\begin{array}{c}\text { Nein, } \\
\text { überhaupt } \\
\text { nicht } \\
\text { eingeschränkt }\end{array}$ \\
\hline L-2. & $\begin{array}{l}\text { Mittelschwere Tätigkeiten, z.B. einen } \\
\text { Tisch verschieben, staubsaugen, kegeln, } \\
\text { Golf spielen }\end{array}$ & O & O & O \\
\hline L-3. & Mehrere Treppenabsätze steigen & 0 & 0 & 0 \\
\hline
\end{tabular}

Hatten Sie in den vergangenen 4 Wochen aufgrund Ihrer körperlichen Gesundheit irgendwelche Schwierigkeiten bei der Arbeit oder anderen alltäglichen Tätigkeiten im Beruf bzw. zu Hause?

\begin{tabular}{|l|l|l|l|}
\hline \multicolumn{2}{|l|}{ Schwierigkeiten } & Ja & Nein \\
\hline L-4. & Ich habe weniger geschafft als ich wollte & O & O \\
\hline L-5. & Ich konnte nur bestimmte Dinge tun & O & O \\
\hline
\end{tabular}

Hatten Sie in den vergangenen 4 Wochen aufgrund seelischer Probleme irgendwelche Schwierigkeiten bei der Arbeit oder anderen alltäglichen Tätigkeiten im Beruf bzw. zu Hause (z.B. weil Sie sich niedergeschlagen oder ängstlich fühlten)?

\begin{tabular}{|l|l|l|c|}
\hline \multicolumn{2}{|l|}{ Schwierigkeiten } & Ja & Nein \\
\hline L-6. & Ich habe weniger geschafft als ich wollte & O & O \\
\hline L-7. & Ich konnte nicht so sorgfältig wie üblich arbeiten & O & O \\
\hline \hline
\end{tabular}

L-8. Inwieweit haben die Schmerzen Sie in den vergangenen 4 Wochen bei der Ausübung Ihrer Alltagstätigkeiten zu Hause und im Beruf behindert?
überhaupt nicht $\square$
ein bisschen $\square$
Ziemlich $\square$
mäßig $\square$
sehr $\square$

In diesen Fragen geht es darum, wie Sie sich fühlen und wie es Ihnen in den vergangenen 4 Wochen gegangen ist. (Bitte kreuzen Sie in jeder Zeile nur eine Antwort an!)

\begin{tabular}{|c|c|c|c|c|c|c|c|}
\hline \multicolumn{2}{|c|}{$\begin{array}{l}\text { Wie oft waren sie in den } \\
\text { vergangenen } 4 \text { Wochen ... }\end{array}$} & immer & meistens & ziemlich oft & manchmal & selten & nie \\
\hline L-9. & ... ruhig und gelassen? & $\mathrm{O}$ & $\mathrm{O}$ & $\mathrm{O}$ & $\mathrm{O}$ & $\mathrm{O}$ & $\mathrm{O}$ \\
\hline L-10. & ... voller Energie? & $\mathrm{O}$ & $\mathrm{O}$ & $\mathrm{O}$ & $\mathrm{O}$ & $\mathrm{O}$ & $\mathrm{O}$ \\
\hline L-11. & ... entmutigt und traurig? & $\mathrm{O}$ & $\mathrm{O}$ & $\mathrm{O}$ & $\mathrm{O}$ & $\mathrm{O}$ & $\mathrm{O}$ \\
\hline
\end{tabular}

L-12. Wie häufig haben Ihre körperliche Gesundheit oder seelischen Probleme in den vergangenen 4 Wochen Ihre Kontakte zu anderen Menschen (Besuche bei Freunden, Verwandten usw.) beeinträchtigt?
immer $\square$
meistens $\square$
manchmal
selten $\square$
nie $\square$

Fragebogen zur Schmerzbewältigung: CSQ-D 
Erfahrungen haben gezeigt, dass Menschen, die unter Schmerzen leiden, unterschiedliche Wege entwickeln, um mit diesen Schmerzen umzugehen oder sie zu bewältigen. Das kann zum Beispiel bedeuten, dass Sie, wenn Sie Schmerzen haben, sich etwas zur Aufmunterung sagen oder sich mit einer Beschäftigung ablenken.

Im Folgenden finden Sie eine Liste verschiedener Tätigkeiten, die Menschen durchführen, wenn Sie schmerzen haben. Für jede Aktivität kreuzen Sie bitte an, wie oft Sie diese

durchführen, wenn Sie Schmerzen haben.

Eine 0 bedeutet: Ich mache das nie, wenn ich Schmerzen empfinde.

Eine 3 bedeutet: Ich mache das manchmal, wenn ich Schmerzen empfinde.

Eine 6 bedeutet: Ich mache das immer, wenn ich Schmerzen empfinde.

Wenn ich Schmerzen habe:

\begin{tabular}{|c|c|c|c|c|c|c|c|c|c|}
\hline \multirow{3}{*}{1.} & \multirow{3}{*}{$\begin{array}{l}\text { Ich versuche, Abstand von den Schmerzen zu gewinnen, } \\
\text { beinahe so, als wären die Schmerzen im Körper einer anderen } \\
\text { Person. }\end{array}$} & \multirow{2}{*}{\multicolumn{2}{|c|}{ nie }} & \multirow{2}{*}{\multicolumn{3}{|c|}{ manchmal }} & \multirow{2}{*}{\multicolumn{3}{|c|}{ immer }} \\
\hline & & & & & & & & & \\
\hline & & 0 & 1 & 2 & 3 & 4 & & & 6 \\
\hline 2. & $\begin{array}{l}\text { Ich gehe aus dem Haus und unternehme etwas, wie z.B. } \\
\text { einkaufen, spazieren gehen, ins Kino gehen. }\end{array}$ & 0 & 1 & 2 & 3 & 4 & 5 & & 6 \\
\hline 3. & Ich versuche, an etwas Angenehmes zu denken. & 0 & 1 & 2 & 3 & 4 & 5 & & 6 \\
\hline 4. & $\begin{array}{l}\text { Ich stelle mir den Schmerz als etwas anderes vor, z.B. als eine } \\
\text { dumpfe oder warme Empfindung. }\end{array}$ & 0 & 1 & 2 & 3 & 4 & 5 & & 6 \\
\hline 5. & $\begin{array}{l}\text { Es ist schrecklich für mich und ich habe das Gefühl, dass es nie } \\
\text { besser wird. }\end{array}$ & 0 & 1 & 2 & 3 & 4 & 5 & & 6 \\
\hline 6. & $\begin{array}{l}\text { Ich sage mir, dass ich tapfer sein und trotz der Schmerzen } \\
\text { weiter machen soll. }\end{array}$ & 0 & 1 & 2 & 3 & 4 & 5 & & 6 \\
\hline 7. & Ich lese, höre Musik, sehe fern oder Ähnliches. & 0 & 1 & 2 & 3 & 4 & 5 & & 6 \\
\hline 8. & $\begin{array}{l}\text { Ich spreche mir Mut zu und sage mir, dass ich mit den } \\
\text { Schmerzen fertig werden muss. }\end{array}$ & 0 & 1 & 2 & 3 & 4 & 5 & & 6 \\
\hline 9. & Ich nehme meine Medikamente ein. & 0 & 1 & 2 & 3 & 4 & 5 & & 6 \\
\hline 10. & $\begin{array}{l}\text { Ich zähle in Gedanken oder lasse mir eine Melodie durch den } \\
\text { Kopf gehen. }\end{array}$ & 0 & 1 & 2 & 3 & 4 & 5 & & 6 \\
\hline 11. & $\begin{array}{l}\text { Ich versuche mir die Schmerzen als anderes Gefühl } \\
\text { vorzustellen, z.B. als Taubheit. }\end{array}$ & 0 & 1 & 2 & 3 & 4 & & & 6 \\
\hline 12. & $\begin{array}{l}\text { Es ist furchtbar für mich und ich denke, die Schmerzen } \\
\text { überwältigen mich. }\end{array}$ & 0 & 1 & 2 & 3 & 4 & 5 & & 6 \\
\hline 13. & $\begin{array}{l}\text { Ich mache Gedankenspiele, um mich von den Schmerzen } \\
\text { abzulenken. }\end{array}$ & 0 & 1 & 2 & 3 & 4 & & & 6 \\
\hline 14. & $\begin{array}{l}\text { Ich habe das Gefühl, dass mein Leben nicht mehr lebenswert } \\
\text { ist. }\end{array}$ & 0 & 1 & 2 & 3 & 4 & & & 6 \\
\hline 15. & $\begin{array}{l}\text { Ich weiß, dass es irgendwann jemanden geben wird, der mir } \\
\text { hilft, so dass meine Schmerzen eine Zeit lang weggehen. }\end{array}$ & 0 & 1 & 2 & 3 & 4 & & & 6 \\
\hline 16. & $\begin{array}{l}\text { Ich betätige mich körperlich, gehe spazieren oder arbeite im } \\
\text { Garten. }\end{array}$ & 0 & 1 & 2 & 3 & 4 & 5 & & 6 \\
\hline 17. & Ich bete zu Gott, dass der Schmerz nicht lange anhält. & 0 & 1 & 2 & 3 & 4 & & & 6 \\
\hline 18. & $\begin{array}{l}\text { Ich versuche mir vorzustellen, die Schmerzen wären nicht } \\
\text { Teil meines Körpers, sondern etwas, was nicht zu mir gehört. }\end{array}$ & 0 & 1 & 2 & 3 & 4 & & & 6 \\
\hline 19. & Ich ruhe mich aus. & 0 & 1 & 2 & 3 & 4 & & & 6 \\
\hline 20. & Ich höre auf, über die Schmerzen nachzudenken. & 0 & 1 & 2 & 3 & 4 & 5 & & 6 \\
\hline 21. & $\begin{array}{l}\text { Ich denke an die Zukunft und was sein wird, wenn ich keine } \\
\text { Schmerzen mehr habe. }\end{array}$ & 0 & 1 & 2 & 3 & 4 & & & 6 \\
\hline
\end{tabular}




\begin{tabular}{|c|c|c|c|c|c|c|c|c|}
\hline 22. & Ich sage mir, dass es nicht weh tut. & 0 & 1 & 2 & 3 & 4 & 5 & 6 \\
\hline 23. & $\begin{array}{l}\text { Ich sage mir, dass die Schmerzen mich nicht an meinen } \\
\text { Aufgaben hindern dürfen. }\end{array}$ & 0 & 1 & 2 & 3 & 4 & 5 & 6 \\
\hline 24. & Ich schenke meinen Schmerzen keine Aufmerksamkeit. & 0 & 1 & 2 & 3 & 4 & 5 & 6 \\
\hline 25. & $\begin{array}{l}\text { Ich habe Vertrauen zu den Ärzten, dass es irgendwann eine } \\
\text { wirksame Schmerzbehandlung geben wird. }\end{array}$ & 0 & 1 & 2 & 3 & 4 & 5 & 6 \\
\hline 26. & Egal wie schlimm die Schmerzen sind, ich werde damit fertig. & 0 & 1 & 2 & 3 & 4 & 5 & 6 \\
\hline 27. & Ich tue so, als ob der Schmerz nicht da wäre. & 0 & 1 & 2 & 3 & 4 & 5 & 6 \\
\hline 28. & $\begin{array}{l}\text { Ich mache mir ständig Gedanken darüber, ob es wohl jemals } \\
\text { aufhören wird. }\end{array}$ & 0 & 1 & 2 & 3 & 4 & 5 & 6 \\
\hline 29. & Ich lege mich hin. & 0 & 1 & 2 & 3 & 4 & 5 & 6 \\
\hline 30. & Ich erinnere mich an vergangene schöne Erlebnisse. & 0 & 1 & 2 & 3 & 4 & 5 & 6 \\
\hline 31. & Ich denke an Menschen, mit denen ich gerne zusammen bin. & 0 & 1 & 2 & 3 & 4 & 5 & 6 \\
\hline 32. & Ich bete, dass die Schmerzen aufhören mögen. & 0 & 1 & 2 & 3 & 4 & 5 & 6 \\
\hline 33. & Ich stelle mich unter die Dusche oder nehme ein Bad. & 0 & 1 & 2 & 3 & 4 & 5 & 6 \\
\hline 34. & $\begin{array}{l}\text { Ich stelle mir vor, die Schmerzen wären außerhalb meines } \\
\text { Körpers. }\end{array}$ & 0 & 1 & 2 & 3 & 4 & 5 & 6 \\
\hline 35. & Ich mache weiter, als ob die Schmerzen nicht wären. & 0 & 1 & 2 & 3 & 4 & 5 & 6 \\
\hline 36. & $\begin{array}{l}\text { Ich betrachte die Schmerzen als Herausforderung und lasse } \\
\text { nicht zu, dass sie mich behindern. }\end{array}$ & 0 & 1 & 2 & 3 & 4 & 5 & 6 \\
\hline 37. & Obwohl es weh tut, mache ich weiter. & 0 & 1 & 2 & 3 & 4 & 5 & 6 \\
\hline 38. & $\begin{array}{l}\text { Ich habe das Gefühl, dass ich den Schmerz nicht mehr } \\
\text { aushalten kann. }\end{array}$ & 0 & 1 & 2 & 3 & 4 & 5 & 6 \\
\hline 39. & Ich versuche, mit anderen Menschen zusammen zu sein. & 0 & 1 & 2 & 3 & 4 & 5 & 6 \\
\hline 40. & Ich ignoriere die Schmerzen. & 0 & 1 & 2 & 3 & 4 & 5 & 6 \\
\hline 41. & Ich vertraue auf meinen Glauben an Gott. & 0 & 1 & 2 & 3 & 4 & 5 & 6 \\
\hline 42. & Ich habe das Gefühl, dass ich nicht mehr weiter kann. & 0 & 1 & 2 & 3 & 4 & 5 & 6 \\
\hline 43. & Ich denke an Dinge, die mir Freude machen. & 0 & 1 & 2 & 3 & 4 & 5 & 6 \\
\hline 44. & Ich tue alles, um mich von den Schmerzen abzulenken. & 0 & 1 & 2 & 3 & 4 & 5 & 6 \\
\hline 45. & $\begin{array}{l}\text { Ich beschäftige mich mit angenehmen Dingen oder gönne mir } \\
\text { etwas Gutes. }\end{array}$ & 0 & 1 & 2 & 3 & 4 & 5 & 6 \\
\hline 46. & Ich tue so, als gehörten die Schmerzen nicht zu mir. & 0 & 1 & 2 & 3 & 4 & 5 & 6 \\
\hline 47. & $\begin{array}{l}\text { Ich versuche mich zu beschäftigen, z.B. im Haushalt oder im } \\
\text { Garten oder mit meinem Hobby. }\end{array}$ & 0 & 1 & 2 & 3 & 4 & 5 & 6 \\
\hline 48. & $\begin{array}{l}\text { Ich mache mir einen warmen (oder kalten) Umschlag, massiere } \\
\text { die schmerzende Stelle oder Ähnliches. }\end{array}$ & 0 & 1 & 2 & 3 & 4 & 5 & 6 \\
\hline & & & & & & & & \\
\hline \multirow{2}{*}{\multicolumn{2}{|c|}{$\begin{array}{l}\text { Wenn Sie an all das denken, was Sie tun, um mit Ihren Schmerzen } \\
\text { fertig zu werden, wie viel Kontrolle haben Sie dann im Durchschnitt } \\
\text { über die Schmerzen? }\end{array}$}} & 0 & 1 & 2 & 3 & 4 & 5 & 6 \\
\hline & & & & & & & & \\
\hline \multicolumn{2}{|r|}{$\begin{array}{l}\text { Wenn Sie an all das denken, was Sie tun, um mit Ihren Schmerzen } \\
\text { fertig zu werden, wie stark können Sie die Schmerzen verringern? }\end{array}$} & 0 & 1 & 2 & 3 & 4 & 5 & 6 \\
\hline
\end{tabular}




\section{FRAGEBOGEN ZU RÜCKENSCHMERZEN}

Wenn Sie Rückenschmerzen haben, sind alltägliche Dinge manchmal schwer zu erledigen.

Diese Liste enthält einige Sätze, die andere Menschen verwendet haben, um ihre Situation zu beschreiben, wenn sie Rückenschmerzen haben. Wenn Sie die Liste durchlesen, fällt Ihnen vielleicht der eine oder andere Satz auf, weil er Ihre Beschwerden von heute gut beschreibt. Wenn Sie die Liste lesen, denken Sie bitte daran, wie Ihre Situation heute ist. Wenn Sie einen Satz lesen, der Ihre Situation heute beschreibt, kreuzen Sie diesen Satz bitte an. Falls ein Satz Ihre Situation nicht beschreibt, kreuzen Sie diesen Satz nicht an und gehen zum nächsten. Bitte denken Sie daran, nur die Sätze anzukreuzen, von denen Sie sicher sind, dass sie Ihre Situation heute beschreiben.

R- 1. $\square$ Wegen meiner Rückenschmerzen bleibe ich den größten Teil des Tages zu Hause.

R- 2. $\square$ Ich wechsle häufig meine Körperhaltung, um meinen Rücken zu entlasten.

R- 3. $\square$ Ich gehe wegen meiner Rückenschmerzen langsamer als sonst.

R- 4. $\square$ Wegen meiner Rückenschmerzen erledige ich keine der Arbeiten, die ich sonst im Haushalt erledige.

R- 5. $\square$ Wegen meiner Rückenschmerzen halte ich mich beim Treppensteigen am Geländer fest.

R- 6. $\square$ Wegen meiner Rückenschmerzen lege ich mich häufiger als sonst zum Ausruhen hin.

R- 7. $\square$ Wegen meiner Rückenschmerzen muss ich mich an etwas abstützen, um aus einem Polstersessel hochzukommen.

R- 8. $\square$ Wegen meiner Rückenschmerzen bitte ich andere Menschen, etwas für mich zu erledigen.

R- 9. $\square$ Wegen meiner Rückenschmerzen brauche ich zum Ankleiden länger als sonst.

R-10. $\square$ Wegen meiner Rückenschmerzen achte ich darauf, nur kurze Zeit zu stehen.

R-11. $\square$ Wegen meiner Rückenschmerzen achte ich darauf, mich so wenig wie möglich zu bücken oder niederzuknien.

R-12. $\square$ Wegen meiner Rückenschmerzen fällt es mir schwer, von einem Stuhl aufzustehen.

R-13. $\square$ Ich leide den größten Teil des Tages/der Nacht unter Rückenschmerzen.

R-14. $\square$ Meine Rückenschmerzen erschweren mir das Umdrehen im Bett.

R-15. $\square$ Wegen meiner Rückenschmerzen ist mein Appetit nicht besonders gut.

R-16. $\square$ Wegen meiner Rückenschmerzen habe ich Probleme beim Anziehen von Socken (oder Strümpfen/Strumpfhosen).

R-17. $\square$ Wegen meiner Rückenschmerzen gehe ich nur kurze Strecken.

R-18. $\square$ Wegen meiner Rückenschmerzen schlafe ich weniger als sonst.

R-19. $\square$ Wegen meiner Rückenschmerzen brauche ich beim Ankleiden Hilfe.

R-20. $\square$ Wegen meiner Rückenschmerzen verbringe ich den größten Teil des Tages sitzend.

R-21. $\square$ Wegen meiner Rückenschmerzen versuche ich, schwere Arbeiten im Haushalt zu vermeiden.

R-22. $\square$ Wegen meiner Rückenschmerzen bin ich reizbarer und übellauniger als sonst.

R-23. $\square$ Wegen meiner Rückenschmerzen gehe ich Treppen langsamer hinauf als sonst.

R-24. $\square$ Wegen meiner Rückenschmerzen verbringe ich den größten Teil des Tages im Bett. 
Das Mainzer Stadiensystem der Schmerzchronifizierung Mainz Pain Staging System (MPSS)

H.U Gerbershagen und B. Nagel, DRK-Schmerz Zentrum Mainz

- Testanweisung -

\section{Achse I: Zeitliche Aspekte der Schmerzes}

Erläuterungen:

- Die Fragen beziehen sich auf den Zeitraum der letzten 4 Wochen vor der Erhebung

- Bei multilokulären Schmerzen beziehen sich die Fragen auf den Hauptschmerz des Patienten

\subsection{Auftretenshäufigkeit der Schmerzen}

Frage: Wie oft traten Ihre (Haupt-) Schmerzen in den letzten 4 Wochen im allgemeinen (durchschnittlich) auf ?

Codiere

1: Die Schmerzen treten nicht täglich auf; oder sie treten maximal einmal täglich auf, gehen aber wieder auf Null zurück; es gibt längere schmerzfreie Intervalle (hierbei wird der Schlaf nicht als schmerzfreies Intervall gewertet). Bestanden keine Schmerzen in den letzten 4 Wochen wird ebenfalls mit 1 codiert

2: Die Schmerzen treten mehrmals täglich auf, gehen aber wieder bis auf Null zurück, es gibt schmerzfreie Intervalle (hierbei wird der Schlaf nicht als schmerzfreies Intervall gewertet)

3: Die Schmerzen sind bis auf seltene schmerzfreie Momente und Schmerzfreiheit im Schlaf dauernd vorhanden, d.h. der Schmerz geht im allgemeinen nicht auf Null zurück

\subsection{Schmerzdauer}

Frage: Wie lange hielten Ihre (Haupt-) Schmerzen in den letzten 4 Wochen im allgemeinen an ?

\section{Codiere}

1: Die Schmerzen halten in der Regel bis zu mehreren Stunden oder kürzer an

2: Die Schmerzen halten meist mehrere Tage, höchstens bis zu einer Woche lang an

3: Die Schmerzen halten meist länger als eine Woche an oder sie sind dauerhaft vorhanden

\subsection{Wechsel der Schmerzintensität}

\subsubsection{Dauerschmerzen}

Frage: Zeigten Ihre Schmerzen in den letzten 4 Wochen im allgemeinen Schwankungen in der Stärke, d.h. wechselten die Schmerzen zwischen leichten, mäßigen und starken Schmerzen? 


\section{Codiere}

1: Schwankungen um zwei oder mehr Skalenwerte (NSS 0-10), zweimal oder öfter pro Woche

2: Schwankungen um zwei oder mehr Skalenwerte (NSS 0-10), seltener als zweimal pro Woche

3: Konstante Schmerzintensität

\subsubsection{Anfallsartige Schmerzen}

Frage: Traten Ihre Schmerzen in den letzten 4 Wochen in unterschiedlicher Stärke auf, d.h. gab es leichte, mäßig starke und starke Schmerzanfälle?

Codiere

1: Die einzelnen Schmerzanfälle waren zumeist (50\% der Anfälle oder mehr) von unterschiedlicher Stärke (mehr als 2 Skalenwerte Differenz (NSS 0-10))

2: Die einzelnen Schmerzanfälle waren nur zeitweise (unter $50 \%$ der Anfälle) von unterschiedlicher Stärke (mehr als 2 Skalenwerte Differenz (NSS 0-10))

3: Die einzelnen Schmerzanfälle hatten (nahezu) immer die gleiche Intensität

\section{Achse II: Räumliche Aspekte des Schmerzes, Lokularität der Schmerzen}

\section{Erläuterungen:}

- Die Fragen beziehen sich auf den Zeitraum der letzten 4 Wochen vor der Erhebung

- Nachfragen: Gibt es Haupt- und Nebenschmerzen ? Welche Schmerzen gehören für Sie zusammen?

- Kritische Punkte: Unterschiedliche Lokalisationen, die vom Patienten als zusammengehörender Schmerz verstanden werden, werden mit 1 codiert (z.B. Kreuz-, Beinschmerzen, die gleichzeitig auftreten und als zusammengehörend erlebt werden). Gleiche Lokalisationen, die mit unterschiedlichen Schmerzen besetzt sind, werden mindestens mit 2 codiert (z.B. Dauerkopfschmerzen vom Nacken ausgehend und zusätzlich einseitige attackenartige Kopfschmerzen anderen Charakters)

- Die Schmerzen müssen für den Patienten von Krankheitswert gewesen sein; sie waren mit einer relevanten Beeinträchtigung für den Patienten verbunden

Frage: An welchen Körperregionen litten Sie in den letzten 4 Wochen unter Schmerzen? Wieviele Schmerzbilder konnten Sie dabei unterscheiden ?

Codiere

1: Der Patient klagt über ein für ihn zusammengehörendes Schmerzbild (an einer oder verschiedenen Regionen des Körpers)

2: Der Patient klagt über zwei abgrenzbare Schmerzbilder, die an einer oder an mehreren Stellen des Körpers lokalisiert sein können

3: Der Patient klagt über mehr als zwei voneinander abgrenzbare Schmerzbilder, oder seine Schmerzen nehmen mindestens $50 \%$ oder mehr der Körperoberfläche ein 


\section{Achse III: Medikamenteneinnahmeverhalten}

\subsection{Medikamenteneinnahme}

\section{Erläuterungen:}

- $\quad$ Die Frage bezieht sich auf den Zeitraum der letzten 4 Wochen vor der Erhebung

- Schmerzbezogene Medikamente werden in folgende 3 Gruppen eingeteilt Gruppe I: Nicht-Opioid-Monoanalgetika (u.a. NSAR, Paracetamol, Metamizol) Gruppe II: schwach und stark wirksame Opioide

Gruppe III: Analgetika-Mischpräparate, Migränemittel, Muskelrelaxantien, Tranquilizer, Antidepressiva, Neuroleptika, Antiepileptika und Cortisonderivate, sofern sie zur Schmerztherapie eingesetzt wurden

Frage: Haben Sie während der letzten 4 Wochen Medikamente gegen Ihre Schmerzen eingenommen?

Codiere

1: Keine Medikamente, oder Einnahme an weniger als 15 Tagen pro Monat

2: Bis maximal zwei Medikamente der Gruppe I, an mehr als 15 Tagen pro Monat

3: Mehr als zwei Medikamente der Gruppe I an mindestens 15 Tagen im Monat; oder zumindest ein Medikament der Gruppen II oder III, an mindestens 15 Tagen pro Monat

\subsection{Medikamentenentzugsbehandlungen}

Erläuterungen:

- Die Frage bezieht sich auf die gesamte Lebenszeit für die in Frage 2 angegebenen Schmerzlokalisationen

- Ein Entzug (oder Dosisreduktion) wird hier nur bewertet, wenn er (sie) im Rahmen einer ambulanten oder stationären ärztlichen Behandlung stattgefunden hat.

- Eine selbständige Dosisreduktion muß mit behandlungsbedürftigen Entzugsymptomen verbunden gewesen sein

Frage: Wurde bei Ihnen jemals ein Medikament, das Sie wegen Ihrer Schmerzen einnahmen, entzogen oder versucht, die Dosis wesentlich zu reduzieren?

\section{Codiere}

1: Keine Entzugsbehandlung oder erhebliche Dosisreduktion in der gesamten Vorgeschichte

2: Einmalig eine Entzugsbehandlung oder erhebliche Dosisreduktion in der Vorgeschichte

3: Mehrfach Entzugsbehandlungen oder erhebliche Dosisreduktionen in der Vorgeschichte

\section{Achse IV: Inanspruchnahme des Gesundheitswesens}

\section{Erläuterung:}

- Die Fragen beziehen sich auf die gesamte Lebenszeit für die in Frage 2 angegebenen Schmerzlokalisationen 


\subsection{Hausarztwechsel}

Erläuterung:

- Es werden nur Wechsel des Hausarztes bzw. persönlichen Arztes wegen erfolgloser Schmerzbehandlung gewertet. Umzug, Schließen der Praxis etc. gelten nicht (Konsultationen verschiedener Fachärzte mit oder ohne Überweisung werden hier nicht "gezählt")

Frage: Haben Sie wegen nicht erfolgreicher Behandlungen Ihrer Schmerzen jemals Ihren Hausarzt bzw. Ihren persönlichen Arzt gewechselt ?

\section{Codiere}

1: Kein Wechsel des Hausarztes bzw. des persönlichen Arztes

2: Ein Wechsel des Hausarztes bzw. des persönlichen Arztes

3: Mehr als ein Wechsel des Hausarztes bzw. des persönlichen Arztes

\subsection{Schmerzbezogene Krankenhausaufenthalte}

\section{Erläuterung:}

- Es werden nur schmerzbezogene stationäre Aufenthalte gewertet. Kuren und Aufenthalte in Rehabilitationseinrichtungen siehe unter 4.4

Frage: Wurden Sie bereits einmal wegen Ihrer Schmerzen stationär in einem Krankenhaus behandelt?

Codiere

1: Keine oder nur eine stationäre schmerzbezogene Krankenhausbehandlung (Diagnostik und/oder Therapie)

2: Zwei bis drei stationäre schmerzbezogene Krankenhausaufenthalte

3: Mehr als drei stationäre schmerzbezogene Krankenhausaufenthalte

\subsection{Schmerzbezogene Operationen}

\section{Erläuterungen:}

- Es werden nur hauptsächlich aufgrund der Schmerzen durchgeführte Operationen gewertet

- Operative Eingriffe zur Therapie der Grunderkrankung oder anderer mit der Erkrankung verbundener Symptome werden nicht gewertet (z.B.: Bandscheibenoperation bei schwerer Parese; Versorgung von Frakturen nach Trauma; Adhäsiolyse bei Subileus etc.)

- Bei Kopf-Gesichtsschmerzen nach schmerzbezogenen Zahnextraktionen (eine Sitzung gilt als eine OP), Kieferoperationen und HNO-ärztlichen Operationen fragen

- Bei anderen Schmerzen nach endoskopischen Eingriffen (im Bauchraum, an den Gelenken) fragen

- Erfolgten Narbenkorrekturen ? (Narbenkorrekturen aus kosmetischen Gründen zählen nicht)

- Es werden alle ambulant und stationär durchgeführten Eingriffe gezählt

Frage: Wurden Sie jemals aufgrund Ihrer Schmerzen operiert ? 


\section{Codiere}

1: Keine oder eine schmerzbezogene Operation

2: Zwei bis drei schmerzbezogene Operationen

3: Mehr als drei schmerzbezogene Operationen

\subsection{Schmerzbezogene Kuren und Aufenthalte in Rehabilitationseinrichtungen}

Erläuterung:

- Es werden nur schmerzbezogene Kuren und stationäre Aufenthalte in Rehabilitationseinrichtungen gewertet

Frage: Waren Sie aufgrund Ihrer Schmerzen jemals zu einem Kuraufenthalt oder zu einer stationären Rehabilitationsmaßnahme?

Codiere

1: Keine schmerzbezogene Kur oder Rehabilitationsmaßnahme

2: Eine oder zwei schmerzbezogene Kuren oder Rehabilitationsmaßnahmen

3: Mehr als zwei schmerzbezogene Kuren oder Rehabilitationsmaßnahmen 


\section{Das Mainzer Stadienmodell der Schmerz-Chronifizierung (MPSS) Auswertungsformular}

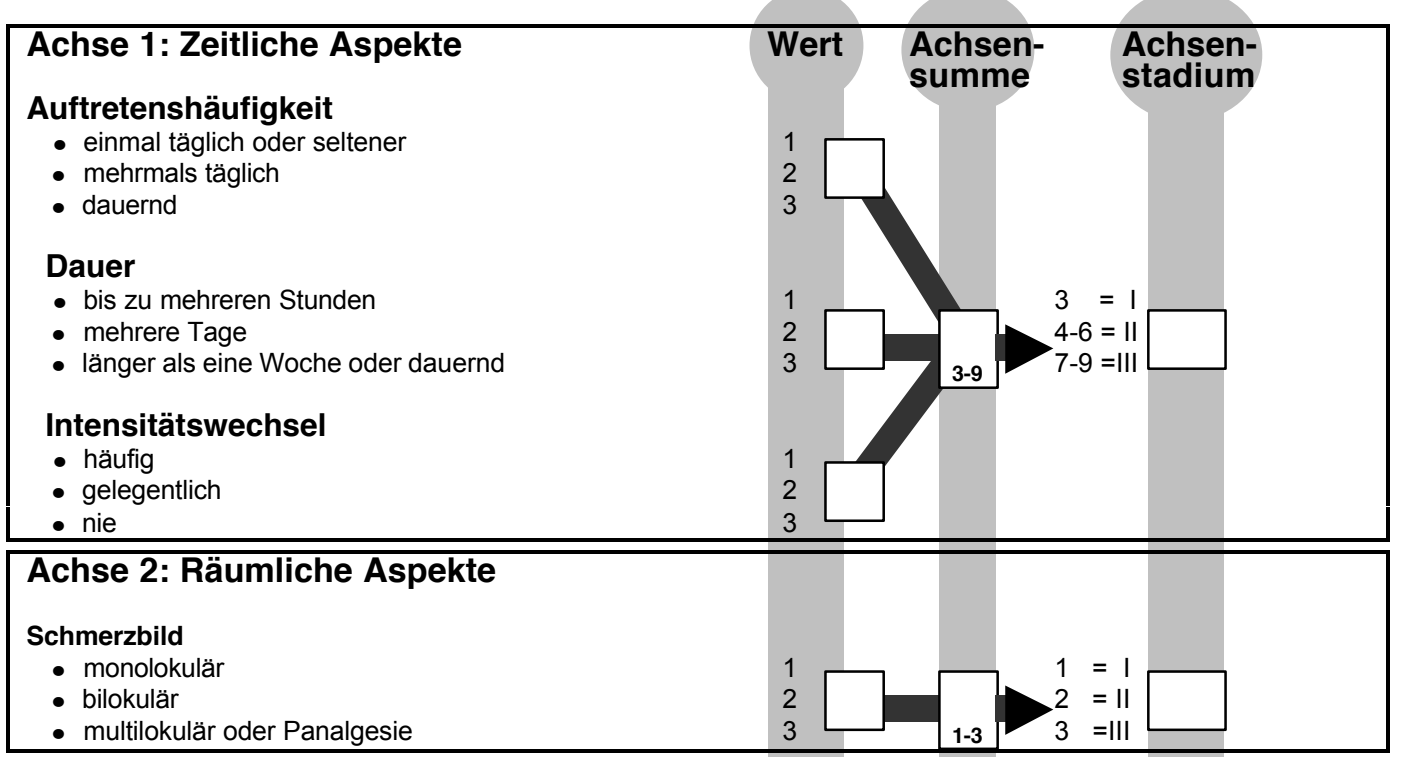

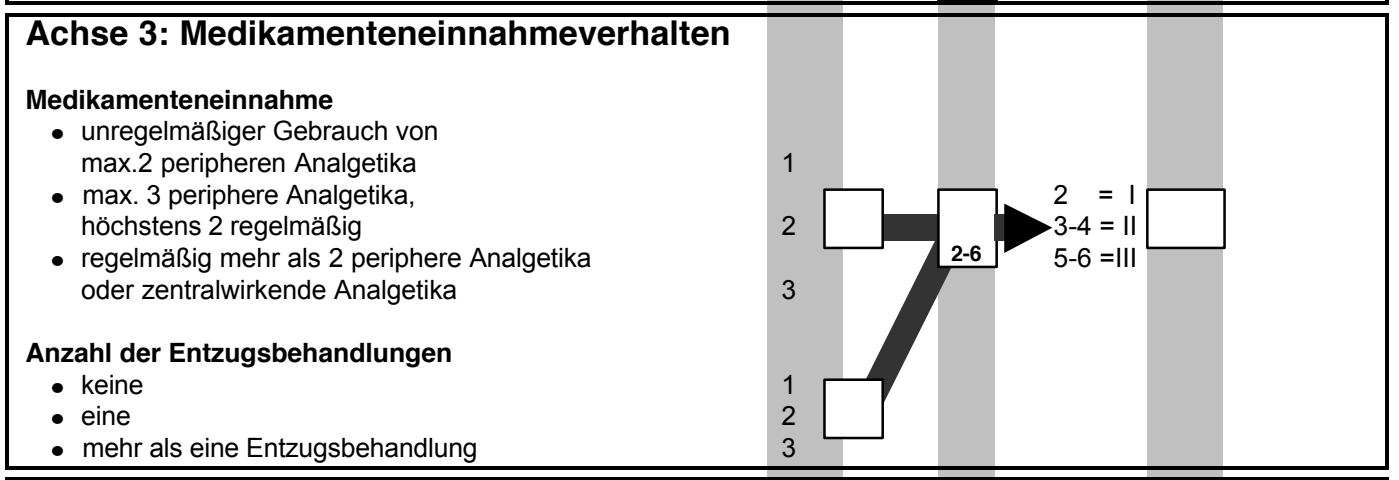

\begin{tabular}{|l|l|}
\hline Achse 4: Patientenkarriere \\
Wechsel des persönlichen Arztes \\
- kein Wechsel \\
- max 3 Wechsel \\
- mehr als 3 Wechsel \\
Schmerzbedingte Krankenhausaufenthalte \\
- bis 1
\end{tabular}

